\title{
ASIAN INTERNATIONAL STUDENTS' INTENTIONS TO SEEK COUNSELING: INTEGRATING COGNITIVE AND CULTURAL PREDICTORS
}

\author{
A Dissertation \\ Presented to \\ The Graduate Faculty of The University of Akron \\ In Partial Fulfillment \\ of the Requirements for the Degree \\ Doctor of Philosophy
}

Elena Sergeeva Yakunina

August, 2012 


\title{
ASIAN INTERNATIONAL STUDENTS' INTENTIONS TO SEEK COUNSELING: INTEGRATING COGNITIVE AND CULTURAL PREDICTORS
}

\author{
Elena Sergeeva Yakunina
}

\author{
Dissertation
}

Approved:

Advisor

Dr. Ingrid K. Weigold

Committee Member

Dr. Susan I. Hardin

Committee Member

Dr. John E. Queener

Committee Member

Dr. Robert C. Schwartz

Committee Member

Dr. Charles A. Waehler
Accepted:
Department Chair

Dr. Paul E. Levy

Dean of the College

Dr. Chand K. Midha

Dean of the Graduate School

Dr. George R. Newkome 


\begin{abstract}
Research has consistently identified low rates of counseling use among Asian international students. Empirical findings suggest that Asian foreign students are less likely to seek counseling than their American peers (Huyn, Quinn, Madon, \& Lustig, 2007; Nilsson, Berkel, Flores, \& Lucas, 2004; Yakushko, Davidson, \& Sanford-Martens, 2008). Research also indicates that when Asian international students do seek counseling, they typically attend few therapy sessions (Nilsson et al., 2004; Yakushko et al., 2008).

Several calls have been made to examine different cognitive and cultural predictors of counseling use in this population (Heggins \& Jackson, 2003; Ibrahim \& Ingram, 2007; Mori, 2000; Sheu \& Fukuyama, 2007). Possible cognitive predictors include help-seeking intentions, counseling attitudes, and stigma concerns. Potential cultural predictors include acculturation, Asian values, and loss of face concerns. The purpose of this study was to integrate the above predictors using the Theory of Reasoned Action (TRA, Ajzen \& Fishbein, 1980; Fishbein \& Ajzen, 2009).

In order to test the applicability of the TRA, data were collected from 295 Asian international students. All participants completed an online survey including a demographic questionnaire and measures of help-seeking intentions, counseling attitudes, stigma concerns, acculturation, Asian values, and loss of face concerns. Subsequently, three TRA models were tested: a Non-Mediated, a Partially Mediated, and a Fully Mediated Model (Holmbeck, 1997). Additional analyses included bivariate correlations and bootstrapping analysis of indirect effects (Shrout \& Bolger, 2002).
\end{abstract}


The results indicated partial support for the TRA as an integrated model of Asian international students' help-seeking. Path analysis indicated that the Partially Mediated Model offered the best data fit. In addition, participants' endorsement of Asian values was associated with more negative counseling attitudes, which then resulted into lower help-seeking intentions.

Contrary to the hypotheses, however, acculturation did not predict any of the helpseeking constructs of interest. In addition, participants' stigma and loss of face concerns were positively associated with their help-seeking intentions, which is inconsistent with prior theory and research. Overall, the findings of the present study indicate that Asian international students' help-seeking intentions may be more complex than what is represented by the TRA. 


\section{ACKNOWLEDGEMENTS}

When I look back on my journey of becoming a person and a counseling psychologist, I realize how much my path has been shaped by the important people in my life. The journey would have been lonely and aimless without your presence along the way.

My thanks go first and foremost to my mother, who taught me to be open and forthright, even if doing so meant taking a risk. To my father, I am grateful for giving me the courage to be better and to never settle down for less. From both of you I have inherited the persistence and tenacity that has kept me going forward. Thank you for giving me a beginning and then letting me choose a direction on my own.

My thanks go to Levius, who has been a partner, a brother, and a best friend throughout my years in college and graduate school. Thanks for being there even with the distance of a long distance relationship. You have given me strength and courage along the way.

I also owe thanks to my teachers and mentors, some of whom I may have forgotten, although I still remember the lessons they taught. I am thankful to the teachers who taught me how to read and write and how to love literature. Thanks go to my high school English teacher who helped me learn English and speak fluently in a language that was foreign to me. I owe thanks to my teachers in college who gave me guidance, advice, and recommendation letters. Without you, I would have probably never made it to graduate school. 
I want to thank my graduate academic advisor, Dr. Ingrid Weigold, for her patience, help, and advice in completing my dissertation. Thank you for staying excited, connected, and present throughout the ups-and-downs of my dissertation work. My thanks also go to the faculty, who provided both challenge and support throughout graduate school. You have taught me to question, reflect, and apply what I know in a way that has truly made me a scientist-practitioner. Thank you for helping me transform each challenge into an opportunity for growth. With your help, I was able to discover my strengths and develop as a counseling psychologist.

Thanks also go to my friends and classmates for sharing the journey and being a source of company, encouragement, and inspiration along the way. Wherever your careers may lead you, I believe that you will leave a mark. 


\section{TABLE OF CONTENTS}

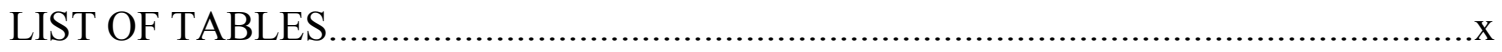

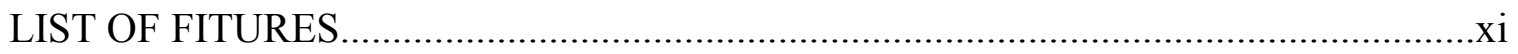

\section{CHAPTER}

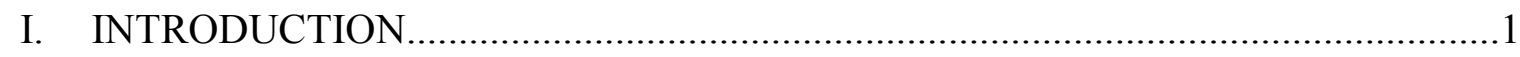

Asian International Students' Use of Counseling.......................................................1

The Need to Examine Cognitive Predictors....................................................................

The Need to Examine Cultural Predictors................................................................

Critical Issues in the Existing Literature...............................................................10

The Theory of Reasoned Action as a Model...............................................................13

The Purpose of the Present Study..............................................................................17

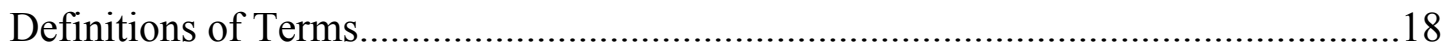

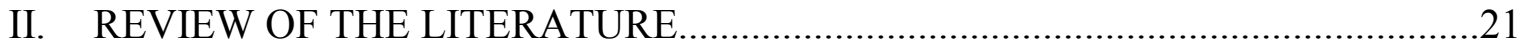

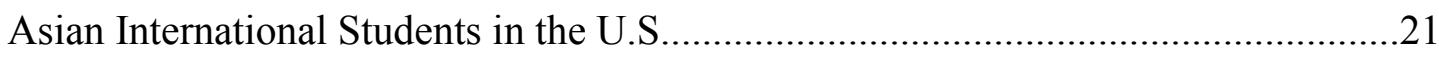

Asian International Students' Counseling Use..........................................................27

Intentions to Seek Psychological Counseling............................................................33

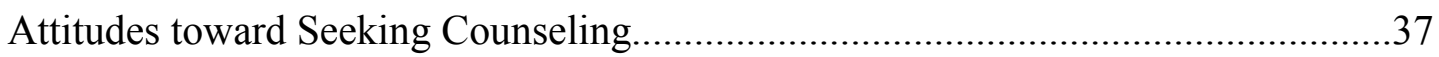

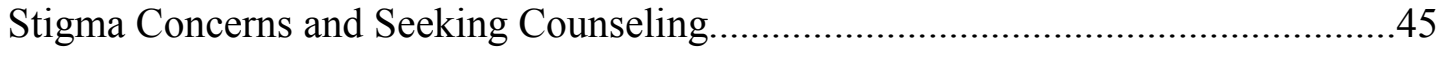

Acculturation and Seeking Counseling.................................................................50

Asian Values and Seeking Counseling.....................................................................61 
Loss of Face Concerns and Seeking Counseling.............................................. 71

The Theory of Reasoned Action as a Framework.................................................79

Summary, Theoretical Models, and Hypotheses................................................. 89

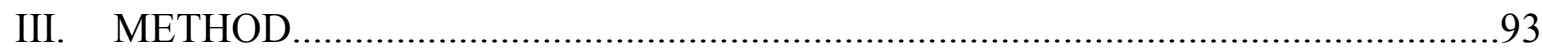

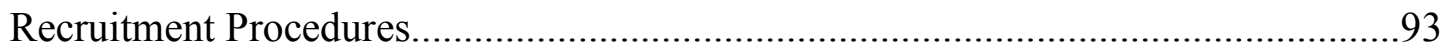

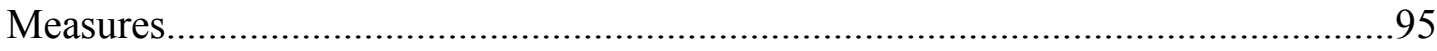

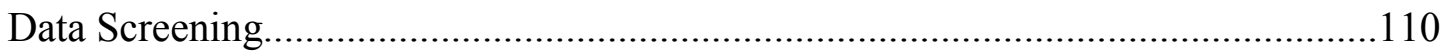

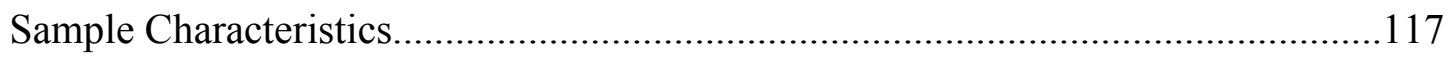

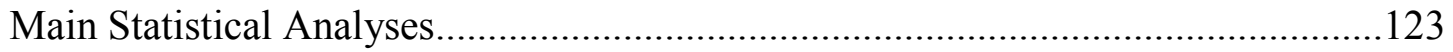

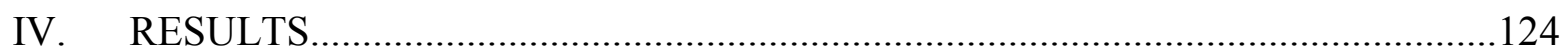

Determining the Adequacy of the Sample Size.................................................. 124

Testing the Hypothesized Bivariate Correlations..............................................124

Testing the Hypothesized TRA Models.......................................................... 128

Testing the Hypothesized Indirect Effects......................................................136

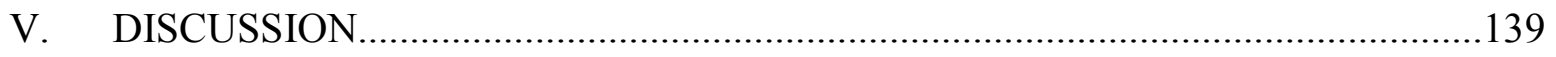

Summary of Hypotheses and Research Findings.............................................139

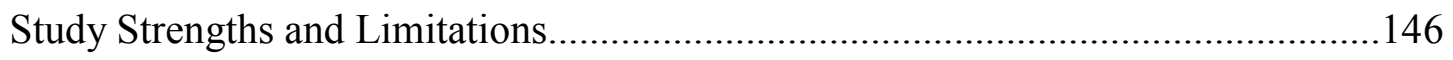

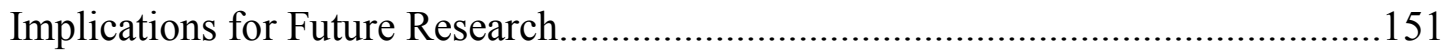

Implications for Clinical Practice ........................................................... 157

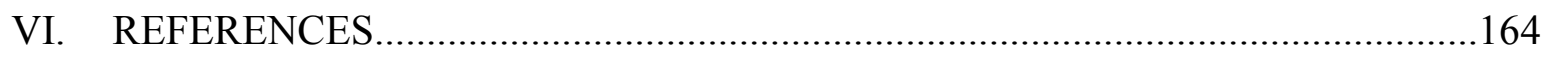

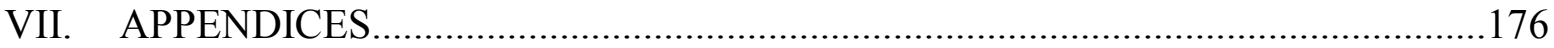

APPENDIX A. IRB LETTER OF APPROVAL............................................... 177

APPENDIX B. LEADING INSTITUTIONS HOSTING INTERNATIONAL

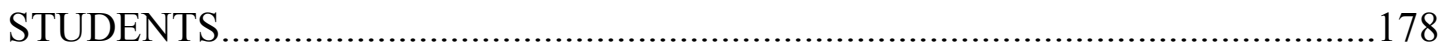


APPENDIX C. PARTICIPANT E-MAIL INVITATION........................................181

APPENDIX D. PARTICIPANT INFORMED CONSENT FORM..........................182

APPENDIX E. OPTIONAL RAFFLE ENTRY FORM..........................................184

APPENDIX F. DEMOGRAPHIC QUESTIONNAIRE..........................................185

APPENDIX G. INTENTIONS TO SEEK COUNSELING INVENTORYPSYCHOLOGICAL AND INTERPERSONAL CONCERNS...............................188

APPENDIX H. ATTITUDES TOWARD SEEKING PROFESSIONAL PSYCHOLOGICAL HELP SCALE - SHORT FORM............................................189

APPENDIX I. PERCEPTIONS OF STIGMATIZATION BY OTHERS FOR SEEKING PSYCHOLOGICAL HELP SCALE.....................................................191

APPENDIX J. ASIAN AMERICAN MULTIDIMENSIONAL ACCULTURATION SCALE - IDENTIFICATION WITH AMERICAN CULTURE

APPENDIX K. ASIAN AMERICAN VALUES SCALE -

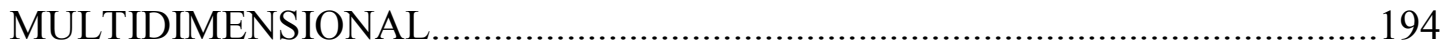

APPENDIX L. LOSS OF FACE QUESTIONNAIRE.............................................198 


\section{LIST OF TABLES}

Table

Page

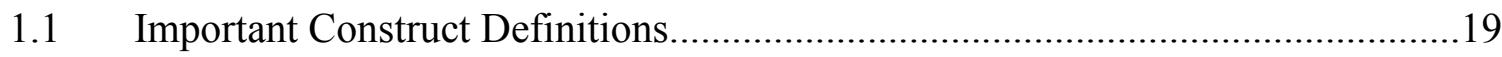

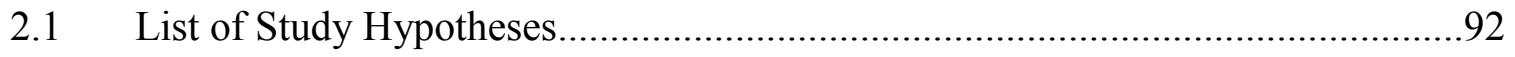

3.1 Chi Square Tests for Survey Completers and Non-Completers.......................114

3.2 Descriptive Statistics and Internal Consistencies.......................................116

3.3 Participant Age, Gender, Relationship Status, Academic Rank, and Field of

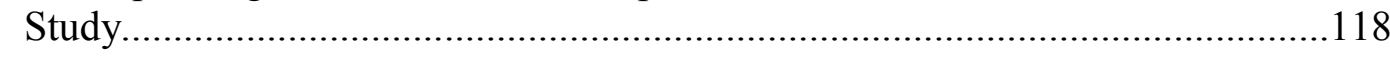

3.4 Participant Ethnicity, Nationality, Visa Type, and Years in the U.S..................119

3.5 Awareness of Mental Health Resources and Prior Use of Counseling...............120

3.6 Differences Between Participants With and Without Experience Seeing a Mental Health Professional................................................................................122

4.1 Bivariate Correlations Among the Constructs of Interest................................127

4.2 Comparisons of the Hypothesized TRA Models...........................................134

4.3 Bootstrap Analysis of Indirect Effects.................................................. 138

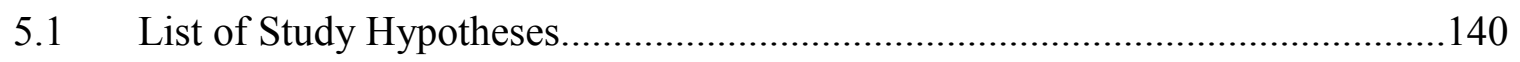




\section{LIST OF FIGURES}

Figure $\quad$ Page

1.1 Theory of Reasoned Action Model................................................................... 15

1.2 Theoretical Model Guiding the Present Study...............................................17

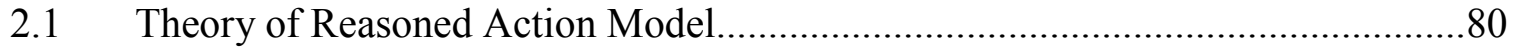

2.2 Model Integrating Cultural Predictors into the TRA.....................................91

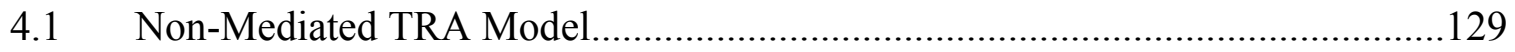

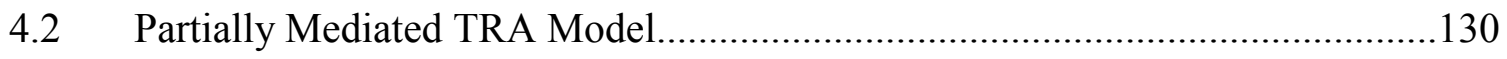

4.3 Fully Mediated TRA Model.....................................................................131 


\section{CHAPTER I}

\section{INTRODUCTION}

This chapter introduces the present study on Asian international students' intentions to seek counseling. I will first discuss several empirical findings that point to reduced use of counseling among Asian international students. I will then argue that these low rates of counseling use demands a look at several cognitive and cultural predictors. In particular, I will argue that cognitive factors, such as help-seeking intentions, counseling attitudes, and stigma concerns, may be important predictors of Asian international students' use of professional mental health services. Furthermore, I will assert that cultural factors, such as acculturation, Asian values, and loss of face concerns, also may be important factors impacting counseling service use in this population. Finally, I will highlight the need for a theory-driven model to guide future research on this topic. After examining several critical issues in the existing empirical literature, I will present a conceptual model of Asian international students' intentions to seek counseling that integrates both cognitive and cultural predictors and is grounded in the Theory of Reasoned Action (TRA, Ajzen \& Fishbein, 1980; Fishbein \& Ajzen, 2009). I will conclude this chapter by providing brief definitions of relevant terms.

\section{Asian International Students' Use of Counseling}

The existing empirical literature points to reduced use of counseling among international students in general, and Asian international students in particular. Research 
indicates, for example, that international students in general, and foreign-born Asian students specifically, are less likely to seek psychological counseling as compared to American students. Yakushko, Davidson, and Sanford-Martens (2008), for instance, found that only $1.8 \%$ of international students enrolled at a large, Midwestern university sought services at the local counseling center over a 5 -year period. Similarly, Nilsson, Berkel, Flores, and Lucas (2004) found that, although international students represented $8 \%$ of the student body, they constituted only $2.6 \%$ of the clients seen at the college counseling center over one academic year. Consistent with these results, Huyn, Quinn, Madon, and Lustig (2007) found that foreign graduate students were significantly less likely than American graduate students to have accessed college counseling in the past (17\% vs. $36 \%$, respectively) or to consider doing so in the future (33\% vs. $56 \%$, respectively). These authors also found that Asian international students in particular were significantly less likely to seek counseling than were foreign students from other geographic regions, such as Africa, South America, and Europe.

Additional empirical evidence also suggests that international students in general, and foreign-born Asian students specifically, may be significantly less likely to self-refer to counseling as compared to American students. In a study by Mitchell, Greenwood, and Gugliemi (2007), for example, international students who sought college counseling were significantly less likely to be self-referred ( $48 \%$ vs. $60 \%)$ and significantly more likely to be referred by faculty or staff ( $30 \%$ vs. $15 \%$ ) than were American students. Further research also suggests that Asian international students in particular may be significantly less likely to self-refer to counseling, as compared to foreign students coming from other geographic regions. One study found that international students from Asia were 
significantly less likely to self-refer to the college counseling center than were international students from Europe and Latin America (Yakushko et al., 2008). These findings indicate that foreign students as a group, and Asian international students specifically, may be hesitant to seek counseling and may do so only at the urging of concerned faculty, friends, or significant others.

Research also indicates that when international students do seek counseling, they frequently attend few sessions and terminate therapy prematurely. In a study of international students' counseling service use, for example, Anderson and Myer (1985) found that $33 \%$ of foreign-born students who sought therapy at a college counseling center failed to return for a second appointment, as compared to $12 \%$ of American students. In a similar study, Yakushko and colleagues (2008) observed that $36 \%$ of foreign students who sought college counseling terminated therapy after the first appointment, with a client no-show as the most common therapy termination. Available evidence also suggests that Asian international students in particular may be significantly more likely to terminate therapy prematurely, as compared to students from other regions. For example, Yakushko and colleagues (2008) found that Asian international students who sought college counseling attended significantly fewer sessions than did students coming from Central and South America. Similarly, Mitchell et al. (2007) observed that Asian international clients attended significantly fewer therapy sessions than did international students from Europe or North America.

Finally, research also suggests that international students in general may avoid seeking psychological help not just from mental health professionals, but also from informal support networks (e.g., friends, significant others, and extended family), leaving 
these students with few sources of help in the case of a psychological problem. In a study by Mau and Jepsen (1990), for example, international students were asked to rank-order their preferred sources of help for a psychological problem, with options including both formal (e.g., psychologist, psychiatrist, physician, nurse) and informal (e.g., friends, family, significant other, no-one) sources of help. International students in this study overwhelmingly selected "no-one" as their preferred source of help, indicating that these students may see psychological help-seeking as stigmatizing and inappropriate. Furthermore, Olivas and Li (2006) observed that, although international students indicated that they could easily seek emotional support from friends and family, many chose not to because of the fear of causing unnecessary worry for their significant others back home. Similarly, in a qualitative study of Asian international women's adjustment, Constantine, Kindaichi, Okazaki, Gainor, and Baden (2005) found that participants hesitated to share psychological problems with their families for fear of causing unnecessary worry and concern. Finally, international students may have more limited access to their informal support systems back home due to factors such as geographic distance, time zone differences, and financial and immigration problems that may impede frequent visits back home (Kaczmarek, Matlock, Merta, Ames, \& Ross, 1995). Given their limited access to informal support networks at home, many international students might benefit from the supportive services provided by college counseling.

The precise reasons for Asian international students' reduced use of counseling are not fully understood. In recent years several theoretical articles and book chapters have been published that attempt to explain the low use of counseling services by this population (Heggins \& Jackson, 2003; Ibrahim \& Ingram, 2007; Johnson \& Sandhu, 
2007; Mori, 2000; Sheu \& Fukuyama, 2007). Central in these theoretical writings has been the need to examine the interplay of cognitive variables (e.g., help-seeking intentions, counseling attitudes, and stigma concerns) as precursors of counseling utilization in this population (Heggins \& Jackson, 2003; Mori, 2000; Johnson \& Sandhu, 2007). Another central theme has been the need to examine a constellation of cultural variables (e.g., acculturation, Asian values, and loss of face concerns) that may account for Asian international students' reduced use of psychological services (Ibrahim \& Ingram, 2007; Johnson \& Sandhu, 2007; Sheu \& Fukuyama, 2007). Thus, it appears that future research should examine both cognitive and cultural predictors of Asian international students' counseling service utilization.

\section{The Need to Examine Cognitive Predictors}

One cognitive predictor that has been cited as central to understanding Asian international students' counseling use is help-seeking intentions. This construct is especially important to consider because preliminary findings point to reduced helpseeking intentions in this population. In a study by Mau and Jepsen (1990), for instance, Chinese international students were significantly less likely to seek professional counseling for a psychological problem, as compared to American students. Chinese students in this sample chose "no-one" as their preferred source of help for psychological difficulties, in contrast to American students who favored a psychiatrist. Similarly, Brinson and Kottler (1995) observed that Asian international students held negative views about counseling and indicated low intentions to seek mental health services. Asian international students in this study saw counseling as shameful and socially stigmatized, leading to low intentions to seek psychological help. Finally, Constantine and colleagues 
(2005) found that Asian international college women considered counseling as their last option in dealing with the adjustment difficulties of studying abroad. Participants in this study indicated that they would avoid seeking counseling because of concerns with bringing shame and social stigma to their families. Thus, it appears that Asian international students may endorse low intentions to seek mental health services, leading to low rates of counseling utilization.

Apart from help-seeking intentions, counseling attitudes also have been cited as an important predictor of mental health service use in this population. Preliminary evidence suggests that Asian international students hold negative attitudes toward seeking counseling. In an early study, Tedeschi and Willis (1993) found that Asian foreign students held significantly more negative counseling attitudes than did American students. Asian international students in this study expressed a lower perceived need for counseling, less emotional openness, and less confidence in the mental health field than did U.S.-born students. A similar study by Yoon and Jepsen (2008) indicated that Asian international students held more negative attitudes as expressed in less emotional openness, greater shame, and less self-perceived need for counseling than did U.S.-born students. Furthermore, Dadfar and Friedlander (1982) observed that Asian international students held more negative counseling attitudes than did international students from Europe and Latin America. In this study, Asian students expressed less confidence in mental health professionals and a greater preference for dealing with psychological problems on their own, as compared to students from other geographic areas. Thus, existing evidence suggests that foreign-born students from Asia may hold especially negative counseling attitudes, leading to lower rates of mental health service utilization. 
Apart from help-seeking intentions and counseling attitudes, stigma concerns also have been cited as a barrier to Asian international students' mental health service use. In a study by Tedeschi and Willis (1993) Asian international students expressed significantly more counseling stigma concerns and lower stigma tolerance, as compared to a matched sample of U.S.-born European American undergraduates. Similarly, Yoon and Jepsen (2008) found that, compared to U.S.-born students, Asian international students expressed greater shame and stigma concerns in the context of seeking psychological services. Furthermore, findings by Zhang and Dixon (2003) suggest that less acculturated Asian international students may experience greater stigma concerns. In this particular study, less acculturated Asian international students expressed greater fear of incurring social stigma, as well as lower tolerance toward public stigmatization. Finally, Dadfar and Friedlander (1982) observed that Asian foreign students who endorsed more stigma concerns were also significantly less likely to have used counseling in the past. This empirical literature suggests that counseling stigma may be a salient consideration for many Asian international students and, thus, may serve as a barrier to professional mental health service use in this population.

\section{The Need to Examine Cultural Predictors}

Apart from attending to cognitive predictors of counseling use, researchers also need to explore several cultural variables that may be relevant to an Asian international student population, such as acculturation, Asian values, and loss of face (Ibrahim \& Ingram, 2007; Johnson \& Sandhu, 2007; Sheu \& Fukuyama, 2007). Preliminary empirical evidence indicates, for example, that greater acculturation may facilitate psychological help-seeking among Asian American and Asian international populations. 
Using a sample of U.S.-born Asian American undergraduates, for instance, Atkinson and Gim (1989) found that more highly acculturated participants endorsed more positive counseling attitudes, as expressed in greater emotional openness and a stronger selfperceived need for counseling. Similarly, Tata and Leung (1994) observed that more highly acculturated Chinese American students endorsed significantly more positive counseling attitudes. Furthermore, in a combined sample of U.S.-born Asian American and foreign-born Asian international students, Liao and colleagues (2005) found that more highly acculturated participants held more positive attitudes and expressed greater intentions to seek professional help for a psychological problem. Finally, building on prior research by examining Asian international students, Zhang and Dixon (2004) found that greater acculturation predicted more positive counseling attitudes in this population, with more highly acculturated students reporting greater confidence in the mental health field. Thus, empirical evidence suggests that acculturation may be an important predictor of Asian international students' counseling attitudes and mental health service utilization. Apart from acculturation, Asian values also have been referenced as an important predictor of Asian American and Asian international students' counseling service use. In a study by Kim (2007), Asian American undergraduates who endorsed the values of collectivism, emotional self-control, and personal humility expressed significantly less positive counseling attitudes. In another study, Gloria, Castellanos, Park, and Kim (2008) found that, among Korean American undergraduates, stronger adherence to traditional Asian values predicted less positive attitudes toward seeking counseling. Further research indicated that cultural values also may predict Asian American students' stigma concerns and intentions to seek professional psychological help in the future (Shea \& Yeh, 2008). 
These authors found, for example, that Asian American undergraduates who held more traditional Asian values also expressed greater stigma concerns and less positive attitudes in the context of seeking counseling. Similarly, Miville and Constantine (2007) found that Asian American college women who endorsed more traditional Asian values also expressed greater stigma concerns and lower intentions to seek mental health services in the future. Finally, in a study that combined U.S.-born Asian American and foreign-born Asian international students in a single sample, Liao, Rounds, and Klein (2005) found that greater endorsement of Asian values predicted less positive counseling attitudes, which, in turn, related to lower intentions to seek psychological services.

Apart from acculturation and Asian values, loss of face has also been referenced as an important predictor of counseling use among Asian American and Asian international populations. Using a predominantly Asian American undergraduate sample, Zayco (2009) found that greater loss of face concerns predicted less favorable counseling attitudes, including less emotional openness and greater stigma concerns. In a similar investigation, Chou (2000) examined loss of face as a predictor of Asian American undergraduates' preferences for a culturally and ethnically matched therapist. In this study, Asian American students who experienced greater loss of face fears also held a stronger preference for an ethnically similar counselor, as well as for a counselor with similar cultural values. Finally, in an epidemiological study of Filipino Americans' mental health service use, Gong, Gage, and Tacata (2003) observed that participants who endorsed greater loss of face fears were significantly less likely to have utilized counseling in the past. This effect persisted even after controlling for demographic variables, such as age, gender, generation status, and overall psychological distress. Thus, 
preliminary findings indicate that loss of face concerns may serve as a significant barrier to mental health service use among Asian and Asian American populations.

\section{Critical Issues in the Existing Literature}

The literature reviewed above points to the possible interplay of several cognitive and cultural factors in explaining Asian international students' reduced use of professional counseling. This body of literature, however, has been frequently limited by several pervasive methodological and conceptual problems. One particular problem is combining foreign-born students from Asia with samples of international students from other geographic regions (Yoon \& Portman, 2009). Specifically, a great number of the studies cited in this brief literature review focused on international students in general, rather than on foreign-born students from Asia specifically (e.g., Anderson \& Myer, 1985; Nilsson et al., 2004; Mitchell et al., 2007; Yakushko et al., 2008). Relatively few studies have examined the counseling service utilization patterns and help-seeking intentions of Asian international students as a group in its own right (e.g., Dadfar \& Friedlander, 1982; Mau \& Jepsen, 1990; Tedeschi \& Willis, 1993).

The trend of combining Asian international students with foreign-born students from other regions is problematic because doing so may obscure important differences based on national heritage and cultural background. For example, Fritz, Chin, and DeMartinis (2008) found that when international students were compared to U.S.-born undergraduates in terms of overall adjustment level, no significant differences could be found. However, when international students were divided into subsamples based on national origin (i.e.., European and Asian), important within-group differences emerged. Specifically, the Asian international students experienced significantly greater anxiety 
and adjustment problems than did the European international and U.S.-born American students. As a result, the above authors argued that "important differences between cultural groups can be missed when international students are classified as one homogenous group" (p. 251). These authors also suggested that future research should examine distinct cultural subgroups (e.g., foreign-born students from Asia), instead of treating international students as a homogenous population.

A second potentially problematic trend is to combine foreign-born Asian international and U.S.-born Asian American undergraduates within the same sample (Yoon \& Portman, 2009). For example, Liao et al. (2005) combined U.S.-born Asian American and foreign-born Asian international undergraduates, while failing to explore potential differences between the two groups of students. A related limitation has been the trend to generalize findings obtained from samples of U.S.-born Asian American participants to the somewhat distinct population of foreign-born Asian international students. For example, much of the research on cultural predictors of counseling service use has relied on U.S.-born Asian American samples, rather than on foreign-born Asian international participants (e.g., Atkinson \& Gim, 1989; Chou, 2000; Gloria et al., 2008; Gong et al., 2003; Kim, 2007; Miville \& Constantine, 2007; Shea \& Yeh, 2007; Tata \& Leung, 1994; Zayco, 2009 ). In fact, I am aware of only one study that examined acculturation as a predictor of help-seeking among Asian international students specifically (e.g., Zhang \& Dixon, 2004), and of no studies that explored Asian values and loss of face concerns among this population.

The tendency to overgeneralize findings from Asian American to Asian international students is problematic because the two groups differ in terms of reasons for 
immigration, immigrant status, acculturation, and adjustment experiences in the U.S. For example, Sodowsky and Plake (1992) found that Asian international students experienced greater language difficulties, more cultural adjustment problems, and higher levels of discrimination, as compared to U.S.-born Asian American students. The Asian international students also were significantly less acculturated and experienced greater cultural adaptation difficulties than their U.S.-born Asian American counterparts. Furthermore, Coppi (2007) noted that Asian international students are defined as "legal aliens" by the U.S. Customs and Immigration Services, whereas U.S.-born Asian Americans typically have permanent resident or citizen status. Thus, because of their immigrant status, Asian international students may experience greater adaption challenges than do their U.S.-born Asian American counterparts. Given these considerations, it has been argued that researchers should not overgeneralize findings from Asian American to Asian international student populations (Yoon \& Portman, 2009). In order to avoid such overgeneralizations, researchers need to study Asian international students as a group in its own right.

A third problem in the existing literature is the limited use of theoretical frameworks to guide empirical inquiry (Mori, 2000; Pedersen, 1991). As Pedersen (1991) observed, the international student research literature has been characterized by "isolated, uncoordinated, and fragmentary studies on specialized variables with no clear application of results to comprehensive theory building" (p. 50). In fact, to my knowledge, to date only one study has used an a priori specified theoretical model to examine Asian American and Asian international students' help-seeking (Liao et al., 2005). The remaining research literature is largely exploratory and atheoretical in nature, such that 
variables are selected without a solid rationale, and studies explore different constructs in isolation without considering complex and theoretically grounded empirical questions (e.g., Dadfar \& Friedlander, 1982; Brinson \& Kottler, 1995; Kindaichi et al., 2007; Mau \& Jepsen, 1990; Tedeschi \& Willis, 1993; Yoon \& Jepsen, 2008; Zhang \& Dixon, 2003). The lack of theoretical frameworks in the literature reviewed above is problematic, given the importance of theory for conceptualizing, implementing, and applying empirical research. It has repeatedly been argued that theory building is necessary in order for researchers to address meaningful research questions and select relevant psychological constructs (Karr \& Larson, 2005). It also has been suggested that theory is needed in order to create research literature that is conceptually sound and clinically applicable. For example, Murdock (2006) suggested that theory is needed in order to generate empirical findings that have practical significance and applicability to real life, thus facilitating greater science-practice integration. Given the importance of a guiding theoretical framework, future research on Asian international students' helpseeking should be guided by a conceptually sound theoretical model (Pedersen, 1991). Such a theoretical model should be able to integrate both cognitive and cultural predictors of Asian international students' counseling service utilization (Heggins \& Jackson, 2003; Ibrahim \& Ingram, 2007; Johnson \& Sandhu, 2007; Sheu \& Fukuyama, 2007).

\section{The Theory of Reasoned Action as a Model}

One conceptual framework that has the potential to integrate both cognitive and cultural variables in the prediction of help-seeking behavior is the Theory of Reasoned Action (TRA, Ajzen \& Fishbein, 1980; Fishbein \& Ajzen, 2009). The TRA was originally developed in the early 1980s with the goal of explaining and predicting a wide 
range of volitional behaviors, such as voting, energy conservation, and help-seeking (Fishbein \& Ajzen, 2009). The theory assumes that people generally act in thoughtful, systematic, and rational ways by considering their intentions, attitudes, and social norms before engaging in action. Given the assumption of a "reasoned" action approach, Ajzen and Fishbein (1980) and Fishbein and Ajzen (2009) suggested that it is possible to predict and understand a person's behavior by examining a limited number of cognitive predictors. The three cognitive variables offered by these authors as the best and most immediate predictors of future actions were intentions, attitudes, and subjective norms (Ajzen \& Fishbein, 1980; Fishbein \& Ajzen, 2009) (see Figure 1.1).

Under the TRA, intentions are defined as "the motivational factors that influence a behavior" or as "indicators of how hard people are willing to try in order to perform a behavior." According to the TRA, intentions are hypothesized to be the best available predictor of actual behavior, such that "barring unforeseen events, a person will usually act in accordance with his or her intentions" (Ajzen \& Fishbein, 1980, p. 5). Intentions are, in turn, predicted by the individual's attitudes and subjective norms. Ajzen and Fishbein (1980) defined attitudes as "the individual's positive or negative evaluation of performing the behavior" or as "the person's judgment that performing a behavior is good or bad" (p. 6). In contrast, subjective norms refer to "the person's perception of the social pressures put on him or her to perform or not perform the behavior in question" ( $p$. 6). It is important to note that, according to the TRA, an individual's attitudes and subjective norms predict behavior only indirectly, through the mediating effect of intentions (Ajzen \& Fishbein, 1980; Fishbein \& Ajzen, 2009; see Figure 1.1). 
Figure 1.1 Theory of Reasoned Action Model

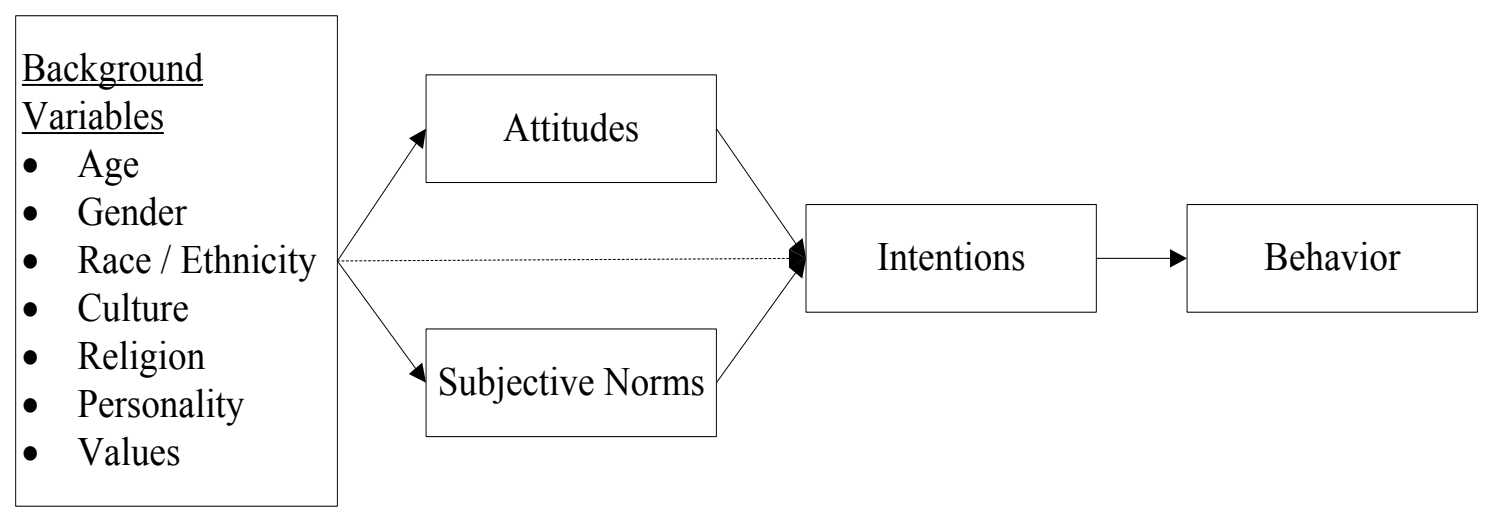

Although the TRA focuses primarily on cognitive variables as predictors of human behavior, the theory also recognizes the impact of demographic and cultural factors (e.g., age, gender, race/ethnicity, culture, etc.; Ajzen \& Fishbein, 1980; Fishbein \& Ajzen, 2009). Specifically, Fishbein and Ajzen suggested that cultural and demographic variables may influence human behavior indirectly through their impact on attitudes, subjective norms, and behavioral intentions. Fishbein and Ajzen chose not to provide a comprehensive list of different background variables that could be relevant in a TRA model, stating that these would vary based on the specific behavioral domain and the individual cultural group under investigation. However, it is clear from the model presented in Figure 1.1 that cultural experiences, expectations, and values can be easily integrated in a TRA-based help-seeking model (Fishbein \& Ajzen, 2009).

Attention to the TRA as a framework for understanding help-seeking behavior has grown considerably in recent years. For example, Christopher, Skillman, Kirkhart, and D'Souza (2006) used the TRA as a preventive model for increasing psychological helpseeking among Thai and American undergraduates. These authors found that cultural background (i.e., Thai or American) predicted the type of persuasive message that was 
most effective in increasing participants' intentions to seek counseling. Specifically, American students were most strongly influenced by a persuasive message that targeted their attitudes, whereas Thai participants were most strongly influenced by a message that altered their subjective norms. Similarly, Mo and Mak (2009) observed that the TRA offers a good conceptual framework for predicting mental health help-seeking in a community sample of Hong-Kong Chinese. In this study, participants' attitudes and subjective norms accounted for $57 \%$ of their intentions to seek counseling in the event of a psychological problem. Finally, Yong (2006) found that the TRA offers a good theoretical model for understanding Chinese American parents' intentions to seek psychological services for their children. Consistent with the TRA premises, both cognitive variables (i.e., attitudes and subjective norms) and cultural factors (i.e., acculturation) were predictive of help-seeking intentions among this population.

Although the TRA has been gradually receiving recognition as a help-seeking framework, I could identify no research testing the TRA premises with an Asian international student population. To my knowledge, no theoretical or empirical writing has previously considered the TRA as a model for understanding Asian international students' intentions to seek counseling. In addition, I am aware of no studies that have integrated both cognitive predictors (i.e., help-seeking intentions, counseling attitudes, and stigma concerns) and cultural variables (i.e., acculturation, Asian values, and loss of face concerns) in predicting Asian international students help-seeking. Finally, to my knowledge, no study to date has examined stigma concerns as a subset of subjective norms, using a TRA-based help-seeking framework. Given the need to integrate both cognitive and cultural predictors of help-seeking in a theoretically sound conceptual 
model, future research should examine the TRA as a help-seeking framework with an Asian international student population.

\section{The Purpose of the Present Study}

Given the directions for future research presented above, the purpose of the present study was to present a theoretical model combining both cognitive and cultural variables affecting Asian international students' intentions to seek counseling. The TRA (Ajzen \& Fishbein, 1980; Fishbein \& Ajzen, 2009) was proposed as a unifying theoretical model for understanding Asian international students' help-seeking. The theory is suitable for examining the cognitive antecedents of help-seeking behavior (e.g., help-seeking intentions, attitudes, and stigma), while also allowing for the inclusion of important cultural factors (i.e., acculturation, Asian cultural values, and loss of face). The theoretical model that was explored in this study is presented in Figure 1.2.

Figure 1.2 Theoretical Model Guiding the Present Study

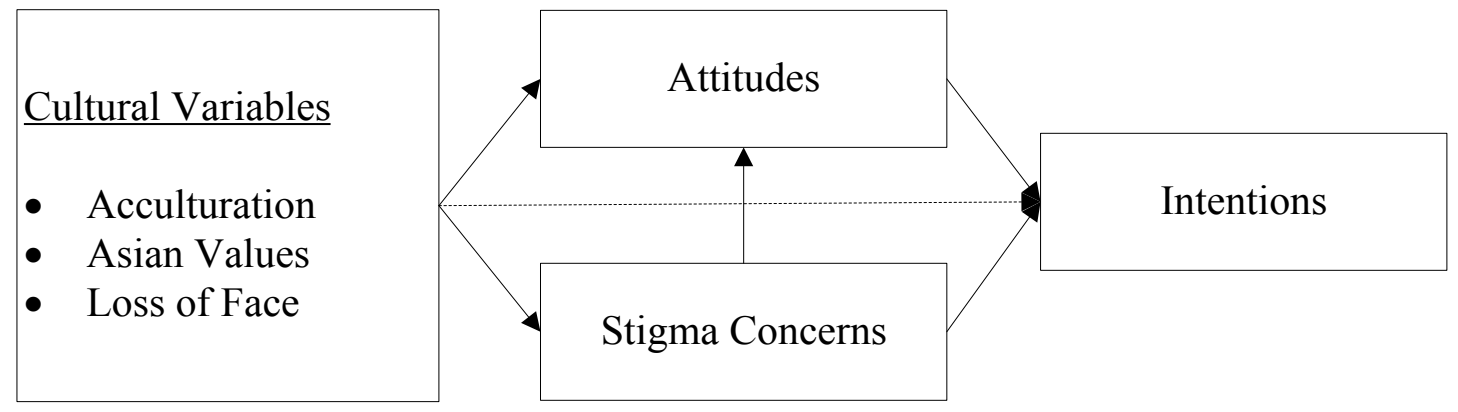

Consistent with the TRA premises, it was expected that Asian international students' counseling attitudes and stigma concerns will emerge as significant predictors of their intentions to seek counseling for a personal-emotional problem. Furthermore, it was hypothesized that cultural variables (e.g., acculturation, Asian values, and loss of face concerns) will also emerge as significant predictors of Asian international students' intentions to seek counseling. The effect of these cultural variables, however, was 
hypothesized to occur through the mediating influence of counseling attitudes and stigma concerns, as indicated by a TRA-based help-seeking model (Ajzen \& Fishbein, 1980; Fishbein \& Ajzen, 2009). It was hypothesized that the resulting conceptual model will offer a good fit for the data in terms of predicting Asian international students' intentions to use counseling.

The results of this study have the potential to inform future research and practice. In terms of research, this study could provide a theoretical framework that can be further tested and refined in several consecutive empirical investigations. Furthermore, the results of this study could encourage future research on multiple cognitive and cultural predictors of counseling use among Asian international students in particular and culturally diverse populations in general. In terms of their applicability to clinical practice, the results of this study could provide a theory-driven model that could guide future preventative work with Asian international students. Specifically, a TRA-based model could be used to design preventive psychoeducational programs with the aim of increasing help-seeking behavior among this population. Thus, the results of this study may contribute to both research and practice, while stimulating greater attention to several cognitive and cultural predictors of psychological help-seeking.

\section{Definitions of Terms}

Given the importance of conceptual clarity in the use of psychological terms, it is important to define the constructs of interest explored in this study (Tabachnick \& Fidell, 2007). To that effect, a brief list of construct definitions is presented in Table 1.1 below. These definitions are drawn from the existing theoretical literature and pertain to the way each term will be defined and used in the present study. 
Table 1.1 Important Construct Definitions

\begin{tabular}{ll}
\hline Construct & Definition \\
\hline American Student & $\begin{array}{l}\text { A college student who holds a U.S. permanent resident or } \\
\text { citizen status and endorses one of the following ethnicities: } \\
\text { European American, Latino/a American, African } \\
\text { American, Asian American, Native American, or other } \\
\text { (U.S. Census Bureau, 2007). }\end{array}$ \\
& $\begin{array}{l}\text { A college student who holds a foreign student visa (i.e., F- } \\
\text { International student } \\
\text { students in the U.S. come from diverse geographic regions, } \\
\text { including Africa, Asia, Europe, Latin America, the Middle } \\
\text { East, North America, and Oceania (Institute of } \\
\text { International Education, IIE, 2010). }\end{array}$
\end{tabular}

Asian American student A college student who holds a U.S. permanent resident or citizen status and who endorses an Asian American background (Coppi, 2007; U.S. Census Bureau, 2007).

Asian international student

An international student who comes from one of the following geographic regions: 1) East and Southeast Asia; 2) South and Central Asia. International students from the Middle East are excluded from this definition, given their unique geographic and cultural background (IIE, 2010; U.S. Census Bureau, 2007).

Intentions to seek counseling

Individuals' future-oriented intentions to seek professional psychological counseling in the event of experiencing a personal-emotional problem (Ajzen \& Fishbein, 1980; Cash, Begley, McCown, \& Weise, 1975).

Attitudes toward seeking counseling

Individuals' cognitive-affective evaluation of the act of seeking counseling for a personal-emotional problem. Attitudes have an evaluative component, such that seeking counseling is seen as either good or bad, desirable or undesirable, appropriate or inappropriate (Fischer \& Farina, 1995; Fischer \& Turner, 1970). 
Counseling stigma concerns

Acculturation

Asian cultural values

Loss of face concerns
Individuals' concerns that they would be stigmatized or judged negatively by significant others in the event of seeking professional counseling (Vogel, Wade, \& Ascheman, 2009). In this study, stigma concerns are conceptualized as a subset of individuals' subjective social norms, such that seeking counseling can be seen as potentially stigmatizing (Ajzen \& Fishbein, 1980).

The process of behavioral change that occurs when an individual is exposed to a new culture. Acculturation refers to the degree to which an individual comes to adopt the host culture's language, customs, norms, and traditions (Kim \& Abreu, 2001; Chung, Kim, \& Abreu, 2004).

The value orientations endorsed by many traditional Asian cultures (e.g., Japanese, Chinese, Asian Indian, Vietnamese, etc.). These value orientations are typically characterized by a strong emphasis on collectivism, conformity to norms, emotional self-control, family recognition through achievement, filial piety, and humility (Kim, Atkinson, \& Yang, 1999; Kim, Lin, \& Ng, 2005).

In many traditional Asian cultures (e.g., Chinese, Japanese, Asian Indian), "face" refers to the individual's moral and social character in the Asian community. Loss of face can occur if the individual breaches important social norms or compromises his or her moral integrity. In this context, the term "loss of face concerns" refers to individuals' fears that they will lose their social status or moral image in the Asian community (Ho, 1991; Zane \& Yeh, 2002). 


\section{CHAPTER II}

\section{REVIEW OF THE LITERATURE}

The purpose of this chapter is to provide a literature review for the proposed study on Asian international students' intentions to seek counseling. I will first describe Asian international students as a population, followed by a review of the literature on their use of counseling services. I will then discuss empirical findings on three cognitive predictors of counseling service use: intentions, attitudes, and social stigma concerns. Subsequently, I will review research on three cultural variables that may be particularly relevant to this population: acculturation, Asian values, and loss of face concerns. Finally, I will discuss the Theory of Reasoned Action (TRA, Ajzen \& Fishbein, 1980; Fishbein \& Ajzen, 2009) as an integrative framework for these cognitive and cultural constructs. I will conclude the chapter with a list of hypotheses that provide the background for the present study.

\section{Asian International Students in the U.S.}

The number of international students in the U.S. has increased twenty six times over the last six decades, from 47,000 international students during the 1948-1949 academic year to 671,000 during 2008-2009. Over the same time period, the percentage of international students increased from $1.1 \%$ to $3.7 \%$ of the total U.S. college student enrollment (Institute of International Education, IIE, 2010). In 2008-2009, more than half of all enrolled international students came from the Asian continent, with India $(103,260$ students), China $(98,235)$, South Korea $(75,065)$, Japan $(29,264)$, Taiwan $(28,065)$, and 
Vietnam $(12,823)$ ranked among the top ten countries sending the largest numbers of international students to the U.S. Other Asian countries with a large representation among international students included Nepal (11,581 students), Thailand (8,736), Hong Kong $(8,329)$, Indonesia (7,509), Malaysia $(5,942)$, and Pakistan (5,298; IIE, 2010). A quick glance at the statistics reported above indicates that Asian international students come from a range of different countries and as such represent a heterogeneous and culturally diverse group.

Given the extensive cultural diversity represented among Asian international students, it is difficult to provide a prototypical example that can uniformly represent this population (Sheu \& Fukuyama, 2007). Several broad generalizations can be made, however, based on current educational statistics and recent empirical research. During the 2008-2009 academic year, for example, a majority of Asian international students (46.8\%) were pursuing advanced graduate training, including degrees at the master's (23.8\%), doctoral (17.9\%), and professional level (5.1\%). In addition, during the same academic year, 32.4\% of Asian international students pursued bachelor degrees, $12.2 \%$ sought associate degrees, and $8.6 \%$ were enrolled in intensive English language programs. The most popular academic fields of study included business and management (20.6\%), engineering (17.7\%), liberal arts and sciences (10.9\%), physical and life sciences $(9.2 \%)$, social sciences $(8.5 \%)$, and mathematics and computer science $(8.4 \%)$. Less common fields of study included fine and applied arts (5.2\%), the humanities (2.9\%), education (2.7\%), and agriculture (1.3\%; IIE, 2010). These statistics indicate that, generally speaking, Asian international students are a highly selected and well-educated group of students. In addition, a majority of Asian international students pursue advanced 
degrees in business and the STEM professions (science, technology, engineering, and mathematics).

In terms of primary sources of financial funding, the majority of Asian international students were supported by personal and family funds (64.9\%), U.S. college or university stipends $(22.7 \%)$, current employer (5.0\%) and home government funds (3.7\%). Less common sources of financial support included a U.S. private sponsor (1.1\%), a foreign private sponsor $(0.9 \%)$, the U.S. government $(0.6 \%)$, and non-profit organizations $(0.2 \%)$. This suggests that a large proportion of Asian international students may come from a relatively privileged socioeconomic background, given that a substantial majority was able to fund their study in the U.S. through personal and family funds. Furthermore, in terms of distribution across the U.S., the top ten states with the highest numbers of international students included California $(93,124)$, New York (74,934), Texas $(58,18)$, Massachusetts $(33,838)$, Florida $(30,386)$, Illinois $(29,887)$, Pennsylvania (27,529), Michigan (23,617), Ohio (20,725), and Indiana (17,098). Finally, the top five U.S. institutions with the greatest population of international students included the University of Southern California (7,482 students), New York University $(6,761)$, Columbia University $(6,685)$, University of Illinois at Urbana-Champaign (6,570), and Purdue University (6,136, IIE, 2010).

Apart from the educational statistics presented above, research indicates that Asian international students encounter a number of stressors beyond the normal developmental challenges of the college transition. These unique difficulties include a language barrier, loss of social support, discrimination, and acculturative stress. Constantine and colleagues (2005), for example, observed that language difficulties were 
one of the most challenging issues faced by Asian international students. In this study, language problems negatively impacted Asian international students' academic performance in American classrooms, which in turn was predictive of higher levels of psychological distress. Similarly, Surdham and Collins (1984) found that Asian international students who believed that their English language skills were inadequate at the time of their arrival in the U.S. tended to experience less social support from their American peers and to report more adjustment difficulties. Furthermore, in a series of three qualitative studies, Swagler and Ellis (2003) found that communication difficulties and lack of confidence in one's ability to speak English were some of the most salient adjustment challenges for Taiwanese international students. Specifically, participants with lower English fluency and higher communication concerns tended to experience more acculturative stressors and lower adjustment to life in the U.S.

Loss of social support is another salient issue for Asian international students. Constantine and colleagues (2005) found that Asian international students often report social isolation, due to the loss of social support provided by friends and family back home. In fact, a substantial majority of Asian international students reported persistent feelings of homesickness, loneliness, and social isolation upon their arrival in the U.S., paired with missing family, friends, and significant others in the home country. Furthermore, Olivas and $\mathrm{Li}$ (2006) found that Asian international students experienced significant concerns with not being able to fit in and build friendships with their American peers. Asian international students were especially concerned about cultural misunderstandings, language difficulties, and possible discrimination as barriers to forming American friendships. Further research indicates that lack of social support is 
frequently associated with lower psychological adjustment and greater depressive symptoms among Asian international students (Ying \& Liese, 1994). Finally, Misra, Crist, and Burant (2003) found that foreign students from Asia who experienced lower levels of social support were less able to cope with the academic and acculturation stressors of study abroad, resulting in greater psychological distress.

Possible racial and cultural discrimination also has been cited as a common adjustment challenge for Asian international students. Heggins and Jackson (2003) found that many Asian students report not feeling welcomed and being treated like uninvited guests in a strange land. Similarly, in their qualitative research, Swagler and Ellis (2003) discovered that many Taiwanese international students experienced encounters with racial discrimination, paired with being seen as outsiders or not fitting in. Research findings also indicate that encounters with discrimination are linked with greater adjustment difficulties and lower psychological health among international students in general and foreign students from Asia specifically. Wadsworth, Hecht, and Jung (2008), for example, found that Asian international students who experienced greater racial discrimination also reported lower adjustment to the American educational system. Jung, Hecht, and Wadsworth (2007) similarly found that Asian international students who experienced more racial discrimination tended to report higher depression symptoms and lower psychological adjustment. Finally, Sodowsky and Plake (1992) found that Asian international students as a group experienced significantly more cultural and racial discrimination than did their U.S.-born Asian American counterparts.

Finally, Asian international students also report high levels of acculturative stress, which is defined as the psychological tension experienced during the process of 
acculturation (Johnson \& Sandhu, 2007). Yeh and Inose (2003) found, for example, that acculturative stress is common among Asian international students who also experience greater level of acculturation-related difficulties, as compared to international students from Europe. Poyrazli, Kavanaugh, and Baker (2004) similarly found that, compared with foreign students from Europe, Asian international students reported more acculturative stress, possibly due to greater differences in cultural values and more frequent exposure to racial discrimination. Research also indicates that acculturative stress is associated with greater psychological distress among international students in general and foreign students from Asia specifically. Constantine, Okazaki, and Utsey (2004) found that international students who experienced greater acculturation stress also reported more depressive symptoms. Similarly, Wilton and Constantine (2003) observed that international students who endorsed greater acculturative stress also experienced greater psychological maladjustment, including more symptoms of depression and anxiety. Finally, in a study by Misra and colleagues (2003), Asian international students who experienced greater acculturation stress also reported significantly greater levels of somatic, stress-related symptoms.

Given the unique adjustment challenges and acculturation stressors described above, several authors have suggested that counseling may offer a supportive network that can facilitate Asian international students' adjustment in the U.S. Johnson and Sandhu (2007) suggested, for example, that counseling can provide opportunities to practice English in a non-evaluating context as well as help international students experiment with new interpersonal skills and ways to build social support. Counseling also can provide a safe place to discuss encounters with racial discrimination, and 
counselors can help international students develop new strategies for coping with ethnic prejudice and stereotypes (Lin \& Pedersen, 2007). Finally, counseling can help students cope with the experience of acculturative stress, thus reducing depression, anxiety, and psychological distress (Ibrahim \& Ingram, 2007; Morri, 2001; Sheu \& Fukuyama, 2007). Despite these potential benefits of counseling, however, research indicates that international students in general, and Asian international students specifically are less likely to seek counseling than American students. The next section of this chapter discusses current empirical findings on Asian international students' counseling use.

\section{Asian International Students' Counseling Use}

In recent years, studies have examined the counseling use of international students in general (e.g., Anderson \& Myer, 1985; Nilsson, Berkel, Flores, \& Lucas, 2004; Yi, Lin, \& Kishimoto, 2003) and of Asian foreign students specifically (e.g., Huyn, Quinn, Madon, \& Lustig, 2007; Mitchell, Greenwood, \& Gugliemi, 2007; Yakushko, Davidson, \& Sanford-Martens, 2008). These studies have tended to compare international students' counseling utilization, presenting concerns, referral sources, number of sessions attended, and no-show and premature termination rates against those of American students. In addition, three studies (e.g., Huyn et al., 2007; Mitchell et al., 2007; Yakushko et al., 2008) also examined group differences within the international student population based on continent of origin, thus providing separate data for Asian foreign students. Because to date only three investigations have specifically examined Asian international students' counseling use, I will first review studies using international students' samples in general. I will then focus on the three studies that examined Asian international students specifically, highlighting those as most relevant for the purpose of the present study. 
One study that examined international students' utilization of college counseling in general was conducted by Anderson and Myer (1985). These authors examined the patterns of presenting concerns, counselor contacts, and no-shows for international and American students seeking services at a college counseling center. Data were gathered from 40 international and 40 American students who received services at the center during one academic year. Between-group comparisons indicated that international and American students visited the counseling center with similar presenting concerns (e.g., anxiety, depression, relationship problems, and academic difficulties), $\chi^{2}(1)=1.25, p>$ .05. However, unlike American students, international students typically failed to return for counseling after the intake appointment, $\chi^{2}(1)=4.46, p<.05$. Specifically, $33 \%$ of the international clients failed to keep a second appointment, as compared to $12 \%$ of American clients. In fact, the international students had a higher no-show ratio (1 out of 7 sessions) than did American students (1 out of 11 sessions). A significant limitation of this study is that the authors failed to report the ethnic background and the country of origin for the 40 international students in this sample. As a result, the actual number of Asian international students who were represented in the sample is unknown. In a similar study, Nilsson and colleagues (2004) examined the presenting concerns and utilization rates of international clients seeking services at a university counseling center over a one-year period. Data were obtained from the client files of 41 foreign born students who utilized services during the selected time period. The majority of these students identified as Asian (65.9\%), followed by European (19.5\%), Latino/a (7.3\%), African (2.4\%), and other (4.9\%). Available data indicated that, although international students constituted $8 \%$ of the total student body, they made up only $2.6 \%$ 
of the clients seen at the counseling center. In comparison, U.S.-born African American students constituted $13 \%$ of the student body and $15 \%$ of the clients, U.S.-born Asian American students represented $12 \%$ of the student body and $13 \%$ of the clients, and U.S.born Hispanic American students comprised 4\% of the student body and 5\% of the clients seen at the counseling center over the same time period. Thus, international students had lower counseling use rates, as compared to U.S.-born minority students. A limitation of this study was that the authors aggregated the utilization rates of foreign students from different geographic regions, thus failing to provide statistics for Asian students. In addition, the authors failed to conduct more detailed statistical analyses, leaving it unclear if the reported differences in utilization rates were statistically significant.

In another study, Yi and colleagues (2003) examined the utilization patterns of 516 international students receiving services at a university counseling center during a 6year period (1992-1998). Asian students comprised over one-half (59\%) of the sample, followed by students from Central and South America (24.1\%) and Europe (10.2\%). Of the Asian countries represented, the leading countries of origin were China $(n=65)$, India $(n=54)$, Japan $(n=51)$, Taiwan $(n=27)$, and Korea $(n=21)$. Referral sources for the students in this sample were as follows: self-referred (57.5\%), referred by an academic advisor or staff (22.1\%), referred by friends or family $(13.0 \%)$, and referred by faculty (5.4\%). The five most frequent presenting problems were academic concerns (39.4\%), depression (31.5\%), anxiety (31.3\%), confusion about one's value system (28.1\%), and self-esteem (28.0\%). After running within-group analyses for the international student sample, these authors found no significant relationships between students' continent of origin and type of referral source or presenting concern. However, the authors failed to 
collect data about participants' total number of attended sessions, no-show and premature termination rates, and reasons for terminating therapy. As a result, it was impossible to make within-group comparisons based on these important dimensions of interest. Another significant limitation of this study is that the authors failed to compare international students' utilization rates versus those of American students, making it impossible to examine differences between international and American students.

In a study that focused on Asian international students specifically, Huyn and coauthors (2007) compared the counseling center utilization rates of international and American graduate students at a large, Western university. All registered graduate students with a valid e-mail address were invited to participate in an online survey, with 551 international students responding to the survey. Of the international student respondents, $50.8 \%$ self-identified as Asian, $36.1 \%$ as European, $7.5 \%$ as Latin American, $1.9 \%$ as Middle Eastern, $0.6 \%$ as African, and 3.2\% as other. These students were compared to a corresponding sample of American graduate students $(N=2,493)$ who completed the same online survey. Results indicated that, although the prevalence of emotional problems was similar for both groups ( $44 \%$ vs. $46 \%$, respectively), international students were significantly less likely to have considered using mental health services (33\% vs. $56 \%$, respectively) or to have sought counseling in the past $(17 \%$ vs. $36 \%$, respectively), $\chi^{2}(1, N=3,023)=67.98, p<.01$. In addition, logistic regression analyses indicated that Asian international students were significantly less likely to seek counseling as compared to foreign-born students from Africa, Latin America, and Europe $(O R=0.19, p<.01)$. A particular strength of this study is that the authors successfully 
examined within-group differences in the international student population, thus focusing on foreign-born Asian students specifically.

Another study that specifically examined Asian international students' counseling use was conducted by Mitchell and colleagues (2007). These authors studied the counseling center utilization patterns of 218 international and 222 U.S.-born college students during a 2-year period. The foreign students in this sample represented 52 countries, with 67\% coming from Asia, 12\% from North America, 11\% from Europe, and the remaining 10\% from Africa, South America, and Australia. Counseling center records were examined to collect data about participants' referral source, number of sessions attended, and no-show and premature termination rates. Data indicated that the international students were significantly more likely to be referred by faculty or staff (30\% vs. $15 \%)$ and significantly less likely to self-refer for counseling (48\% vs. $60 \%)$ than were American students, $\chi^{2}(3, N=431)=20.56, p=.001$. International students also were less likely than American students to have had prior counseling experience $(32 \%$ vs. $55 \%), \chi^{2}(3, N=425)=23.07, p=.001$. Furthermore, Asian international students were found to attended significantly fewer sessions $(M=3.73, S D=5.12)$ than did foreign students from Europe $(M=6.92, S D=6.45), p=.05$. A particular strength of this study was to examine both between-group and within-group differences, thus highlighting important variations based on continent of origin (i.e., Asia vs. Europe).

Another study that specifically focused on Asian international students was conducted by Yakushko and coauthors (2008). These authors examined foreign students' use of mental health services at a single university counseling center during a 5-year period (1996-2001). One hundred and thirty-two foreign students sought counseling 
during the selected period, comprising only $1.8 \%$ of the foreign student body enrolled at the university. Of the 132 international students in this sample, the majority came from Asia and the Pacific Islands (46\%), followed by Europe, Australia, and Canada (19\%), Central and South America (14\%), the Middle East (11\%), and Africa (10\%). Descriptive analyses indicated that international students tended to use counseling services for very brief periods, with $36 \%$ attending only one counseling session. The most common termination pattern (29\%) was a client no-show. Furthermore, Asian international students attended significantly fewer sessions than did students from Central and South America, $F(4,122)=3.21, p<.02$. In addition, Asian students were less likely to selfrefer for counseling than were international students from other regions, $\chi^{2}(4, N=122)=$ $13.54, p=.009$. A strength of this study was the fact that these authors examined withingroup differences, with a focus on foreign-born students from Asia. However, an important limitation was the failure to include a sub-sample of U.S.-born students in order to allow meaningful between-group comparisons.

Taken collectively, these studies suffer from a number of methodological limitations. Most used extremely small sample sizes (Anderson \& Myer, 1985; Nilsson et al., 2004; Yakushko, et al., 2008), which may have resulted in insufficient statistical power (Tabachnick \& Fidell, 2007). Several investigations failed to make between-group comparisons of international and American students (e.g., Yi et al., 2003; Yakushko et al., 2008). On the other hand, several studies that did examine between-group differences failed to also explore possible within-group differences based on international students' region of origin (e.g., Anderson \& Myer, 1985; Nilsson et al., 2004). As a final limitation, all of these studies used archival data sets, which limited the type of available 
data. Specifically, most studies examined the relationships among demographic variables, presenting concerns, referral source, number of attended sessions, and premature termination rates for international students in general, and Asian foreign students in particular. Other predictors of counseling utilization, such as cognitive variables (e.g., intentions, attitudes, and stigma concerns) and cultural factors (e.g., acculturation, Asian values, and loss of face), need to receive greater attention. The next sections of this literature review address each of these cognitive and cultural predictors individually.

\section{Intentions to Seek Psychological Counseling}

Intentions to seek professional counseling are a key cognitive factor hypothesized to predict counseling use among Asian international students (Lippincott \& Mierzwa, 1995; Mau \& Jepsen, 1990). Intentions have been previously defined as “a person's motivation in the sense of her or his conscious plan or decision to exert effort toward performing a behavior" (Conner \& Norman, 1996). In the literature, intentions have been conceptualized as an immediate precursor of behavior, such that intentions to seek counseling may be one of the best indicators of future counseling use. Several authors have written about the association between intentions and future behavior in general (Fishbeing \& Ajzen, 2009; Kim \& Hunter, 1993), and intentions to seek counseling and future utilization of counseling services in particular (Wilson, Deane, Ciarrochi, \& Rickwood, 2005). Kim and Hunter (1993), for example, found a moderate association ( $r$ $=.46$ ) between intentions and behavior in a meta-analytic review of 47 studies. Dean and colleges (2005) similarly found that college students' intentions to seek counseling predicted help-seeking behavior three weeks later. Thus, intentions to seek counseling may be an important predictor of counseling use among Asian international students. 
Given the potential significance of intentions as predictors of help-seeking behavior, it is important to study this construct with Asian international students. Mau and Jepsen (1990) conducted one of just a few such studies using a sample of 102 Chinese international and 148 American students. All participants rank-ordered the ideal helper (i.e., friend, parent, academic advisor, counselor, clinical psychologist, psychiatrist, physician, priest, spouse, or no one) from whom they would seek help for a psychological problem. Analyses indicated that Chinese international students were significantly less likely to seek help from a mental health professional, $t(244)=-5.59, p$ $<.001$. Furthermore, Chinese international students (24.8\%) favored "no one" as their top ranked helper, as compared to American students (31.8\%) who favored a psychiatrist. These results indicate that Chinese students may view seeking counseling more negatively than do American students, and thus may be less likely to utilize mental health services. In addition, in the event of a psychological problem, Chinese international students may fail to access even informal social support, underscoring the need to promote help-seeking among this population. A limitation of this study, however, was the authors' failure to report students' rankings of available helpers beyond the most frequently selected option. Thus, it is unclear what proportion of Chinese students indicated that they would seek help from a mental health professional.

In another study, Lippincott and Mierzwa (1995) examined Asian international and American students' intentions to seek counseling for somatic concerns. The authors recruited 20 Asian international and 39 American undergraduate students. The countries of origin for the Asian students included China, Hong Kong, Malaysia, Indonesia, Singapore, Korea, the Philippines, Taiwan, Thailand, India, Pakistan, and Sri Lanka. All 
participants completed a modified somatic symptoms checklist (the Brief Symptom Inventory) and a measure of intentions to seek counseling for somatic problems that was created for the purpose of the study. Analyses indicated that the Asian international students $(N=20 ; M=66.85 ; S D=10.22)$ reported significantly greater intentions to seek counseling for somatic concerns $(t=3.35, p<.005)$ than did American students $(N=39$; $M=56.85 ; S D=12.11)$. The authors concluded that these results provide preliminary evidence for Asian international students' increased likelihood to seek counseling for somatic complaints. A limitation of this study was the small sample size and the use of modified and unvalidated questionnaires. In addition, the authors failed to include a measure of participants' intentions to seek counseling for psychological problems; thus, the degree to which Asian international students are willing to seek mental health services for emotional problems remains unknown.

In another study, Brinson and Kottler (1995) used a qualitative design by interviewing 100 international students about their perceptions of the counseling services available at U.S. colleges and universities. Data obtained from participants' semistructured interviews indicated that international students may view counseling negatively. For example, one 25-year-old male international student from Hong Kong expressed strong negative perceptions and low intentions to seek counseling, as revealed in the following quote: "In Hong Kong, counseling a bad thing. Chinese students never, never go to counseling by ourselves. Only if teachers send you there. Counseling bad, for weak people. Problem child. If friends know you go to counseling, they stay away from you" (p. 60). Similarly, a 25-year-old female international student from Korea stated: "I know there is a student counseling center, but I never think about go there. Too far from 
me. Center just for normal American students, not foreign students" (p. 63). These exemplar quotes reveal that some Asian international students may view counseling negatively, and, thus, may have low intentions to access college counseling services. The findings of this study are limited, however, by the authors' failure to provide sample demographic data, leaving the number of Asian participants unknown. In addition, the authors failed to use systematic qualitative methods of analysis; instead, they provided short interview segments, using personal judgment in selecting representative quotes.

In another recent qualitative study, Constantine and colleagues (2005) interviewed 16 Asian international college women about their adjustment experiences in the U.S. In terms of ethnic heritage, the women in this study identified as Indian $(n=4)$, Japanese ( $n$ $=4)$, Korean $(n=4)$, and Vietnamese $(n=4)$. Semi-structured interviews were conducted with each participant, and data were analyzed using consensual qualitative research. One of the interview questions elicited information about participants' intentions to seek counseling when coping with the cultural transitions of studying abroad. Participants overwhelmingly indicated that they consider counseling as their last option. For example, a Korean participant stated: "I would speak to a counselor if my family and friends did not have the answer to my problem or if I was feeling bad and did not want them to see me down. I would do this if it were the last option.” (p. 170). Thus, qualitative data indicate that some Asian international women may have doubts about seeking counseling and, thus, have lower intentions to access mental health services. The findings of this study, however, are limited by the fact that intentions to seek counseling were not the major focus of the data collection process. 
Taken together, these studies indicate that Asian international students may report low intentions to seek counseling for psychological problems. However, each of these investigations suffers from important methodological limitations. One problem involves the use of convenience sampling and small sample sizes, which limit the power of statistical analyses (Tabachnick \& Fidell, 2007). Another limitation is the use of modified or unvalidated questionnaires designed for the individual study. Finally, none of the studies examined help-seeking intentions as the primary variable of investigation. Given these limitations, further research needs to examine Asian international students' helpseeking intentions using well-validated assessment instruments. In addition, intentions to seek counseling should be examined in combination with other cognitive precursors of help-seeking behavior, such as counseling attitudes and stigma concerns. The next two sections of this literature review direct attention to attitudes and perceived stigma as two additional cognitive predictors of Asian international students' counseling use.

\section{Attitudes toward Seeking Counseling}

Attitudes have been defined as a cognitive predisposition to "respond to an object in a consistently favorable or unfavorable manner" (Ajzen \& Fishbein, 1980, p. 41). According to Elhai, Schweinle, and Anderson (2008) counseling attitudes are most commonly operationalized through the Attitudes Toward Seeking Professional Psychological Help Scale (ATSPPH; Fischer \& Turner, 1970) and its shorter and psychometrically superior form (ATSPPH-SF; Fischer \& Farina, 1995). The ATSPPH was originally developed as a 29-item multidimensional measure of attitudes toward seeking psychological counseling (Fischer \& Turner, 1970). Exploratory factor analyses with a sample of 424 college students revealed a four-dimensional structure: Factor I - 
recognition of the need for psychological help; Factor II - stigma tolerance; Factor III interpersonal openness; and Factor IV - confidence in the mental health profession. Coefficient alphas for the four subscales, however, were generally low, ranging from $\alpha=$. 62 for emotional openness to $\alpha=.74$ for confidence in the mental health field. In contrast, the internal consistency of total ATSPPH scores was deemed acceptable $(\alpha=.83)$. Given this limitation, Fisher and Turner advised against the use of the individual ATSPPH subscales and recommended the use of total ATSPPH scores instead.

Subsequently, Fischer and Farina (1995) developed the abbreviated and psychometrically superior 10-item ATSPPH-SF scale, drawing primarily from the two factors of recognition of the need for counseling and confidence in the mental health profession, while omitting the stigma tolerance and emotional openness factors. These authors argued that recognizing the need for psychological help and having confidence in the mental health field constitute the core construct of counseling attitudes, and, thus, should be the primary factors included in the revised ATSPPH-SF scale. They further suggested that emotional openness and stigma tolerance may constitute separate psychological variables that correlate with counseling attitudes but do not belong to the content domain of the construct. Consistent with this hypothesis, the internal consistency of the total abbreviated ATSPPH-SF was comparable to that of the original version, $\alpha=$ .84 in a sample of 389 undergraduates, despite its shorter length. Given these conceptual and psychometric improvements, the abbreviated 10-item ATSPPH-SF has been cited as a superior measure as compared to its 29-item predecessor (Elhai, et al., 2008).

Given the availability of the above counseling attitude measures, researchers have gradually begun to examine this construct with samples of Asian international students. 
For example, Tedeschi and Willis (1993) explored attitudes toward seeking counseling in a sample of 66 Asian international and 48 European American college students. The Asian students in the sample came from the following countries of origin: Malaysia $(n=$ $27)$, Taiwan $(n=15)$, Thailand $(n=10)$, Korea $(n=9)$, and China $(n=5)$. Survey respondents completed a demographic data sheet, a rating of preferred counselor characteristics, and the original 29-item ATSPPH scale. Results indicated a significant gender x country of origin interaction in predicting participants' total ATSPPH scores, $F$ $(1,110)=7.58, p<.01$. Specifically, U.S.-born women expressed significantly more positive attitudes $(M=83.81)$ than did U.S.-born men $(M=72.18)$, Asian international women $(M=74.03)$, and Asian international men $(M=72.44)$. Furthermore, U.S.-born college women reported greater need for counseling, greater emotional openness, higher stigma tolerance, and more confidence in the mental health field, as indicated by significantly higher scores on the four ATSPPH subscales than the other three groups. One limitation of this study was the small sample size and low statistical power, which may have made it difficult to find other potentially significant differences between Asian international and American students.

A more recent investigation by Yoon and Jepsen (2008) compensated for this limitation by comparing the counseling attitudes of 189 Asian international and 186 American graduate students. The Asian students represented the following countries of origin: China (48.7\%), Korea (36.5\%), Taiwan (7.9\%), and Japan (6.9\%). All participants completed a demographic data sheet together with a newly developed 17-item measure of counseling attitudes. A factor analysis of the 17-item questionnaire yielded four factors: Factor 1 - lack of self-perceived need for counseling; Factor 2 - discomfort and shame 
with counseling; Factor 3 - openness to counseling; and Factor 4 - preference for a directive counseling style. Results indicated that, compared with American students, Asian international students had significantly less positive counseling attitudes, $F$ (4, $365)=35.68, p<.001, \eta^{2}=.28$. Furthermore, Asian international students indicated less self-perceived need, less emotional openness, and greater discomfort and shame with counseling. Gender differences also emerged; male students revealed less self-perceived need, less emotional openness, and greater shame with counseling than did female students, $F(4,365)=5.12, p<.01, \eta^{2}=.02$. It is notable, however, that the overall effect of country of origin was much larger than that of gender, indicating that Asian international students from either gender had significantly less positive attitudes. A limitation of this study was the utilization of an attitudinal measure developed for the purpose of the study without adequate evidence of its construct validity and reliability.

In another study on international students' counseling attitudes, Dadfar and Friedlander (1982) investigated possible within-group differences among students from different geographic regions. The participants were 172 foreign students who came from Asia $(n=53)$, Latin America $(n=39)$, Europe $(n=36)$, and Africa $(n=36)$, and who completed the original 29-item ATSPPH scale and a demographic data sheet. The total ATSPPH scores of all participants were factor analyzed, yielding three dimensions: Factor 1 - confidence in the mental health field; Factor 2 - concern about counseling stigma; and Factor 3 - preference for dealing with psychological problems on one's own. Between-group analyses indicated that international students from Europe and Latin America had significantly more positive counseling attitudes than did students from Asia or Africa, $F(3,156)=9.33, p<.0001$. Mean total ATSPPH scores for students coming 
from different geographic regions were as follows: Latin America $(M=57.1)$, Europe ( $M$ = 53.9), Africa $(M=46.4)$, and Asia $(M=46.4)$, with higher scores indicating more positive attitudes. A particular strength of this study was the examination of within-group difference along the lines of region of origin. However, one limitation was the authors' failure to examine possible cultural variables as predictors of international students' counseling attitudes.

Komiya and Eells (2001) conducted another recent study examining international students' attitudes toward seeking counseling. The participants of this study were 121 international students coming from Asia $(45 \%, n=55)$, Latin America $(23 \%, n=28)$, Europe $(18 \%, n=22)$, Africa $(6 \%, n=7)$, Canada $(4 \%, n=5)$, the Middle East $(2 \%, n=$ 2), and Russia or the former Soviet Union $(2 \%, n=2)$. Participants completed the ATSPPH-SF, the Emotional Openness Scale, the Hopkins Symptom Checklist, and a demographic questionnaire. A stepwise multiple regression analysis indicated that emotional openness was a significant unique predictor of international students' attitudes toward seeking counseling $\left(\beta=.21, p<.01, R^{2}=.08\right)$, followed by prior counseling experience $\left(\beta=2.73, p<.01, R^{2}=.03\right)$ and participants' sex $\left(\beta=1.70, p<.05, R^{2}=.04\right)$. Specifically, international students who were emotionally open, who had prior counseling experience, and who were female tended to endorse more positive attitudes. A limitation of this study was that the authors failed to examine within-group differences based on participants' country of origin. Similarly, the authors failed to explore possible cultural factors as predictors of participants' attitudes toward seeking counseling.

Zhang and Dixon (2003) conducted one of the few studies examining cultural predictors of Asian international students' counseling attitudes. The participants were 170 
foreign-born Asian students from the following countries: China and Hong Kong (32\%), Korea (17\%), Japan (16\%), India (11\%), Thailand (7\%), Taiwan (5\%), Malaysia (2\%), Indonesia (2\%), and other (9\%). Participants completed a modified form of the SuinnLew Asian Self-Identity Acculturation Scale (SL-ASIA), the original 29-item ATSPPH scale, and a demographic questionnaire. Data were analyzed using multiple regression, with demographic variables (age, gender, country of origin, length of stay in the U.S., and past therapy experience) and SL-ASIA scores predicting participants' total ATSPPH scores. None of the demographic variables emerged as a significant predictor; however, participants' SL-ASIA scores accounted for a unique portion of variance in counseling attitudes, such that more highly acculturated Asian international students held more positive attitudes, $F(1,156)=15.37, p<.01, R^{2}=.09$. In addition, SL-ASIA scores accounted for significant variance in two ATSPPH subscales: stigma tolerance, $F(1,153)$ $=18.40, p<.01, R^{2}=.10$, and confidence in the mental health field, $F(1,153)=8.19, p<$ $.01, R^{2}=.04$. Thus, more highly acculturated Asian international students had greater trust in mental health professionals and were less concerned about the stigma of seeking help. A limitation of the study was the use of a modified version of the SL-ASIA scale, with little empirical evidence to support its psychometric properties.

Finally, Liao and colleagues (2005) examined a set of cultural and cognitive predictors of counseling attitudes, using a sample of 202 Asian or Asian American and 336 European American college students. Of the 202 Asian participants, 133 (66\%) were U.S. citizens or permanent residents, and $69(34 \%)$ were international students. The Asian countries of origin represented in the sample included: China (24.8\%), Korea (17.8\%), Taiwan (10.9\%), the Philippines (8.4\%), India (5.9\%), Japan (2\%), Vietnam 
(1.5\%), Thailand (0.5\%), Cambodia (0.5\%), Iran (0.5\%), Singapore (0.5\%), and other (5.4\%). All participants completed a demographic questionnaire, the Interpersonal Support Evaluation List, the Personal Problem Inventory, the Self-Concealment Scale, and the 29-item ATSPPH scale. In addition, the Asian and Asian American students also completed the SL-ASIA scale and the Asian Values Scale (AVS). A preliminary path model indicated that social support and psychological distress were positively associated, whereas self-concealment was negatively associated, with total ATSPPH scores for both Asian and Asian American $\left(\chi^{2}=9.56, d f=4, \mathrm{RMSEA}=.086, \mathrm{CFI}=.97\right)$ and European American students $\left(\chi^{2}=4.95, d f=4\right.$, RMSEA $=.027$, CFI $\left.=1.00\right)$. Adding SL-ASIA and AVS scores improved model fit for Asian and Asian American students $\left(\chi^{2}=27.07, d f=\right.$ $10, \mathrm{RMSEA}=.096, \mathrm{CFI}=.91)$ as compared to a model constraining the effects of these variables to zero $\left(\chi^{2}=48.63, d f=12, \mathrm{RMSEA}=.130, \mathrm{CFI}=.81\right)$. The findings of this study indicate that acculturation level and adherence to cultural values may predict Asian students' counseling attitudes. A significant limitation was combining foreign-born Asian international and U.S.-born Asian American students in the same sample while ignoring within-group differences such as citizenship status and length of stay in the U.S.

Taken together, this body of literature suggests that Asian international students may hold more negative counseling attitudes as compared to American students (Tedeschi \& Willis, 1993; Yoon \& Jepsen, 2008). This body of literature, however, suffers from some limitations. Several studies used a small sample size, potentially resulting in insufficient statistical power to detect significant within-group and betweengroup differences (Komiya \& Eells, 2001; Tedeschi \& Willis, 1993). Other studies used newly-designed or modified measures, without adequate evidence for their psychometric 
properties (Yoon \& Jepsen, 2008; Zhang \& Dixon, 2003). One study combined foreignborn Asian students with international students from other geographic regions while failing to examine differences based on country of origin (Komiya \& Eells, 2001). Another investigation used a combined sample of foreign-born Asian international and U.S.-born Asian American students without examining possible differences based on citizenship status and length of stay in the U.S. (Liao et al., 2005). Finally, only one study examined both cultural and cognitive predictors of Asian international students' counseling attitudes, using an a priori specified theoretical model (Liao et al., 2005).

Given these methodological limitations, further research needs to examine Asian international students' counseling attitudes using larger and more representative samples, allowing for sufficient statistical power to detect significant results (Tabachnick \& Fidell, 2007). Furthermore, future research should employ well-researched and validated assessment instruments, as well as instruments offering superior psychometric properties, such as the abbreviated ATSPPH-SF. Attention also needs to be directed to Asian international students specifically, rather than combining them with samples of foreignborn students from other regions or with U.S.-born Asian American undergraduates. Finally, future research needs to explore other cognitive predictors of mental health service use, apart from an exclusive focus on counseling attitudes. Specifically, it has been suggested that attitudes may not be the sole predictor of help-seeking intentions and behavior, and other cognitive factors, such as social stigma concerns, may exert an equally important influence on individuals' intentions to seek counseling. 


\section{Stigma Concerns and Seeking Counseling}

The social stigma of seeking counseling has been previously defined as "the perception that a person who seeks psychological treatment is undesirable or socially unacceptable" (Vogel et al., 2006, p. 325). Similarly, Deane and Chamberlain (1994) defined stigma concerns as the fear that significant others will judge a person more negatively if he or she seeks professional psychological care. The social stigma of counseling has been cited as one of the most pervasive barriers to mental health service utilization (Corrigan, 2004; Schomerus \& Angermeyer, 2008; Vogel, Wester, \& Larson, 2007). For example, Corrigan (2004) suggested that, because seeking counseling is socially stigmatized, people who utilize professional mental health care may become victims of labeling, negative stereotypes, prejudice, and discrimination. As a result, individuals may refrain from seeking needed professional psychological help in order to avoid the stigma attached to using mental health services. Similarly, in their narrative review of the empirical literature on stigma and help-seeking, Schomerus and Angermeyer (2008) found overwhelming evidence that anticipated social stigma is significantly associated with a reduced willingness to seek professional psychological services. These authors concluded that the anticipation of being socially stigmatized may deter many individuals from seeking the mental health services they need.

A greater recognition of stigma's impact on help-seeking behavior has recently resulted in several newly developed measures assessing different aspects of counseling stigma. Whereas older conceptualizations tended to view stigma concerns as a subset of help-seeking attitudes (Fisher \& Turner, 1970; Deane \& Chamberlain, 1994), more recent writings have emphasized the conceptual uniqueness of stigma as a construct in its own 
right (Komiya, Good, \& Sherod, 2000; Vogel, Wade, \& Ascheman, 2009). For example, in their revised ATSPPH-SF scale, Fischer and Farina chose to exclude the original stigma tolerance factor, recognizing that stigma concerns are distinct from counseling attitudes. More recently, Komiya and colleagues (2000) developed the Stigma Scale for Receiving Psychological Help (SSRPH), a five-item measure of individuals' perceptions that society stigmatizes mental health service utilization. Similarly, Vogel et al. (2009) validated the 5-item Perceptions of Stigmatization by Others for Seeking Help Scale (PSOSH), a measure assessing fears of being stigmatized by significant others in one's social network. Finally, Vogel and colleagues (2006) validated the 10-item Self-Stigma of Seeking Help Scale (SSOSH), an assessment of internalized self-stigma in the context of counseling. Given these new psychometric developments, the stigma of seeking psychological services is increasingly gaining recognition as an important construct.

Given the greater availability of counseling stigma measures, researchers have begun to examine this construct with Asian international students. Dadfar and Friedlander (1982) examined stigma concerns in a study that was described earlier in this review. All participants completed the original 29-item ATSPPH, which yielded a three-factor solution: Factor 1 - confidence in the mental health field $(\alpha=.65)$; Factor 2 - counseling stigma concerns $(\alpha=.51)$; and Factor 3 - preference for dealing with psychological problems on one's own $(\alpha=.55)$. MANOVA analyses suggested that international students with no prior therapy experience expressed significantly greater stigma concerns than did students who had sought counseling in the past, $F(9,401)=3.39, p=.0006$. These findings indicate that past help-seeking behavior may be significantly associated with Asian international students' counseling stigma concerns. A significant limitation of 
this study was that stigma was conceptualized as a subset of counseling attitudes, rather than as a distinct construct. In addition, the stigma concerns factor identified in this study had low internal consistency, which may be deemed unacceptable (Tabachnik \& Fidell, 2007). Finally, the authors failed to examine the association between stigma and other help-seeking constructs, such as attitudes and intentions to seek counseling.

Tedeschi and Willis (1993) conducted a similar study comparing the counseling attitudes and stigma concerns of Asian international and European American college students. Participant demographics and study design were reported earlier in this literature review and, thus, will not be repeated here. All respondents completed the original 29-item ATSPPH scale, which included the initial 5-item counseling stigma tolerance subscale. ANOVA analyses indicated a significant interactive effect for gender and country of origin in predicting participants' stigma concerns. U.S.-born college women were significantly less concerned with the social stigma of seeking psychological counseling, $F(1,110)=4.45, p<.05$, as compared to U.S.-born men and foreign-born men and women. Specifically, European American women $(M=16.77)$ were significantly more tolerant of counseling stigma than were European American men $(M=$ 14.46), Asian international women $(M=14.57)$, and Asian international men $(M=$ 14.08). These findings are limited, however, by the fact that the authors measured stigma concerns as a subset of help-seeking attitudes rather than as a construct in its own right. In addition, these authors failed to examine possible relationships between the stigma tolerance factor and participants' counseling attitudes and help-seeking intentions.

Zhang and Dixon (2003) built on previous research by examining the relationship between cultural factors and Asian international students' stigma concerns. The sample 
characteristics and study procedures were reported earlier and, thus, will not be repeated here. All participants completed a demographic data sheet, a modified form of the SLASIA scale, and the original 29-item ATSPPH scale. Data were analyzed using four regression equations, with demographic variables (age, gender, country of origin, length of stay in the U.S., and past therapy experience) and SL-ASIA scores entered as predictors of the four ATSPPH subscales (i.e., recognition of the need for counseling, emotional openness, stigma tolerance, and confidence in the mental health field). None of the demographic factors explained a significant amount of variance in stigma tolerance; however, SL-ASIA scores emerged as a significant, unique predictor, accounting for $10 \%$ of the variance in the stigma subscale, $F(15,153)=2.344, \beta=.334, p<.01$. As expected, more highly acculturated Asian international students showed greater tolerance toward counseling stigma. Similar to previous studies, however, this investigation conceptualized stigma as a subset of help-seeking attitudes. In addition, the authors failed to examine possible associations between stigma concerns and other help-seeking variables, such as attitudes, intentions, and behavior.

Finally, a more recent study by Yoon and Jepsen (2008) compared 189 Asian international and 186 U.S. graduate students in terms of discomfort and shame with counseling. The characteristics of the sample and study procedures were discussed earlier in this review. Participants completed a demographic questionnaire and a 17-item measure of counseling attitudes that yielded the following dimensions: Factor $1-$ perceived need for counseling; Factor 2 - discomfort and shame with counseling; Factor 3 - openness to counseling; and Factor 4-preference for a directive counseling style. Data indicated that, compared to U.S. students, Asian students experienced greater shame 
with seeking counseling, $F(4,365)=35.68, p<.001, \eta^{2}=.28$. Gender differences emerged, such that men reported greater shame than women, $F(4,365)=5.12, p<.01$, $\eta^{2}=.02$. In general, U.S.-born women reported the least shame $(M=2.10, S D=.51)$, followed by U.S.-born men $(M=2.28, S D=.60)$, Asian women $(M=2.31, S D=.51)$, and Asian men $(M=2.41, S D=.55)$. A limitation of this study was using an unvalidated counseling attitudes measure, as well as failing to examine stigma concerns as a construct in its own right. In addition, the shame and discomfort factor identified by these authors may not have fully overlapped with stigma concerns, as conceptualized in other research.

In conclusion, I was able to identify only four studies examining counseling stigma concerns among Asian international students. Each of these studies suffered from conceptual and methodological limitations. In general, researchers measured stigma as a subset of counseling attitudes, rather than as a construct in its own right. This approach, however, is at odds with recent theoretical developments that have emphasized the conceptual uniqueness of stigma concerns. In addition, none of the studies examined stigma in relation to other cognitive predictors of counseling service utilization, such as help-seeking attitudes and intentions. Finally, three studies (Dadfar \& Friedlander, 1982, Tedeschi \& Willis, 1993; Yoon \& Jepsen, 2008) examined only demographic predictors (e.g., age, gender, length of stay in the U.S., and past therapy experience) in relation to stigma, while failing to consider potential cultural predictors of stigma concerns. In fact, only one study (Zhang \& Dixon, 2003) examined a cultural factor, acculturation, in predicting Asian international students' counseling stigma concerns.

Given these limitations, further research needs to examine Asian international students' perceptions that seeking counseling carries social stigma. Such research needs 
to conceptualize stigma concerns as a construct in its own right, as well as use the theoretically grounded, psychometrically sound stigma measures that have recently been developed. Furthermore, future research on Asian international students' help-seeking may benefit from examining the relationship between different help-seeking constructs (i.e., intentions, attitudes, and stigma concerns) using a theoretically-grounded model. Finally, research needs to examine the relationship between these help-seeking variables and different cultural predictors of Asian international students' utilization of counseling services. Three such cultural variables are acculturation, Asian values, and concerns with public loss of face. The next three sections of this literature review will discuss each of these cultural factors in relation to Asian international students' counseling use.

\section{Acculturation and Seeking Counseling}

Acculturation has been defined as a "culture change that results from continuous, first hand contact between two distinct cultural groups" (Berry, 1994, p. 129). Similarly, Suinn and Khoo (1995) defined acculturation as "the process that can occur when two or more cultures interact" (p. 139). Although acculturation was initially conceptualized as a group-level phenomenon (Berry, 1994; Suinn \& Khoo, 1995), more recent theoretical and empirical writings have examined this construct as an individual difference factor (Berry, 2003; Kim \& Abreu, 2001; Miller, 2007). At an individual level, acculturation has often been defined as the process of psychological, behavioral, and attitudinal change that occurs when an individual is exposed to a second culture (Kim \& Abreu, 2001). Such changes may occur across distinct psychological and behavioral domains, including cultural knowledge, identity, ethnic interaction and pride, participation in ethnic traditions and rituals, and preferred language use. Given the complexity of this construct, 
it is not surprising that psychologists are still debating different conceptualization and measurement approaches (Chung, Kim, \& Abreu, 2004; Kim \& Abreu, 2001). Initial theorizing and measurement in the area of Asian and Asian American acculturation emphasized a unidimensional construct indicating a progressive loss of one's native identity accompanied by growing identification with the host culture (Suinn, Ahuna, \& Khoo, 1992; Suinn, Rickard-Figueroa, \& Vigil, 1987). According to this model, acculturation occurs on a single continuum, such that an increasing association with American culture necessitates progressive loss of one's ties with the Asian community. Thus, the unidimensional approach suggests that, as Asian individuals acculturate to American language, customs, and social norms, they will progressively lose their Asian identity and become assimilated into the host society (Suinn et al., 1992). A unidimensional measurement of acculturation typically provides researchers with a single acculturation score, such that a low ranking indicates endorsement of Asian norms and traditions, whereas a high ranking demonstrates loss of native cultural identity and an acquisition of American norms (Suinn et al., 1992; Suinn et al., 1987).

More recently, the unidimensional view of acculturation has come under attack because of its assumption that individuals need to abandon their native heritages in order to acculturate to the host society (Chung et al., 2004; Kim \& Abreu, 2001; Miller, 2007). Kim and Abreu, for example, argued that immigrants may be able to maintain their ties to their culture of origin, while at the same time acquiring the norms, values, and behaviors of the host society. In other words, these authors suggested that the maintenance of one's native heritage and the acquisition of new cultural knowledge are not mutually exclusive, but could occur in parallel in the same individual. This hypothesis has, in fact, been 
supported by empirical findings. Using a sample of 342 Asian American undergraduates, Chung and colleagues (2004) found that participants' identification with their Asian heritage was essentially independent from their acculturation to American society $(r=$ $.06, \mathrm{p}>.05$ ). Similarly, Miller (2007) used structural equation modeling to examine the hypothesized independence of native-culture vs. host-culture identification in a sample of 288 Asian American college students. The analysis indicated a superior model fit when participants' identifications with their native heritage and with American society were modeled as two separate dimensions. Thus, empirical evidence indicates that the unidimensional view may not reflect the actual complexity of the acculturation process. Given these problems with the original unidimensional assessment of acculturation, a newer, bidimensional approach has been increasingly gaining attention (Chung et al., 2004; Kim \& Abreu, 2001). In contrast to the unidimensional approach, a bidimensional conceptualization suggests that individuals' identification with the native and host cultures occurs on two separate, orthogonal continua (Chung et al., 2004; Kim \& Abreu, 2001; Miller, 2007). One continuum, referred to as "enculturation," assesses degree of identification with one's native customs and traditions. A second continuum, called "acculturation" measures level of identification with the host society. The conceptual advantage of this approach is that it allows individuals to retain a native identity, while at the same time acquiring the behaviors and norms of the host culture. The advantage of the bidimensional approach is that it allows for the development of a bicultural identity, whereby individuals are able to integrate aspects of both native and host identifications (Kim \& Abreu, 2001). A bidimensional measurement of enculturation 
and acculturation will then provide two separate scores, each assessing individuals' identification with their native or host cultures, respectively (Chung et al., 2004).

These two conceptual approaches have resulted in two distinct paradigms for assessing Asian and Asian American acculturation. The older, unidimensional approach is most often measured by the Suinn-Lew Asian Self-Identity Acculturation Scale (SLASIA; Suinn et al., 1992; Suinn et al., 1987). SL-ASIA is a 21-item, multiple choice measure assessing the following acculturation domains: language preferences (4 items), cultural identification (4 items), friendship choice (4 items), participation in ethnic traditions (5 items), generation and geographic history ( 3 items), and cultural attitudes (1 item). As recommended by Suinn et al. (1997) participants' responses are summed to yield a single total acculturation score, denoting one's acculturation to American society. Separate analyses by topic domain are not commonly conducted because of the small number of items comprising each subscale. Consistent with a unidimensional conceptualization, low SL-ASIA scores indicate strong Asian orientation, whereas high SL-ASIA scores indicate strong American identification (Suinn et al., 1987; Suinn et al., 1992). The scale has demonstrated good psychometric properties $(\alpha=.88-.91$ for total SL-ASIA scores) with three samples of Asian American college students (Suinn et al., 1987; Suinn et al., 1992). Since its creation, the measure has been used extensively with Asian American populations and has been found to relate in expected directions with participants' generation status, length of residence in the U.S., and self-ratings of cultural identity (Suinn et al., 1987). In fact, to date, most of the literature on Asian and Asian Americans' acculturation has been conducted using the SL-ASIA (Suinn et al., 1992). 
However, a s limitation of the SL-ASIA is the assumption that Asian and American cultural identifications occur on a single continuum and, thus, are mutually exclusive.

More recently, Chung and colleagues (2004) created the Asian American Multidimensional Acculturation Scale (AAMAS), a measure using a bidimensional conceptualization of the acculturation process. To date, this is the only bidimensional acculturation measure designed specifically for Asian and Asian American populations (Chung et al., 2004). The AAMAS is a 30 -item measure consisting of two subscales: AAMAS-CO, assessing identification with the culture of origin (i.e., enculturation), and AAMAS-EA, assessing identification with European American culture (i.e., acculturation). Each subscale consists of 15 items measuring the domains of cultural identity (6 items), cultural knowledge ( 3 items), language preferences (4 items), and food consumption ( 2 items). The items are summed across domains, to yield a single subscale score; separate analyses per domain are not typically conducted, given the small number of items under each domain. A high AAMAS-CO score indicates strong identification with Asian culture (i.e., enculturation), whereas a high AAMAS-EA score indicates strong identification with European American society (i.e., acculturation). Consistent with a bidimensional conceptualization of acculturation, the AAMAS-CO and the AAMAS-EA subscales were not significantly correlated $(r=-.06, p>.05)$ in a sample of 342 Asian American college students. Furthermore, both scales demonstrated good psychometric properties $(\alpha=.76-.81$ for the AAMAS-CO; $\alpha=.76-.81$ for the AAMASEA) with three samples of Asian American undergraduates. Finally, the two AAMAS subscales related in predicted directions with participants' generation status, SL-ASIA scores, and endorsement of Asian values. Thus, the AAMAS has emerged as a promising 
new scale of bidimensional acculturation. However, probably because of its novelty and more complex bidimensional structure, the AAMAS has not yet been used extensively in existing research, highlighting the need to employ this measure in future studies.

Given the available acculturation assessment instruments, researchers have begun to examine this construct in relation to Asian and Asian Americans' help-seeking attitudes, intentions, and behavior (Atkinson \& Gim, 1989; Liao et al., 2005; Tata \& Leung, 1994; Ting \& Hwang, 2009). Unfortunately, most of this literature has focused on U.S.-born Asian Americans who identified as either U.S. citizens or permanent residents. Much less attention has been directed to the relationship between acculturation and helpseeking among foreign-born Asian international students. In fact, I was able to locate only two studies which specifically examined the relationships among acculturation, counseling attitudes, and help-seeking behavior using samples of Asian international students (Frey \& Roysircar, 2006; Zhang \& Dixon, 2003). Given the scarce empirical literature in this topic domain, I will first review existing studies of Asian American undergraduate samples, followed by a discussion of the two investigations which specifically focused on Asian international students' acculturation and help-seeking. Atkinson and Gim (1989) examined acculturation as a predictor of counseling attitudes in an Asian and Asian American college student sample $(N=557)$. Participants identified as Chinese American $(n=263)$, Japanese American $(n=185)$, and Korean American $(n=109)$, with all respondents self-identifying as U.S. citizens or permanent residents. Participants completed a demographic questionnaire, the 21-item SL-ASIA, and the original 29-item ATSPPH scale. Based on SL-ASIA scores, participants were divided into low, medium, and highly acculturated subgroups. Subsequently, a one-way 
MANOVA was performed using the four ATSPPH subscales (i.e., perceived need for counseling, stigma tolerance, emotional openness, and confidence in the mental health field) as outcome measures. The model was significant, $F(8,1084)=3.476, p<.001$, with subsequent univariate analyses indicating significant effects for three of the ATSPPH subscales: stigma tolerance, $F(2,545)=3.978, p=.019$; need for counseling, $F(2,545)=4.371, p=.013$; and emotional openness $F(2,545)=10.992, p<.001$. Specifically, the more highly acculturated Asian American students were more likely to recognize the need for counseling, to be tolerant of counseling stigma, and to value emotional openness. A limitation of this study was the use of the SL-ASIA as a unidimensional acculturation measure, which is at odds with current theorizing.

In a similar study, Tata and Leung (1994) examined acculturation as a predictor of counseling attitudes in a sample of 210 Chinese American students. These authors did not collect information about participants' U.S. citizenship and immigration status, so it is unclear whether the sample included any international students. Participants completed a demographic questionnaire, the 21-item SL-ASIA, and the original 29-item ATSPPH scale. Bivariate correlations revealed a positive association between participants' SLASIA and total ATSPPH scores $(r=.16, p<.05)$. Subsequently, a hierarchical multiple regression analysis was conducted with gender and SL-ASIA scores entered as predictors of participants' total ATSPPH scores. Both gender $\left(\beta=-.15, p<.05, R^{2}=.02\right)$ and acculturation $\left(\beta=.16, p<.05, R^{2}=.03\right)$ emerged as significant unique predictors of counseling attitudes. Specifically, Chinese American students who were female and more highly acculturated endorsed more positive counseling attitudes. However, the findings of this study are limited by the use of the SL-ASIA as a unidimensional acculturation 
measure, such that participants' identification with the Asian and American cultures are seen as mutually exclusive and incompatible.

More recently, Ting and Hwang (2009) extended the previous two studies by utilizing a multiethnic, bidimensional acculturation scale as a predictor of Asian American students' counseling attitudes. The participants of this study were 107 Asian American college students, all of whom identified as U.S. citizens or permanent residents. The five largest ethnic groups in the sample were Chinese (33\%), Vietnamese (19\%), Japanese (14\%), Taiwanese (11\%), and Korean (9\%). Participants completed a demographic questionnaire, the Vancouver Index of Acculturation (VIA, a multi-ethnic bidimensional acculturation scale assessing heritage and mainstream cultural identification), and the abbreviated 10-item ATSPPH scale. Contrary to expectations, the two VIA dimensions failed to predict participants' counseling attitudes. Neither heritage cultural identification $(r=.00, p>.05)$, nor mainstream cultural identification $(r=.05, p$ $>$.05) emerged as significant predictors of ATSPPH scores. A possible explanation for these null findings is the use of the VIA, a multi-ethnic measure that may not be sensitive to the acculturation experiences of Asian Americans. In fact, the newly developed AAMAS may have been more appropriate for testing the research question in this study, given its bidimensional format and its focus on Asian and Asian American populations. Apart from examining counseling attitudes, researchers also have investigated intentions to seek counseling in relation to acculturation. Atkinson, Lowe, and Matthews (1995) examined gender, acculturation, and prior therapy experience as predictors of Asian American students' intentions to seek counseling $(N=187)$. All participants were either U.S. citizens or permanent residents and identified as Chinese American $(n=15)$, 
Japanese American $(n=33)$, Korean American $(n=44)$, Filipino American $(n=39)$, Southeast Asian American $(n=18)$, or Pacific Islander $(n=38)$. Participants completed a demographic data sheet, the SL-ASIA, and a single item assessing intentions to seek counseling for a personal problem. Participants' SL-ASIA scores were used to separate the sample into low, medium, and highly acculturated groups. Then, an ANCOVA was conducted with two group factors (gender and acculturation) and one covariate (previous therapy experience) as predictors of intentions to seek counseling for a personal problem. Results indicated no main or interaction effects for either gender, $F(1,465)=2.94, p<$ .05 , or acculturation, $F(2,465)=.06, p<.05$. However, prior counseling experience was a significant predictor, $F(1,465)=43.46, p<.05$, such that participants with past therapy experience reported greater intentions to seek counseling in the future. Two limitations of this study were the use of the unidimensional SL-ASIA as well as employing a single item to assess participants' intentions to seek counseling for personal difficulties.

In a more recent study, Liao and colleagues (2005) examined acculturation as a predictor of Asian and Asian American students' $(N=202)$ counseling attitudes and intentions. The demographic breakdown of the sample was reported earlier in this review. Participants completed a demographic data sheet, measures of social support, psychological distress, and self-concealment, the 29-item ATSPPH scale, and the SLASIA. A path model indicated that social support, psychological distress, and selfconcealment each predicted participants' help-seeking attitudes and intentions $\left(\chi^{2}=9.56\right.$, $d f=4$, RMSEA $=.086$, CFI $=.97)$. Adding SL-ASIA scores improved model fit $\left(\chi^{2}=\right.$ 27.07, $d f=10, \mathrm{RMSEA}=.096, \mathrm{CFI}=.91)$ as compared to a model constraining this effect to zero $\left(\chi^{2}=48.63, d f=12\right.$, RMSEA $\left.=.130, \mathrm{CFI}=.81\right)$. Specifically, SL-ASIA was a 
significant predictor of total ATSPPH scores $(\beta=.13, p<.05)$, such that highly acculturated participants indicated more positive counseling attitudes. Further analyses indicated that SL-ASIA scores influenced participants' help-seeking intentions indirectly through their effect on counseling attitudes. Two limitations of this study were the use of a unidimensional acculturation measure, together with combining foreign-born Asian and U.S.-born Asian American students in the same sample.

Zhang and Dixon (2003) conducted one of the two studies examining acculturation and counseling attitudes in a sample of Asian international students. The demographic characteristics of the sample were reported earlier in this review and, thus, will be omitted here. Participants completed a modified form of the SL-ASIA, the original ATSPPH scale, and a demographic data sheet. Data were analyzed using multiple regression, with demographic variables (age, gender, country of origin, length of stay in the U.S., and past therapy experience) and SL-ASIA entered as predictors of total ATSPPH scores. None of the demographic variables was a significant predictor; however, participants' SL-ASIA scores uniquely predicted counseling attitudes, such that more highly acculturated Asian international students held more positive attitudes, $F(1$, $156)=15.37, p<.01, R^{2}=.09$. In addition, the SL-ASIA significantly predicted scores on two of the ATSPPH factors: stigma tolerance, $F(1,153)=18.40, p<.01, R^{2}=.10$, and confidence in the mental health field, $F(1,153)=8.19, p<.01, R^{2}=.04$. Specifically, more highly acculturated Asian international students had greater confidence in the mental health field and attached less stigma to seeking counseling than those who are less acculturated. A limitation of the study was the use of the unidimensional SL-ASIA 
acculturation scale, thus failing to examine international students' identification with their native cultures as separate from the development of an American identity.

Frey and Roysircar (2006) conducted the only other study examining Asian international students' acculturation and help-seeking. The sample consisted of Asian international students $(N=110)$, with 52\% coming from South Asia (e.g., India, Pakistan, and Sri Lanka) and 48\% coming from East Asia (e.g., China, Taiwan, and Hong Kong). Participants completed a demographic questionnaire, the American-International Relations Survey (AIRS, measuring adaptation to the U.S.), and an 18-item help-seeking questionnaire developed for the purpose of the study. The 18-item help-seeking questionnaire asked participants to indicate their frequency of using the following help resources: Non-Western spirituality, structured problem-solving, social support, and psychological services. A hierarchical multiple regression was conducted, with acculturation entered as predictors of help resource utilization. Results indicated that acculturation $\left(\beta=-.24, p<.01, R^{2}=.24\right)$ emerged as a significant unique predictor of help resource utilization $\left(\beta=.35, p<.001, R^{2}=.14\right)$, such that more highly acculturated participants reported greater help-resource utilization. A significant limitation of this study was that the authors collapsed participants' ratings of different help resources into a single score, thus obscuring differences between formal vs. informal help-seeking.

Taken together, this literature suggests that acculturation may be an important cultural predictor of Asian and Asian American students' counseling attitudes and intentions (Atkinson \& Gim, 1989; Atkinson et al., 1995; Liao et al., 2005; Tata \& Leong, 1994). However, these investigations suffer from a number of conceptual and methodological limitations. Most of the studies used a unidimensional acculturation 
measure, the SL-ASIA, while ignoring current theoretical and empirical advances, such as the development of the AAMAS (Chung et al., 2004). Furthermore, most of the studies reviewed above surveyed Asian American students who were U.S. citizens or permanent residents, thus excluding Asian international students. One study combined Asian international and Asian American students, thus obscuring possible differences due to immigration status and length of stay in the U.S. (Liao et al., 2005). In fact, only two studies examined acculturation, counseling attitudes, and help-seeking behavior among Asian international students specifically (Frey \& Roysicar, 2006; Zhang \& Dixon, 2003). Finally, few of the studies reviewed above examined acculturation in relation to other important cultural factors, such as Asian values and loss of face concerns.

Given the methodological limitations discussed above, future research should examine the newly designed bidimensional acculturation measure, the AAMAS, as a predictor of Asian international students' help-seeking intentions, attitudes, and stigma concerns. The existing empirical literature can also be advanced by studying acculturation and help-seeking among Asian international students specifically, as opposed to combining foreign-born Asian and U.S.-born Asian American participants in one sample. Finally, future investigations need to examine acculturation in combination with other cultural factors that may be relevant to Asian international students. One such variable that may be especially applicable to this group of students is adherence to traditional Asian cultural values, such as collectivism, filial piety, and humility.

\section{Asian Values and Seeking Counseling}

Scholars in the multicultural counseling field have long emphasized the existence of distinct value orientations characterizing European American and Asian cultures (Kim, 
Atkinson, \& Yang, 1999; Leong \& Chou, 1996; Pedersen, 1991; Sue \& Sue, 2008; Wolfe, Yang, Wong, \& Atkinson, 2001). Specifically, European American culture tends to emphasize autonomy, individualism, individual-oriented decision making, environmental mastery, and future time orientation (Sue \& Sue, 2008; Wolfe et al., 2001). In addition, mainstream American culture values characteristics such as competitiveness, interpersonal directness, emotional openness, and egalitarian social norms (Wolfe et al., 2001). In contrast, Asian cultures often emphasize a distinct set of value orientations such as interdependence, collectivism, group-oriented decision making, and harmony with the environment (Sue \& Sue, 2008; Leong \& Chou, 1996). In addition, Asian cultures value personal characteristics such as humility, self-effacement, emotional self-control, deference to authority figures, filial piety, and conformity to family and social norms (Kim et al., 1999). These distinct value orientations have been hypothesized to affect a wide array of social behaviors, including help-seeking and mental health service utilization (Atkinson et al., 1995; Sue \& Sue, 2008).

In order to facilitate research on cultural values, Kim and colleagues (1999) created the Asian Values Scale (AVS), a 36-item questionnaire assessing the following value orientations: collectivism ( $\alpha=.55)$; conformity to social norms $(\alpha=.77)$; emotional self-control $(\alpha=.52)$; family recognition through achievement $(\alpha=.72)$; filial piety $(\alpha=$ $.44)$; and personal humility $(\alpha=.55)$. In contrast to the individual subscales described above, total AVS scores had good internal consistency $(\alpha=.82)$ and test-retest reliability over a two week period $(r=.83)$, indicating support for the use of the full AVS in research. Furthermore, in support of the scale's construct validity, Kim and colleagues found that AVS items successfully differentiated between first-generation Asian 
Americans $(n=83)$ and U.S.-born European Americans $(n=63)$, such that Asian participants endorsed each value item significantly more strongly than did their European counterparts. AVS total scores also were related in the expected direction to conceptually similar measures, such as the SL-ASIA and the Individualism-Collectivism Scale (INDOCOL). It is important to note that although other scales have been created to measure collectivism-individualism and independent-interdependent self-construal, the AVS is the first scale to assess Asian values specifically (Kim et al., 1999).

More recently, $\mathrm{Kim}, \mathrm{Li}$, and $\mathrm{Ng}$ (2005) sought to improve the internal consistency estimates of the individual AVS subscales by creating the Asian American Values Scale - Multidimensional (AAVS-M). The AAVS-M is a 42-item measure assessing the following internally consistent subscales: Factor I - collectivism $(\alpha=.80)$; Factor II conformity to norms $(\alpha=.79)$; Factor III - emotional self-control $(\alpha=.80)$; Factor IV family recognition through achievement $(\alpha=.90)$; and Factor $\mathrm{V}-$ humility $(\alpha=.81)$. The sixth factor of filial piety that was initially identified in the AVS by Kim and colleagues (1999) was not reliably supported in subsequent analyses, and, thus, was omitted from the AAVS-M. Kim and colleagues noted that the final AAVS-M subscales had higher internal consistency estimates that the original AVS subscales, demonstrating the psychometric superiority of the revised measure. Total AAVS-M scores also showed good psychometric properties, such as high internal consistency $(\alpha=.89)$ and test-retest reliability over a two-week period $(r=.92)$ in an Asian American college sample. Furthermore, total AAVS-M scores correlated significantly and in the expected directions with conceptually related variables, such as interdependent and independent selfconstrual, acculturation, and loss of face concerns. Finally, total AAVS-M scores did not 
correlated with conceptually unrelated constructs, such as self-esteem and social desirability. Thus, Kim et al. (2005) concluded that the AAVS-M offers a psychometrically superior measurement of Asian cultural values and, thus, could be used in research both as individual subscale factors and as a total scale score.

Given the availability of these psychometrically sound measures, several researchers have examined Asian values in relation to counseling attitudes, stigma concern, and help-seeking intentions (Gloria, Castellanos, Park, \& Kim, 2008; Kim, 2007; Kim \& Omizo, 2003; Liao et al., 2005; Shea \& Yeh, 2008; Zayco, 2009). Unfortunately, most of this research has focused on U.S.-born Asian American undergraduates, as opposed to foreign-born Asian international students. Another trend has been to combine foreign-born Asian international students and U.S.-born Asian American students in one sample, without exploring possible within-group differences based on citizenship (Liao et al., 2005; Zayco, 2009). In fact, I could not locate any studies of Asian values and help-seeking among international students specifically. Given this scarcity of research, I will review studies conducted on Asian American samples, reporting, when possible, if the sample included any Asian international students as well. In one recent study, Kim (2007) explored Asian and European American values as predictors of Asian American students' $(N=146)$ counseling attitudes. Participants represented the following nationalities: Chinese (23.3\%), Indian (18.5\%), Korean (9.6\%), Filipino (7.5\%), Japanese (4.8\%), Taiwanese (2.7\%), Vietnamese (2.1\%), Indonesian (1.0\%), Cambodian (1.0\%), Malaysian (1.0\%), Pakistani (1.0\%), Tai (1.0\%), and other $(10.2 \%)$. The authors failed to report participants' citizenship status, leaving it unclear if any international students were included in the sample. Respondents completed a 
demographic questionnaire, the original AVS, the European American Values Scale for Asian Americans (EAVS-AA), and the ATSPPH-SF scale. Bivariate correlations revealed that total AVS scores predicted counseling attitudes $(r=-.24, p<.01)$, as did three of the AVS dimensions: collectivism $(r=-.21, p<.01)$, emotional self-control ( $r=$ $-.22, p<.01)$, and humility $(r=-.17, p<.05)$. In contrast, adherence to European American values did not significantly predict ATSPPH-SF scores $(r=-.05, \mathrm{p}>.05)$. Hierarchical multiple regression indicated that AVS scores predicted counseling attitudes over and above prior therapy experience $\left(\beta=-.24, p<.01, \Delta R^{2}=.06\right)$. A significant limitation of this study was the use of the original AVS rather than the psychometrically superior AAVS-M. In addition, it is unclear whether the results of this study generalize to Asian international students.

In a similar study, Gloria and colleagues (2008) examined Asian values as a predictor of counseling attitudes in a sample of 228 Korean American college students. The authors failed to report respondents' citizenship status, leaving it unclear if any international students were included in the sample. Respondents completed a demographic questionnaire, the original AVS, and the ATSPPH-SF scale. Two hierarchical multiple regressions were conducted (one for each gender) with previous counseling experience and Asian values entered as independent predictors of ATSPPHSF scores. For Korean American women, Asian values emerged as a significant predictor of counseling attitudes ( $\beta=-.28, p<.01, \Delta R^{2}=.08$ ), accounting for $8 \%$ of unique variance over and above prior counseling experience. Specifically, Korean American women who adhered more closely to Asian values also reported more negative counseling attitudes. For Korean American men, however, the effect of Asian values 
failed to reach statistical significance $\left(\beta=-.15, p>.05, \Delta R^{2}=.02\right)$, although the observed relationship was in the hypothesized inverse direction. A significant limitation of this study was the use of the AVS rather than the psychometrically improved AAVS-M. In addition, it is unclear whether the sample included any Korean international students, thus limiting the generalizability of the present results.

More recently, Shea and Yeh (2008) examined Asian values, stigma concerns, and gender as predictors of counseling attitudes among Asian American college students $(N=219)$. Participants' ethnic backgrounds included: Chinese $(45.7 \%)$, Korean $(24.7 \%)$, Japanese (8.7\%), Taiwanese (7.3\%), Indian (4.6\%), Vietnamese (1.8\%), Filipino (1\%), and other (6.4\%). Part of the sample included Asian international students; however, the authors did not report the precise number of foreign participants. Participants completed a demographic questionnaire, the original AVS, the Stigma Scale for Receiving Psychological Help (SSRPH), and the ATSPPH-SF. Bivariate correlations indicated significant associations between AVS scores and help-seeking attitudes $(r=-.24, p<.01)$, and between AVS scores and stigma concerns $(r=.36, p<.01)$. In addition, hierarchical multiple regression indicated that AVS scores were the best unique predictor of helpseeking attitudes $(\beta=-.19, p<.01)$, followed by gender $(\beta=-.15, p<.01)$ and stigma concerns $(\beta=-.14, p<.01)$. In general, participants who were male, endorsed more traditional Asian values, and were concerned with the stigma of counseling expressed less positive counseling attitudes toward seeking psychological help. A limitation of this study was using the original 36-item AVS, rather than the improved AAVS-M, as well as failing to report the exact number of international students included in the sample. 
In another study, Miville and Constantine (2007) examined cultural values and stigma concerns as predictors of Asian American women's intentions to seek counseling. The 201 participants represented the following ethnic backgrounds: Korean (26.4\%), Chinese (23.4\%), Japanese (22.4\%), Taiwanese (15.4\%), Indian (9.0\%), and Vietnamese (3.5\%). All participants were born and reared in the U.S., suggesting that international students were excluded from the sample. Respondents completed a demographic questionnaire, the AVS, the Stigma Scale for Receiving Psychological Help (SSRPH), and the Intentions to Seek Counseling Inventory (ISCI). Bivariate correlations revealed significant associations between total AVS scores and stigma concerns $(r=.19, p<.01)$, and between total AVS scores and intentions to seek counseling $(r=-.17, p<.05)$. Entering stigma in a hierarchical multiple regression significantly reduced the association between total AVS scores and intentions to seek counseling (from $\beta=-.17$ to $\beta=-.13$ ), indicating partial mediation $(\Delta \beta=.04$; Goodman's test statistic $=-1.96, p=.05)$. Thus, it appears that Asian American college women who endorsed more traditional Asian values also reported greater stigma concerns, leading to lower intentions to seek counseling. The results of this study, however, are limited by the use of the original AVS. Furthermore, these findings may not fully generalize to Asian international college women.

Kim and Omizo (2003) conducted another study examining Asian values, counseling attitudes, and help-seeking intentions. The sample included 242 Asian American college students of the following ethnic backgrounds: Chinese (28.1\%), Korean (25.6\%), Filipino (13.2\%), Japanese (11.2\%), Indian (4.1\%), Vietnamese (3.7\%), and other $(6.6 \%)$. It is unclear whether the sample included international students, because the authors did not report participants' citizenship status. Participants completed 
a demographic questionnaire, the AVS, the ATSPPH-SF, and the Willingness to Seek Counseling Questionnaire (WSC, a measure of help-seeking intentions). Bivariate correlations revealed significant negative associations between AVS scores and counseling attitudes $(r=-.39, p<.001)$, and between AVS scores and help-seeking intentions $(r=-.18 ; p<.01)$. Hierarchical multiple regression indicated that AVS scores predicted counseling attitudes after controlling for age, gender, generation status, and past counseling experience $\left(\beta=-.25, p<.001, \Delta R^{2}=.05\right)$. Similarly, AVS scores predicted intentions to seek counseling over and above the demographic factors $(\beta=-.13, p<.05$, $\left.\Delta R^{2}=.01\right)$. Finally, ATSPPH-SF scores fully mediated the relationship between Asian values and help-seeking intentions $(\Delta \beta=.15, p<.001)$. It is unknown, however, whether the results of this study generalize to Asian international students, as these students may not have been represented in the sample.

Whereas the previous studies focused exclusively on Asian American participants, Liao and colleagues (2005) used a combined Asian international and Asian American sample $(N=202)$ to examine cultural values, counseling attitudes, and helpseeking intentions. The demographic composition of the sample was reported earlier in this review and will not be repeated here. Participants completed a demographic data sheet and measures of social support, psychological distress, self-concealment, the 29item ATSPPH scale, the SL-ASIA, and the AVS. A path model indicated that social support, psychological distress, and self-concealment each predicted Asian and Asian American students' help-seeking attitudes and intentions $\left(\chi^{2}=9.56, d f=4\right.$, RMSEA $=$ $.086, \mathrm{CFI}=.97)$. Adding AVS scores improved model fit $\left(\chi^{2}=27.07, d f=10, \mathrm{RMSEA}=\right.$ $.096, \mathrm{CFI}=.91)$ as compared to a model constraining this effect to zero $\left(\chi^{2}=48.63, d f=\right.$ 
12 , RMSEA $=.130, \mathrm{CFI}=.81$ ). In this model, AVS emerged as a significant predictor of total ATSPPH scores $(\beta=-.26, p<.01)$, such that participants who endorsed more traditional Asian values reported less positive counseling attitudes. Further analyses indicated that AVS scores influenced participants' help-seeking intentions indirectly through their effect on counseling attitudes. Two limitations of this study were using the original AVS and combining Asian international and Asian American students.

Finally, Zayco (2009) conducted the only other study which used a combined Asian American and Asian international sample $(N=199)$ in order to examine cultural values and counseling attitudes. The participants represented the following ethnicities: Filipino (24\%), Chinese (21\%), Asian Indian (14\%), Korean (12\%), Taiwanese (8\%), Vietnamese (6\%), Japanese (2\%), and other (13\%). Most respondent (94\%) were U.S. citizens or permanent residents, with a small minority (6\%) identifying as Asian international students. Participants completed a demographic questionnaire, the AAVSM, the Loss of Face scale (LOF), and the original 29-item ATSPPH scale. Bivariate correlations indicated that the five AAVS-M subscales correlated significantly and negatively with total ATSPPH scores: collectivism $(r=-.31, p<.01)$, conformity to social norms $(r=-.31, p<.01)$, emotional self-control $(r=-.55, p<.01)$, family recognition through success $(r=-.26, p<.01)$, and humility $(r=-.15, p<.05)$. A hierarchical multiple regression revealed that, after controlling for participants' age, gender, and previous counseling experience, only the AAVS-M subscale of emotional self-control continued to contribute unique variance in the prediction of counseling attitudes, $\beta=-.39, p<.001, \Delta R^{2}=.20$. Thus, although all of the AAVS-M value dimensions were significantly associated with participants' total ATSPPH scores, it 
appears that emotional self-control may be the best single predictor of Asian and Asian international students' counseling attitudes, accounting for as much as $20 \%$ of the construct variance. A strength of this study was the use of the psychometrically superior AAVS-M, as opposed to the older, but more commonly used AVS. A limitation of this study, however, was the fact that Asian American and Asian international students were combined in one sample, without considering possible within-group differences.

In conclusion, the studies reviewed above indicate that cultural values may be an important predictor of help-seeking constructs among Asian and Asian American populations. These findings, however, need to be qualified in light of several methodological and conceptual limitations. To begin with, most of the studies listed above used the original AVS scale rather than its psychometrically superior revision, the AAVS-M (Kim et al., 2005). In fact, I was able to locate only one study which used the newer and psychometrically superior AAVS-M (Zayco, 2009). Another limitation is that most of the studies failed to report the participants' citizenship status, leaving it unclear if Asian international students were represented in this research. In fact, only two studies reported data on participants' citizenship status, indicating that a small percentage of the sample consisted of Asian international students (Liao et al., 2005; Zayco, 2009). Finally, the research literature tended to focus primarily on the relationship between Asian values and counseling attitudes, while directing relatively less attention to other relevant helpseeking constructs, such as stigma concerns and intentions to seek counseling.

Given these limitations, future research should examine the relationship between Asian values and psychological help-seeking using the more recently developed and psychometrically superior AAVS-M scale (Kim et al., 2005). In addition, because 
researchers cannot assume that U.S.-born Asian American and foreign-born Asian international students represent the same population, future research should examine Asian international students as a group in its own right. Specifically, future research needs to examine adherence to cultural values as a predictor of different help-seeking constructs (e.g., attitudes, stigma concerns, and intentions) using large samples of Asian international students specifically. Finally, future investigations need to examine Asian values in combination with other cultural factors that may be relevant to an Asian international student population. One such potentially relevant cultural variable is loss of face, an important interpersonal consideration in many Asian societies.

\section{Loss of Face Concerns and Seeking Counseling}

Over the last several decades, multicultural scholars have started to pay increasing attention to the Asian concept of "face." $\mathrm{Hu}$ (1944) was one of the first to define this construct in Asian culture, suggesting that face represents the person's social position or prestige gained by performing one or more specific social roles. Similarly, Goffman (1955) defined face "as an image of self delineated in terms of approved social attributes" (p. 213, as cited in Zane \& Yeh, 2002). More recently, Ho (1991) discussed face as an individual's "moral and social character that constitutes his or her identity, generating a personal sense of dignity, integrity, and self-respect" (p. 120). Furthermore, Ho suggested that social situations in Asian cultures frequently demand that individuals preserve their own and other people's faces in order to promote group harmony and cohesion. Loss of face may occur if an individual's social or moral character is put in question, leading to potential loss of that person's status in the community. In fact, loss of face in Asian culture typically creates feelings of shame, because losing face strips the individual from 
his or her self-respect and social integrity (Ho, 1991). Furthermore, Zane and Yeh (2002) have suggested that loss of face concerns may overlap partially with public shame, such that losing face may bring about shame and dishonor to oneself and one's family.

Consistent with this conceptualization, Ho (1991) suggested that, in Asian culture, loss of face has negative implications not only for the individual, but also for his or her family and community. Given the collectivistic, interdependent nature of many Asian societies, loss of face may bring about shame and dishonor not only to oneself, but also to one's family members and the larger community. In fact, Zane and Yeh (2002) wrote that "face has esteem implications that extend beyond the individual to that of the individual's reference group" and suggested that this is "probably the main reason it has such psychological power in certain shame-based societies such as East Asian cultures" (p. 126). Furthermore, Zane and Mak (2003) suggested that seeking psychotherapy may be construed as face-threatening behavior in many Asian cultures and might be especially salient for less acculturated Asian or Asian American clients. For example, Zane and Yeh (2002) stated that "face loss concerns and shame issues may be especially salient for Asian American clients seeking help for mental health problems that tend to be highly stigmatized issues in their communities" (p. 126). Thus, loss of face concerns may be particularly relevant in understanding Asian international students' counseling use.

Given the cultural relevance of this construct for many Asian and Asian American clients, Zane and Yeh (2002) developed the Loss of Face Scale (LOF), a 21-item unidimensional measure of loss of face concerns. The LOF assesses concerns with losing face in relation to one's social status, ethical behavior, social propriety, or self-discipline in the context of traditional Asian culture. To my knowledge, this is the only measure of 
loss of face concerns in the current psychological literature. In support of the measure's construct validity, Zane and Yeh found that LOF scores were significantly correlated in the expected directions with other conceptually related variables, such as otherdirectedness, private and public self-consciousness, social anxiety, and social desirability. In addition, Asian American college students $(M=91.8, S D=16.9 ; n=81)$ scored significantly higher than did European American students $(M=80.4 ; S D=16.3 n=77)$ on the LOF scale, $t=4.32, p<.001, d=.64$, indicating further support for the scale's validity. In fact, between-group differences on the LOF persisted even after controlling for ethnic differences in other-directedness, private and public self-consciousness, social anxiety, and social desirability, $F(1,153)=12.07, p<.01$. Finally, the LOF scale demonstrated good internal consistency $(\alpha=.83)$ in a sample of 81 Asian American college students, indicating that the scale may be appropriate for use in future research.

Further research has suggested that the newly developed LOF scale relates in the predicted direction to other cultural factors relevant to Asian and Asian American populations. For example, Zane and Yeh (2002) found a significant association between LOF and SL-ASIA scores in a sample of 81 Asian American college students $(r=-.37, \mathrm{p}$ $<.001$ ), indicating that more highly acculturated Asian American students report fewer loss of face concerns. Similarly, Kim et al. (2005) found that LOF scores correlated in the expected direction with scores on the newly developed AAVS-M as well as with each of the AAVS-M subscales in a sample of 189 Asian American college students. The LOF scale was significantly correlated with total AAVS-M scores $(r=.37, p<.001)$, as well as with each of the AAVS-M subscales: collectivism $(r=.17, p<.05)$, conformity to social norms $(r=.20, p<.01)$, emotional self-control $(r=.37, p<.001)$, family 
recognition through achievement $(r=.25, p<.001)$, and humility $(r=.17, p<.05)$.

Specifically, Asian American students who endorsed higher levels of the above cultural values also were likely to report higher levels of loss of face concerns. These findings suggest that the newly developed LOF scale assesses an important construct for many Asians and Asian Americans, and, thus, may contribute to future research.

Given the availability of the newly developed LOF scale, it is important to examine this construct in relation to Asian international students' counseling attitudes, stigma concerns, and help-seeking intentions. Unfortunately, the existing literature on loss of face concerns and help-seeking is scant and has focused primarily on Asian American populations (Zane \& Mak, 2003). In fact, I was able to locate only three studies that surveyed Asian American students (i.e., Chou, 2000; Gong, Gage, \& Tacata, 2003; Leong, Wagner, \& Kim, 1995), and only one study that used a combined Asian American and Asian international sample (i.e., Zayco, 2009). Furthermore, I was unable to locate any studies which explored loss of face concerns with samples of Asian international students specifically. Given this scarcity of research, I will review the currently available studies, while recognizing that the reported findings may not necessarily generalize to Asian international students. I will then use this discussion to argue for exploring loss of face as a help-seeking predictor in future research with Asian international students.

One of the first studies exploring loss of face concerns in the context of helpseeking was conducted by Leong and colleagues (1995) who used the LOF scale as a predictor of Asian American students' expectations about group counseling. The participants were 134 Asian American college students of the following ethnicities: Chinese (33\%), Southeast Asian (27\%), Korean (15\%), Japanese (12\%), and other (13\%). 
The authors failed to report data on respondents' citizenship status, leaving it unclear if any Asian international students were included. Participants completed a demographic questionnaire, the Group Therapy Survey (assessing group counseling expectations), and the LOF scale. Contrary to the authors' hypotheses, LOF scores were not significantly related to Asian American students' expectations about group counseling $(r=-.11, p$ $>.05)$, although the relationship was in the expected inverse direction. One limitation of this study was that the Group Therapy Questionnaire may have assessed participants' expectations, rather than attitudes, toward group counseling. Thus, it is unclear whether these null findings are due to an inadequate construct operationalization or to the absence of a true relationship between loss of face concerns and group counseling attitudes. In addition, the authors failed to report the citizenship status of the study participants, leaving it unclear if any Asian international students were represented in the sample. In a more recent study, Chou (2000) examined loss of face as a predictor of treatment acceptability, willingness to see a counselor, and counselor preferences in a sample of 215 Asian American college students. Participants represented the following ethnicities: Chinese (25\%), Korean (19\%), Asian Indian (15\%), Vietnamese (11\%), Filipino (11\%), Japanese (4\%), and other (15\%). All participants identified as U.S. citizens or permanent residents, with Asian international students being excluded from the sample. Participants completed a demographic questionnaire, the LOF scale, the Treatment Evaluation Inventory, the Willingness to Seek Counseling Questionnaire, and the Counselor Preference Scale. Hierarchical multiple regression indicated that, after controlling for demographic factors (i.e., age, gender, generation status in the U.S., and prior therapy experience), LOF scores failed to emerge as a significant unique predictor 
of treatment acceptability $\left(\beta=.10, p>.05, R^{2}=.01\right)$, or of willingness to seek counseling $\left(\beta=.00, p>.05, R^{2}=.00\right)$. However, LOF scores emerged as a significant unique predictor of participants' counselor preferences, such that students who endorsed greater loss of face concerns expressed stronger preferences for an ethnically similar counselor ( $\beta$ $\left.=.18, p<.05, R^{2}=.03\right)$, as well as for a counselor who holds similar cultural values $(\beta=$ $\left..15, p<.05, R^{2}=.02\right)$. These findings, however, may not generalize to Asian international students, as these students were excluded from the participant sample.

In another recent study, Gong and colleagues (2003) examined loss of face as a predictor of recent help-seeking behavior in a community sample of 2,230 Filipino Americans living in San Francisco, California (42\%) and in Honolulu, Hawaii (58\%). Participants completed a demographic questionnaire, the LOF scale, and the Symptom Checklist-90-Revised. Participants also reported their frequency of utilizing the following resources for help with psychological problems: family and friends, mental health workers, medical professionals, and folk healers. Logistic regression indicated that, after controlling for demographic factors (i.e., age, gender, education, and English fluency) and psychological distress, LOF scores significantly predicted help-seeking from mental health workers $(O R=-.33, p<.01)$, but not from friends and family $(O R=.09, p>.05)$, medical professionals $(O R=-.00, p>.05)$, or folk healers $(O R=-.04, p>.05)$. Thus, it appears that in this Filipino American community sample, loss of face concerns predicted lower probability of consulting a mental health professional, but were unrelated to the probability of seeking help from other sources. A possible explanation of these findings is that, among Filipino Americans, seeking professional mental health services may be more heavily stigmatized than seeking help from other sources, giving rise to greater 
concerns with losing face. This interpretation is speculative, however, given that the authors failed to assess participants' counseling stigma fears. In addition, it is unclear if these findings would generalize to a college sample of Asian international students.

Whereas the previous three studies utilized exclusively Asian American samples, Zayco (2009) examined loss of face concerns and counseling attitudes in a combined sample of 199 Asian American and Asian international students. Participants' demographic characteristics were reported earlier in this chapter and, thus, will not be repeated here. The majority of the participants (94\%) self-identified as U.S. citizens or permanent residents, with a small minority (6\%) identifying as Asian international students. All participants completed a demographic questionnaire, the AAVS-M, the LOF, and the original 29-item ATSPPH scale. Bivariate correlations indicated that participants' LOF scores were significantly associated with total ATSPPH scores $(r=$ $.22, p<.01)$, as well as with two of the ATSPPH subscales: stigma tolerance $(r=-.29, p$ $<.01)$ and interpersonal openness $(r=-.36, p<.01)$. However, when LOF and AAVS-M subscale scores were entered together in a hierarchical multiple regression, only the AAVS-M subscale of emotional self-control emerged as a significant unique predictor of total ATSPPH scores, $\beta=-.39, p<.01, R^{2}=.20$, with the effect of LOF becoming nonsignificant, $\beta=.07, \mathrm{p}>.05, R^{2}=.00$. These results indicate that emotional self-control may mediate the relationship between loss of face and counseling attitudes, such that Asian American and Asian international students who experience stronger loss of face concerns place greater value on emotional self-control, leading to more negative counseling attitudes. A limitation of this study was combining Asian American and Asian international students in the same sample, while failing to examine potential within-group 
differences Furthermore, because Asian international students represented only a small percentage of the entire sample, the findings may not generalize fully to this population.

The research literature reviewed above presents a complex and somewhat contradictory pattern of empirical findings. Furthermore, the reviewed studies suffer from a number of conceptual and methodological limitations. Specifically, none of these investigations included a substantial number of Asian international students, leaving it unclear whether findings generalize to this unique population. Furthermore, although each study explored different help-seeking constructs (e.g., counseling attitudes, stigma tolerance, treatment acceptability, willingness to seek counseling, and past help-seeking behavior), there was considerable variability in the way in which these constructs were measured, possibly leading to contradictory and inconsistent findings. In addition, with a single exception (Zayco, 2009), none of these studies examined loss of face concerns in combination with other cultural variables, such as Asian values or acculturation. Finally, none of these studies used a comprehensive theoretical model that could integrate a wide range of cultural and cognitive predictors of help-seeking.

Given these limitations, future research should explore loss of face as a predictor of Asian international students' counseling attitudes, stigma concerns, and help-seeking intentions. Such research should employ well-validated and psychometrically-sound measures of the above theoretical constructs, such as the abbreviated ATSPPH-SF (Fisher \& Turner, 1995), the Perceptions of Stigmatization by Others Scale (PSOSH; Vogel et al., 2009), and the Intentions to Seek Counseling Inventory (ISCI; Cash, Bagley, McCown, \& Weise, 1978). In addition, loss of face concerns need to be studied in combination with other cultural factors, such as acculturation and Asian values. Such 
research would help elucidate the unique effects of different cultural predictors of helpseeking among Asian international students. Finally, future research should integrate these cognitive and cultural predictors in a theoretically grounded conceptual model. One theoretical framework that could provide such conceptual grounding in future research is the Theory of Reasoned Action (Ajzen \& Fishbein, 1980; Fishbein \& Ajzen, 2009).

\section{The Theory of Reasoned Action as a Framework}

The Theory of Reasoned Action (TRA, Ajzen \& Fishbein, 1980; Fishbein \& Ajzen, 2009) was developed in the 1980s with the aim of predicting and understanding a wide range of behaviors which are under individuals' volitional control. The authors of TRA assumed that people generally act in thoughtful, systematic, and rational ways, by considering their attitudes, social norms, and intentions before engaging in action. Given the assumption of a "reasoned" action approach, Ajzen and Fishbein (1980) and Fishbein and Ajzen (2009) argued that it is possible to predict and understand a person's voluntary behavior based on a limited number of cognitive predictors. These authors further went on to list three cognitive precursors of volitional behavior: 1) Behavioral intentions, or one's stated desire to perform a behavior; 2) Personal attitudes about the costs and benefits of performing the behavior; and 3) Subjective norms about the social appropriateness of the behavior (see Figure 2.1). 
Figure 2.1 Theory of Reasoned Action Model

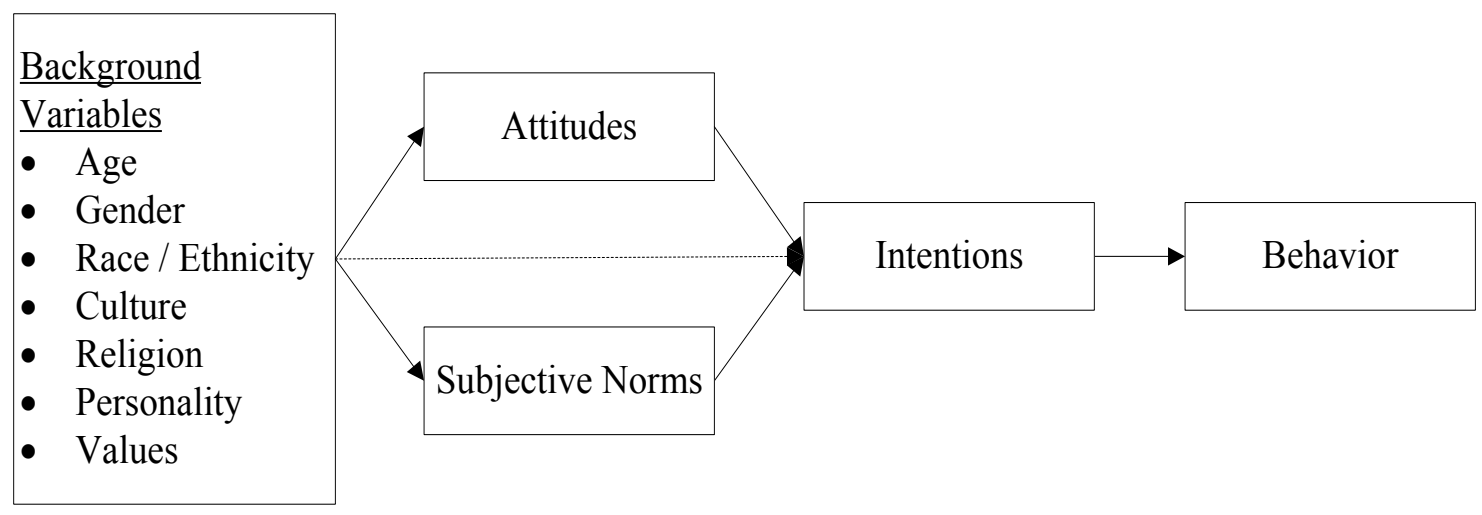

According to the TRA model presented above, human behavior is viewed as a function of individuals' behavioral intentions. Because people are seen as rational and thoughtful actors, the TRA suggests that "a person's intention to perform (or not perform) a behavior is the immediate determinant of the action" (Ajzen \& Fishbein, 1980, p. 5). Under the TRA, intentions are defined as immediate precursors of actions or as "the motivational factors that influence a behavior" (Ajzen, 1991, p. 181). Although Ajzen and Fishbein (1980) acknowledged that intentions don't always translate into corresponding actions, they argued that "barring unforeseen events, a person will usually act in accordance with his or her intention," suggesting that intentions may be the best available predictor of future behavior (p. 5). Thus, according to the TRA, it appears that an individual's intentions to seek counseling for a psychological problem may be the best available predictor of actual mental health service utilization. Under this assumption, it may be possible to predict future counseling utilization rates simply by assessing people's current help-seeking intentions (Vogel et al., 2005).

The TRA model presented above goes on to suggest that behavioral intentions are, in turn, influenced by people's attitudes toward performing the behavior in question. 
Ajzen and Fishbein (1980) defined attitudes as "the individual's positive or negative evaluation of performing the behavior" or as "the person's judgment that performing a behavior is good or bad" (p. 6). According to the TRA, attitudes influence future behavior indirectly through their impact on behavioral intentions (Fishbein \& Ajzen, 2009). In other words, individuals who hold more positive attitudes toward performing a behavior are likely to develop stronger behavioral intentions, thus leading to eventual action. If the above premise is applied to help-seeking, it would follow that counseling attitudes should predict mental health service utilization indirectly through their effect on help-seeking intentions. Thus, a person who experiences psychological difficulties and holds more positive attitudes toward seeking counseling would be more likely to develop strong help-seeking intentions, eventually leading to counseling service utilization.

Besides being influenced by individual attitudes, behavioral intentions also are determined by people's subjective social norms. Ajzen and Fishbein (1980) defined subjective norms as the "person's perception of the social pressures put on him or her to perform or not perform the behavior in question" (p. 6). These authors also argued that an individual's cognitive appraisal is a stronger behavioral predictor than social norms per se, emphasizing the view that cognition is the primary precursor of behavior. By applying this TRA premise to help-seeking, it follows that people's intentions to seek counseling would be influenced by their perception of how significant others would evaluate this behavior. For example, if an individual believes that important others would approve of the decision to seek counseling, he or she would develop stronger intentions to do so. If, on the other hand, the individual believes that seeking counseling carries social stigma, he or she would report lower intentions to seek professional mental health help. Thus, 
under the TRA, it appears that stigma concerns may represent a form of subjective social norm, such that seeking counseling is seen as socially inacceptable.

Finally, Fishbein and Ajzen (2009) suggested that, although people's background characteristics (e.g., age, gender, race/ethnicity, culture, etc.) may impact future behavior, they usually do so indirectly through their effects on attitudes, subjective norms, and behavioral intentions. Fishbein and Ajzen chose not to provide a comprehensive list of cultural and demographic factors that could be relevant in a TRA model, stating that these would vary based on the specific behavioral domain under investigation. Instead, they allowed the individual researcher to determine which background characteristics could be most pertinent in the prediction of a particular behavior of interest. Given my interest in Asian international students' intentions to seek counseling, it would appear that several cultural factors (e.g., acculturation, Asian values, and loss of face), could be included in a culturally-relevant TRA model. Consistent with the basic premises of the TRA, these cultural variables would predict help-seeking intentions indirectly through their effect on personal attitudes, subjective social norms, and behavioral intentions.

Given the overview of the TRA presented above, it appears that this theoretical model may be easily applicable to the counseling help-seeking literature. In fact, several authors have already used the TRA as a framework for understanding individuals' counseling attitudes, stigma concerns, and help-seeking intentions (Bayer \& Peay, 1997; Halgin, Weaver, \& Donaldson, 1985; Halgin, Weaver, Edell, \& Spencer, 1987; Vogel et al., 2005). Unfortunately, most of these studies utilized predominantly European American college student samples. In fact, I was able to locate only three investigations that tested the TRA with samples of Asian and Asian American participants (Christopher, 
Skillman, Kirkhart, \& D’Souza, 2006; Mo \& Mak, 2009; Yong, 2007). Furthermore, I was unable to find any studies examining the TRA as a help-seeking framework with an Asian international college sample. Given these limitations, I will review the available literature on the TRA and help-seeking, while recognizing that the reported results may not generalize to the population of Asian international students in the U.S.

Halgin and colleagues (1985) conducted one of the earliest studies of the TRA in a help-seeking context by examining college students' $(N=147)$ perceptions of the costs, benefits, and social norms surrounding counseling. Participants read a vignette of common depressive symptoms and then listed the pros and cons of obtaining therapy if they, themselves, experienced those symptoms. Participants also listed significant others who would disapprove of their decision to seek counseling. The following perceived benefits of therapy were listed: achieve problem resolution (64\%), understand the causes of depression (59\%), learn how to cope (33\%), and prevent further distress (32\%). In contrast, the following costs of therapy emerged: spend time and money (41\%), experience social stigma (38\%), and confront painful issues (38\%). Participants also listed the following significant others who would disapprove of one's decision to seek counseling: acquaintances $(27 \%)$, friends $(18 \%)$, family $(16 \%)$, parents $(12 \%)$, siblings (6\%), romantic partner (6\%), co-workers (5\%), and employer (3\%). A limitation of the study is the use of a mostly European American college sample, which makes it difficult to generalize the results to a minority population, such as Asian international students.

Subsequently, Halgin et al. (1987) used the TRA to examine the relationships among depression, prior therapy experiences, counseling attitudes, and help-seeking intentions in a predominantly European American college sample $(N=126)$. Participants 
completed a demographic questionnaire, the Beck Depression Inventory (BDI), a single item assessing intentions to seek counseling, and a 6-item measure of counseling attitudes created for the purpose of the study. Based on their help-seeking history and BDI scores, participants were classified into three groups: non-depressed non-seekers $(n=68)$, depressed non-seekers $(n=39)$, and depressed seekers $(n=19)$. MANOVA analysis showed significant group differences in participants' counseling attitudes, $F(2,105)=$ $4.76, p<.01$, and help-seeking intentions, $F(2,119)=21.43, p<.001$. Specifically, nondepressed non-seekers had the least positive attitudes and the lowest help-seeking intentions ( $M=0.83$ and $M=-1.82$, respectively), followed by the depressed non-seekers $(M=0.97$ and $M=0.80)$, and the depressed seekers $(M=1.64$ and $M=1.69) . \mathrm{A}$ limitation of this study is the use of two newly designed, unvalidated help-seeking measures, together with the use of a predominantly European American sample. In another study, Bayer and Peay (1997) used the TRA to predict community outpatients' $(N=142)$ intentions to seek professional mental health services. Participants completed a demographic questionnaire, a single item assessing intentions to seek counseling, a single item assessing subjective norms regarding the acceptability of counseling, and a three-item scale of counseling attitudes. All of these measures listed were created for the purpose of the study. Multiple regression analysis indicated that respondents' intentions to seek counseling were significantly predicted by their helpseeking attitudes $(\beta=.57, p<.001)$, and subjective norms $(\beta=.34, p<.001)$. Taken together, help-seeking attitudes and subjective norms accounted for $34 \%$ of the variance in intentions, $R^{2}=.59, F(2,127)=33.13, p<.001$. Help-seeking attitudes were the more important predictor of intentions, uniquely contributing $23 \%(t=6.69, p<.001)$ of the 
$34 \%$ accounted for by the two independent variables, while subjective norms offered a unique contribution of only $3 \%(t=2.25, p<.05)$. The two independent variables shared $8 \%$ of the explained variance in intentions. These findings indicate that community outpatients who hold positive counseling attitudes and perceive mental health helpseeking as socially acceptable may have stronger intentions to seek counseling in the future. The findings of this study are limited, however, by the use of unvalidated measures and the selection of a predominantly European American sample.

More recently, Vogel and colleagues (2005) used the TRA to examine European American college students' $(N=354)$ counseling attitudes and intentions. Participants completed the ATSPPH scale, the Intentions to Seek Counseling Inventory (ISCI), and nine additional measures assessing the following constructs: stigma concerns, treatment fears, discomfort with self-disclosure, self-concealment, anticipated risks, anticipated benefits, social norms, social support, and prior therapy experience. Consistent with the TRA, structural equation modeling analyses indicated that attitudes partially mediated the relationships between six of the above predictors and participants' help-seeking intentions, $\chi^{2}=(493, N=354)=884.52, p<.001, \mathrm{CFI}=.96, \mathrm{SRMR}=.05, \mathrm{RMSEA}=$ .05 . Bootstrapping analyses further confirmed attitudes as a mediator between helpseeking intentions and the following six predictors: stigma concerns, discomfort with self-disclosure, anticipated therapy benefits, social norms, social support, and prior therapy experience. Although these findings are consistent with TRA, it is notable that the authors conceptualized social norms and stigma concerns as background variables, rather than as aspects of subjective norms, as would be the case in a true TRA model. Another limitation is the use of a predominantly European American college sample. 
Although the studies discussed above relied primarily on European American participants, several researchers also have used the TRA as a help-seeking framework for Asian and Asian American populations. For example, Christopher and colleagues (2006) used the TRA as a model for increasing help-seeking among Thai $(n=109)$ and American $(n=85)$ undergraduates. Participants were randomly assigned to one of three groups - normative, behavioral, or no persuasion—-based on the type of persuasive message they were exposed to. Participants in the normative persuasion group read a paragraph that described help-seeking as socially sanctioned by significant others (e.g., teachers and parents). Participants in the behavioral persuasion group read a paragraph describing the personal advantages of help-seeking. A third control group of participants received no persuasive message. Subsequently, participants completed measures of helpseeking attitudes and intentions designed for the present study. ANOVA revealed that Thai students exposed to normative persuasion had significantly more positive helpseeking attitudes and intentions than did Thai students exposed to either behavioral or no persuasion, $F(2,107)=3.49, p=.03, \eta^{2}=.06$. Conversely, American students exposed to behavioral persuasion reported significantly more positive help-seeking attitudes and intentions than did American students exposed to either normative or no persuasion, $F(2$, $82)=4.36, p=.02, \eta^{2}=.10$. The findings of this study suggest that different types of persuasive messages (normative vs. behavioral) may have a differential impact on the students coming from Asian vs. American cultures. A limitation of the study was the use of unvalidated, newly constructed measures of help-seeking attitudes and intentions.

In another recent study, Mo and Mak (2009) used the TRA to predict mental health help-seeking in a Chinese community sample recruited in Hong Kong $(N=941)$. 
Participants completed a demographic questionnaire, a 5-item scale of counseling attitudes, a 3-item scale of subjective norms, and a 3-item scale of help-seeking intentions, all of which were designed for the purpose of the study. SEM analyses suggested that a TRA-based model fit participants' data well, $\chi^{2}(71)=386.90, p<.001$, $\mathrm{CFI}=.95, \mathrm{RMSEA}=.06$, and explained $57 \%$ of the variance in help-seeking intentions. Consistent with the TRA, attitudes emerged as a significant unique predictor of helpseeking intentions $(\beta=.55, p<.001)$, as did subjective norms $(\beta=.26, p<.001)$. Furthermore, subjective norms had both a direct and an indirect effect on help-seeking intentions through their influence on counseling attitudes. Specifically, Hong Kong Chinese who believed that seeking counseling was socially deviant had less positive attitudes, which then translated into lower help-seeking intentions. Some of the limitations of this study included the use of unvalidated measures, as well as the authors' failure to integrate relevant cultural variables. Finally, it is unclear whether the results of this study would generalize to a population of Asian international students.

Finally, Yong (2007) used the TRA as a conceptual framework in examining Chinese American parents' $(N=185)$ intentions to seek counseling for their children. Participants completed a demographic questionnaire, the SL-ASIA, and three 1-item scales measuring counseling attitudes, subjective norms, and help-seeking intentions. All of the above assessments, with the exception of the SL-ASIA, were designed for the purpose of the study. Bivariate correlations indicated that, consistent with the TRA, counseling attitudes were significantly and positively associated with help-seeking intentions $(r=.44, p<.001)$, as were subjective norms $(r=.50, p<.001)$. In addition, counseling attitudes and subjective norms were significantly and positively correlated ( $r$ 
$=.62, p<.001)$, indicating that participants who saw help-seeking behavior as socially acceptable also held more positive attitudes. Multiple regression indicated that acculturation $(\beta=.19, p<.001)$, attitudes $(\beta=.41, p<.001)$, and subjective norms $(\beta=$ $.25, p<.001)$ each emerged as significant predictors of help-seeking intentions. Specifically, more highly acculturated Chinese American parents, who saw help-seeking as socially sanctioned and held positive counseling attitudes, were also more likely to seek counseling for their children. A strength of this study was the addition of a culturally-relevant variable (acculturation) in the tested TRA model. These findings, however, are limited by the use of newly-designed, unvalidated measures. It is also unclear whether the results would generalize to an Asian international student population.

Taken together, this body of literature indicates that the TRA may be applicable as a help-seeking theoretical framework. The studies reviewed above, however, suffer from a number of methodological and conceptual limitations. With a single exception (i.e., Vogel et al., 2005), all of the studies reviewed above used newly developed and unvalidated measures of counseling attitudes, subjective norms, and help-seeking intentions. Furthermore, no study to date has conceptualized counseling stigma concerns as a subset of individuals' subjective norms regarding the acceptability of counseling. Existing research on the TRA as a help-seeking framework also has relied primarily on European American college student samples. In fact, I was able to locate only three studies that tested the TRA as a help-seeking model with Asian or Asian American populations (Christopher et al., 2006; Mo \& Mak, 2009; Yong, 2007). Furthermore, I was unable to identify any studies that tested the propositions of the TRA in the context of Asian international students' intentions to seek counseling. Related to the previous 
limitation is the fact that few studies have attempted to integrate culturally relevant variables into a TRA help-seeking model.

Given the limitations discussed above, further research should examine TRA as a conceptual framework for understanding Asian international students' intentions to seek counseling. Such research should utilize well-researched and validated measures of counseling attitudes and help-seeking intentions, such as the ATPPH-SF (Fisher \& Turner, 1995) and the ISCI (Cash et al., 1978). Furthermore, future investigations should integrate social stigma as a TRA-based help-seeking construct, such that stigma concerns are conceptualized as an aspect of individuals' subjective norms regarding the acceptability of counseling. Finally, future research should integrate a variety of culturespecific variables, such as acculturation, Asian values, and loss of face. Consistent with the TRA, these cultural predictors can be conceptualized as background characteristics influencing help-seeking intentions indirectly through their effect on counseling attitudes and stigma concerns. Finally, future research should examine counseling attitudes and stigma concerns as either partial or complete mediators between the background cultural factors and Asian international students' intentions to seek counseling.

\section{Summary, Theoretical Models, and Hypotheses}

From the literature review presented above, it appears that future research should examine the relationships among three cognitive predictors of counseling use (i.e., attitudes, stigma concerns, and intentions) among Asian international students. In addition, further research also needs to explore cultural variables (i.e., acculturation, Asian values, and loss of face) as predictors of Asian international students' help-seeking. Finally, research should integrate the above cognitive and cultural constructs using a 
conceptually sound theoretical framework. Given these directions for future research, the purpose of this study was threefold. First, this study explored three cognitive variables (i.e., counseling attitudes, stigma concerns, and help-seeking intentions) relevant to Asian international students' counseling use. Second, this study examined three cultural variables (i.e., acculturation, Asian cultural values, and loss of face) as predictors of Asian international students' help-seeking counseling attitudes, stigma concerns, and intentions. Third, the purpose of this study was to determine whether these cognitive and cultural predictors could be integrated into a conceptual model using the Theory of Reasoned Action (TRA, Ajzen \& Fishbein, 1980; Fishbein \& Ajzen, 2009). Specifically, as recommended by Holmbeck (1997) three alternate TRA models were tested in this study: 1) a non-mediated model; 2) a partially mediated model; and 2) a fully mediated model. According to the non-mediated model, I expected a direct relationship between the cultural predictors and intentions to seek counseling, with no need to include counseling attitudes and stigma concerns as mediators. Conversely, the partially mediated model predicted that cultural variables would have both direct and indirect effects on intentions, through the mediation of counseling attitudes and stigma concerns. Finally, according to the fully mediated model, the three cultural variables were expected to impact intentions only indirectly, through the mediation of counseling attitudes and stigma concerns. All three models were consistent with the TRA as outlined by Fishbein and Ajzen (2009), although these authors expressed a preference for the fully mediated model because of its greater parsimony. Thus, it was hypothesized that the fully mediated model would offer a good fit to the data, while providing a more parsimonious description of construct relationships. Finally, it was hypothesized that bootstrapping 
analyses would support the statistical significance of the hypothesized indirect effects (see Table 2.1).

Figure 2.2 Model Integrating Cultural Variables into the TRA

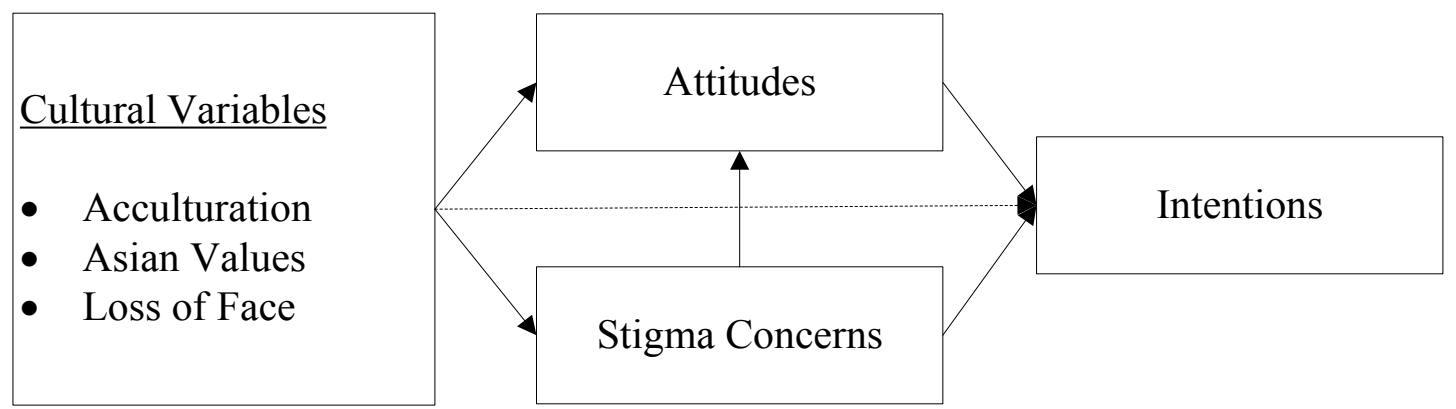


Table 2.1 List of Study Hypotheses

Hypothesis 1: Counseling attitudes and stigma concerns will emerge as significant predictors of help-seeking intentions.

Hypothesis 1a: Counseling attitudes will be positively associated with helpseeking intentions.

Hypothesis 1b: Stigma concerns will be negatively associated with helpseeking intentions.

Hypothesis 1c: Counseling attitudes and stigma concerns will be inversely related to each other.

Hypothesis 2: Cultural variables will emerge as significant predictors of the three helpseeking constructs (i.e., attitudes, stigma concerns, and intentions).

Hypothesis 2a: Acculturation will be negatively associated with stigma concerns and positively associated with attitudes and intentions.

Hypothesis 2b: Asian values will be positively associated with stigma concerns and negatively associated with attitudes and intentions.

Hypothesis 2c: Loss of face will be positively associated with sigma concerns and negatively associated with attitudes and intentions.

Hypothesis 3: The relationships between cultural variables and help-seeking intentions will be mediated by attitudes and stigma concerns.

Hypothesis 3a: A partially mediated TRA model will offer better data fit than a non-mediated model.

Hypothesis 3b: A fully mediated TRA model also will provide a good data fit, while offering greater parsimony than the non-mediated and the partially mediated models.

Hypothesis 3c: Bootstrapping analyses will support the statistical significance of the proposed indirect effects. 


\section{CHAPTER III}

\section{METHOD}

This chapter discusses the methodology used to conduct the present study on Asian international students' intentions to seek counseling. Specifically, this chapter discusses recruitment procedures, questionnaire design, data screening, participant characteristics, and statistical analyses as relevant to the study's implementation.

\section{Recruitment Procedures}

Before initiating participant recruitment, the study was approved by The University of Akron's Institutional Review Board (see Appendix A). Subsequently, participants were recruited using convenience sampling from a list of U.S. colleges and universities with a large international student population. Data from the Open Doors 2009 Report on International Educational Exchange were used to locate the 170 institutions with the largest population of international students (IIE, 2010). Participants were then recruited from the top 75 academic institutions on this list (see Appendix B). Specifically, I obtained the emails of the local international student associations via each school's website and approached the organization officers with a request to forward the attached participant email invitation (Appendix C) to all registered members. In addition, I also contacted each school's Asian ethnic organizations (e.g., Chinese Students and Scholars Association, Japanese Club, Korean Student Organization, Indian Student Organization, etc.) under the assumption that these organizations have a large percentage of Asian 
international students in their membership. This recruitment approach was repeated for each institution until obtaining the desired sample size of at least 180 participants, based on recommendations for conducting path analysis (Kline 2005, see chapter IV). The final sample included 295 Asian international students, which exceeded the minimum recommended sample size for the purpose of the present study.

The email invitation forwarded to potential participants included a description of the study, participant eligibility, the primary investigator's contact information, and an embedded web link to the online survey (see Appendix C). After clicking on the web link, participants were directed to an online questionnaire hosted by SurveyMonkey.com. The first page of the survey consisted of the online informed consent form, which detailed the study's purpose and procedures, eligibility requirements, associated risks and benefits, data anonymity and confidentiality, and the primary investigator's contact information (see Appendix D). Potential participants then read the following question: "Do you agree to participate in this research study?" with two buttons indicating a "Yes" and a "No" option. Respondents who declined participation clicked on the "No" button and exited the survey. Respondents who wished to continue with the survey selected the "Yes" option, documenting their informed consent to participate.

After pressing the "Yes" button, participants were directed to the survey itself and asked to provide their answers to a number of open-ended and Likert-type questionnaires. Upon completion of the survey, participants had the option of providing their first and last names and email addresses to be included in a raffle for one of five \$200 American Express gift cards (see Appendix E). The web page that requested participants' contact information was not associated with the online survey in order to ensure data anonymity. 
Participants also were given the option of withdrawing from the study without penalty at any time simply by closing the online survey web browser.

\section{Measures}

The online survey consisted of a demographics questionnaire and a sequence of measures assessing the following constructs: intentions to seek counseling, attitudes toward seeking counseling, social stigma concerns, acculturation, Asian cultural values, and loss of face concerns. The specific measures and their psychometric properties are outlined below.

Demographics Questionnaire. A demographics questionnaire was included at the beginning of the survey in order to collect data about participants' background characteristic (see Appendix F). It prompted participants to provide information about their age, sex, relationship status, country of origin, ethnicity, and number of years in the U.S. Participants also indicated their U.S. visa type, academic status, and current field of study. In addition, participants responded to three questions assessing their prior history of seeking professional psychological help for a personal-emotional problem. Finally, participants were asked to indicate if they are aware of any on-campus and/or community-based mental health resources for dealing with personal-emotional problems. Participants who responded affirmatively to either of these two questions were prompted to list the mental health resources that they are aware of.

Intentions to Seek Counseling Inventory. The ten-item Psychological and Interpersonal Concerns subscale of the Intentions to Seek Counseling Inventory (ISCI; Cash, Bagley, McCown, \& Weise, 1978) was included in the online survey as a measure of participants' intentions to seek counseling (see Appendix G). The ISCI is a 17-item 
multidimensional questionnaire assessing an individual's intentions to seek counseling for problems that are common in a college population, including relationship difficulties, depression, and personal worries. Items are rated on a Likert-type scale ranging from 1 (very unlikely) to 4 (very likely). Item responses are then summed to produce a total score, with higher scores indicating stronger help-seeking intentions.

Although Cash and colleagues failed to provide reliability and validity data about the ISCI, several researchers have subsequently examined the measure's psychometric properties (Cepeda-Benito \& Short, 1998; Shaffer, Vogel, \& Wei, 2006; Vogel, Wade, \& Hackler, 2007). Cepeda-Benito and Short (1998) conducted a factor analysis on the 17item ISCI using a sample of 732 undergraduates, representing the following ethnic backgrounds: European American (73\%), Hispanic American (14\%), African American (4\%), Asian American (3\%), and Native American (2\%). Based on the data provided by these participants, Cepeda-Benito and Short identified an underlying three-factor structure for the ISCI consisting of: Factor 1 - Psychological and Interpersonal Concerns (10 items, $\alpha=.90)$; Factor 2 - Academic Problems (4 items, $\alpha=.71$ ); and Factor 3 - Drug and Alcohol Problems ( 2 items, $\alpha=.86$ ). The internal consistency of the total ISCI scores in this study was acceptable $(\alpha=.89)$. These authors also found evidence for the ISCI's construct validity, given that total ISCI scores correlated significantly and in the expected direction with participants' general psychological distress level $(r=.23, p<.001)$ and attitudes toward seeking professional psychological help $(r=.35, p<.001)$. Specifically, participants who experienced greater levels of psychological distress and who held more positive counseling attitudes tended to report greater intentions to seek psychological help as expressed in higher total scores on the ISCI (Cepeda-Benito \& Short, 1998). 
Subsequent research also has examined the use of the 10-item ISCI Psychological and Interpersonal Concerns subscale as a stand-alone measure. For example, Shaffer and colleagues (2006) found that the 10-item ISCI - Psychological and Interpersonal Concerns subscale was internally stable $(\alpha=.90)$ in a sample of 821 undergraduate college students. The demographic characteristics of this sample were as follows: European American (91\%), Asian American (2\%), African American (2\%), Latino/a American (2\%), and Multiethnic American (2\%). In this study, the ISCI Psychological and Interpersonal Concerns subscale also correlated significantly and in the predicted direction with participants' counseling attitudes $(r=.54, p<.001)$ and with their perceptions of the risks and benefits associated with counseling $(r=-.10, p<.05$ and $r=.47, p<.001$, respectively), providing evidence for the subscale's construct validity. Specifically, participants who endorsed more positive counseling attitudes and who perceived more benefits and fewer costs to seeking counseling reported greater intentions to seek help for a personal-emotional problem (Shaffer et al., 2006).

Similarly, Vogel and colleagues (2007) presented evidence for the construct validity of the ISCI - Psychological and Interpersonal Problems in a sample of 680 undergraduates. The demographic characteristics of the sample were as follows: European American (90\%), Asian American (4\%), African American (2\%), Hispanic $(2 \%)$, international (1\%), and other (1\%). The 10-item ISCI - Psychological and Interpersonal Problems subscale was internally consistent in this sample $(\alpha=.87)$. In addition, the 10-item subscale correlated significantly and in the expected direction with participants' counseling attitudes $(r=.50, p<.001)$, and self-stigmatization in the context of seeking psychological help $(r=-.37, p<.001)$. In other words, participants who held 
more positive counseling attitudes and expressed lower stigma concerns also endorsed stronger help-seeking intentions for a personal-emotional problem. Given the good psychometric properties of the ISCI - Psychological and Interpersonal Concerns, and the present focus on help-seeking for personal-emotional problems, the ISCI - Psychological and Interpersonal Concerns subscale was administered in the online survey. The Cronbach's alpha coefficient obtained for the ISCI - Psychological and Interpersonal Concerns in the present study was similar to that reported in previous research $(\alpha=.86$; see Table 3.2).

\section{Attitudes Toward Seeking Professional Psychological Help Scale-Short}

Form. The Attitudes Toward Seeking Professional Psychological Help Scale - Short Form (ATSPPH-SF; Fisher \& Farina, 1995) was included in the online survey as a measure of participants' counseling attitudes (see Appendix H). The ATSPPH-SF is a 10item unidimensional measure derived from the original 29-item Attitudes Toward Seeking Professional Psychological Help Scale (Fischer \& Turner, 1970). Responses on the 10-item abbreviated scale are scored on a 4-point Likert-type scale from 1 (disagree) to 4 (agree). Item responses are summed to produce a total ATSPPH-SF score, with higher scores reflecting more positive attitudes toward seeking professional counseling.

Evidence of the ATSPPH-SF's unidimensional structure, internal consistency, test-retest reliability, and construct validity has been presented by Fisher and Farina (1995) and by Elhai, Schweinle, and Anderson (2008). Fischer and Farina (1995), for example, conducted a factor-analysis of the ATSPPH-SF scale using data obtained from 389 undergraduates. These authors failed to provide information about participants' ethnic background, so the ethnic composition of the sample is unknown. Subsequent 
factor analyses yielded a one-factor solution for the 10-item ATSPPH-SF, supporting the hypothesized unidimensional structure of the measure. The ATSPPH-SF also demonstrated adequate internal consistency in this sample $(\alpha=.84)$, lending initial evidence to support the measure's reliability. In addition, the abbreviated ATSPPH-SF scale demonstrated good test-retest reliability over a four-week period $(r=.80)$ in a sample of 62 undergraduate college students (Fischer \& Farina, 1995). Finally, in support of the construct validity of the new measure, Fischer and Farina found that the original ATSPPH and the abbreviated ATSPPH-SF scales were highly correlated $(r=.87)$, suggesting that the two measures assessed the same underlying construct.

More recently, Elhai and colleagues (2008) presented further reliability and validity evidence for the 10-item ATSPPH-SF. Data were obtained from 296 undergraduates representing the following ethnicities: European American (93\%), Asian American (3\%), Native American (3\%), and Hispanic American (1\%). The internal consistency of the ATSPPH-SF scale was acceptable $(\alpha=.77)$. The construct validity of the ATSPPH-SF scale was supported by significant correlations in the expected direction with participants' stigma concerns $(r=-.44, p<.001)$, intentions to seek mental health care in the next month $(r=.24, \mathrm{p}<.001)$, and intentions to seek mental health care in the next 6 months $(r=.26, p<.001)$. In addition, respondents' ATSPPH-SF scores helped differentiate between participants who had used mental health services in the past 6 months $(M=20.50, S D=5.21)$, as compared to those who had not accessed such services $(M=16.63, S D=5.09), F(1,294)=19.00, p<.001$. Specifically, participants who had sought counseling in the past endorsed more positive counseling attitudes, as indicated by higher ATSPPH-SF scores. Given the good psychometric properties of the abbreviated 
ATSPPH-SF, this scale was selected as the most appropriate measure of participants' attitudes toward seeking counseling in this study. The Cronbach's alpha coefficient for the ATSPPH-SF obtained in the present study was somewhat lower than that reported in previous research ( $\alpha=.75$; see Table 3.2), although it was still in the accepted range (Tabachnick \& Fidell, 2007).

\section{Perceptions of Stigmatization by Others for Seeking Psychological Help}

Scale. The Perceptions of Stigmatization by Others for Seeking Psychological Help Scale (PSOSH; Vogel, Wade, \& Ascheman, 2009) was included in the online survey as a measure of participants' social stigma concerns in the context of seeking counseling (see Appendix I). The PSOSH is a 5-item unidimensional scale designed to assess individuals' fears that they will be viewed negatively and/or stigmatized by significant others in their social network in the event of seeking professional psychological counseling (Vogel et al., 2009). Responses on the PSOSH are recorded on a 5-point Likert-type scale ranging from 1 (not at all) to 5 (a great deal). Item responses are summed to produce a total PSOSH score, with higher scores indicating greater overall fear of being stigmatized by significant others in the event of seeking counseling.

Evidence of the scale's undimensional structure, internal consistency, test-retest reliability, and construct validity has been presented by Vogel et al. (2009). Specifically, these authors examined the psychometric properties of the PSOSH using data from three undergraduate college student samples. In Study 1, the authors conducted a factor analysis of the PSOSH using an ethnically diverse sample $(N=842)$ with the following demographic characteristics: European American (51\%), Asian American (14\%), African American (10\%), Latino/a American (8\%), Multiethnic American (8\%), international 
students (8\%), and Native American (1\%). Confirmatory factor analysis supported the hypothesized unidimensional structure of the PSOSH scale. In addition, in this sample, the PSOSH demonstrated the following internal consistency coefficients for the total sample and for each ethnic group: total sample $(\alpha=.89)$, European American $(\alpha=.90)$, Asian American $(\alpha=.88)$, African American $(\alpha=.90)$, Latino/a American $(\alpha=.90)$, Multiethnic American $(\alpha=.89)$, international students $(\alpha=.83)$, and Native American $(\alpha$ $=.89)$. Vogel and colleagues (2009) used these data to conclude that the PSOSH scale has demonstrated good internal consistency in this sample.

Vogel et al. (2009) subsequently presented evidence for the construct validity of the PSOSH using data from a second sample of 506 undergraduates. The demographic characteristics of this sample were as follows: European Americans (92\%), Asian Americans (4\%), Latino/a Americans (1\%), African Americans (1\%), Multiethnic Americans (1\%), and international students (1\%). The construct validity of the PSOSH was supported by significant correlations in the expected direction with participants' attitudes toward seeking counseling $(r=-.66, p<.001)$, as well as with participants' perceptions of the public stigma of counseling $(r=.31, p<.001)$, the public stigma of mental illness $(r=.20, p<.001)$, and the internalized self-stigma of seeking help $(r=.37$, $p<.001)$. In addition, factor analysis indicated that although participants' PSOSH scores were highly correlated with the above three stigma measures (i.e., public stigma, selfstigma, and the stigma of mental illness), the PSOSH constituted a construct in its own right and all of the scale's items loaded on a single factor. Vogel and colleagues (2009) used these findings as further evidence in support of the PSOSH's construct validity. 
Finally, Vogel et al. (2009) examined the temporal stability of the newly developed PSOSH scale using data obtained from a third sample of 144 undergraduates. The demographic characteristics of this sample were as follows: European Americans (88\%), Asian Americans (5\%), African Americans (3\%), Multiethnic Americans (2\%), Latino/a Americans (1\%), and international students (1\%). The test-retest reliability coefficient obtained in this sample over a 3 -week period was acceptable $(r=.77, p<$ .001). In addition, participants' PSOSH scores did not change significantly from Time 1 $(M=11.30, S D=3.66)$ to Time $2(M=11.56, S D=3.78 ; p>.05)$. Given its good psychometric properties and its relative brevity, the PSOSH scale was selected as the most appropriate stigma measure for the purpose of this study. In addition, I considered the PSOSH to be the most appropriate measure of stigma concerns, given its focus on individuals' fears of being stigmatized by significant others in their social support network. This definition of stigma concerns is the closest to the construct of subjective social norms, as defined in the TRA (Fishbein \& Ajzen, 2009). Finally, the PSOSH demonstrated good internal consistency in the present sample of Asian international students (Cronbach's $\alpha=.90$; see Table 3.2), which is consistent with previous research.

Asian American Multidimensional Acculturation Scale. The fifteen-item European American subscale of the Asian American Multidimensional Acculturation Scale (AAMAS-EA; Chung, Kim, \& Abreu, 2004) was included as a measure of behavioral acculturation (See Appendix J). The AAMAS is a 30-item scale, developed as a bidimensional acculturation measure for Asian and Asian American populations. It consists of two orthogonal 15-item subscales: Factor 1, the Asian American Multidimensional Acculturation Scale - Country of Origin (AAMAS-CO) and Factor II, 
the Asian American Multidimensional Scale - European American (AAMAS-EA). The AAMAS-CO was developed to assess association with one's culture of origin (i.e., enculturation), whereas the AAMAS-EA was designed to assesses identification with American culture (i.e., acculturation). Responses on the AAMAS-CO and the AAMASEA are recorded on a 6-point Likert-type scale ranging from 1 (not at all) to 6 (very much). Item responses are then summed to produce a total AAMAS-CO and a total AAMAS-EA score, with higher scores indicating greater levels of enculturation and acculturation, respectively. There is no total AAMAS scale score for this measure because the AAMAS-CO and AAMAS-EA dimensions are considered to be orthogonal.

Evidence of the scale's bidimensional structure, internal consistency, test-retest reliability, and construct validity has been presented by Chung et al. (2004). Specifically, these authors examined the psychometric properties of the AAMAS using data from three Asian American college samples. In Study 1, data were collected from 342 Asian American undergraduates who identified with the following ethnicities: Chinese (28\%), Korean (27\%), Japanese (17\%), Filipino (12\%), Vietnamese (11\%), and other Asian ethnicity (8\%). In this sample, the AAMAS-CO and AAMAS-EA yielded good internal consistency coefficients ( $\alpha=.78$ and $\alpha=.81$, respectively). Furthermore, participants' AAMAS-CO and AAMAS-EA scores were essentially unrelated $(r=-.06, p>.05)$, supporting the bidimensional, orthogonal conceptualization of enculturation and acculturation. Finally, an exploratory factor analysis revealed that each of the AAMAS$\mathrm{CO}$ and the AAMAS-EA items loaded on their respective factor, once again supporting the hypothesized bidimensional structure of the measure (Chung et al., 2004). 
In Study 2, Chung and colleagues (2004) tested the construct validity of the AAMAS-CO and the AAMAS-EA with a sample of 138 Asian American undergraduates who represented the following ethnicities: Chinese (30\%), Korean (23\%), Multiethnic Asian (12\%), Filipino (9\%), Asian Indian (9\%), Japanese (9\%), Taiwanese (4\%), and Vietnamese (3\%). The construct validity of the two AAMAS subscales was demonstrated through significant correlations in the expected direction with other conceptually related constructs, such as acculturation (assessed through the SL-ASIA), Asian values (assessed through the AVS), and generation status. For example, participants' AAMAS-CO scores correlated significantly with acculturation $(r=-.75, p<.001)$, endorsement of Asian values $(r=.37, p<.01)$, and generation status $(r=-.17, p<.04)$. Similarly, participants' AAMAS-EA scores correlated significantly with acculturation $(r=.32, p<.01)$ and endorsement of Asian values $(r=-.25, p<.01)$. Chung and colleagues (2008) used these findings as evidence for the construct validity of the two AAMAS subscales.

Finally, in Study 3, Chung and colleagues (2004) examined the test-retest reliability of the two AAMAS subscales in a sample of 44 Korean American undergraduates. In this sample, the AAMAS-CO and the AAMAS-EA demonstrated acceptable test-retest reliability over a 2-week period ( $r=.89$ and $r=.78$, respectively). Given the present focus on participants' level of behavioral acculturation and the sound psychometric properties of the AAMAS-EA, this scale was selected for inclusion in the survey. Specifically, this study focused on participants' behavioral acculturation to U.S. society. The AAMAS-CO was not included because it measures a conceptually different construct, behavioral enculturation (i.e., identification with one's heritage culture), which was not a focus of the present study. Specifically, it was hypothesized that for Asian 
international students, the AAMAS-EA will be more relevant given the rapid behavioral acculturation that is often observed among first-generation immigrants (Kim et al., 1999). In contrast, behavioral enculturation was considered to remain stable across time for first generation immigrants, as suggested by Kim and colleagues (1999). Furthermore, the AAMAS-EA demonstrated good internal consistency in the present sample of Asian international students (Cronbach's $\alpha=.85$; see Table 3.2), which is consistent with prior research.

Asian American Values Scale - Multidimensional. The Asian American Values Scale - Multidimensional (AAVS-M; Kim, Li, \& Ng, 2005) was utilized to assess participants' adherence to traditional Asian values (see Appendix K). The AAVS-M is a 42-item multidimensional scale developed to assess the following five value dimensions: Factor I - Collectivism ( 7 items); Factor II - Conformity to Social Norms ( 7 items); Factor III - Emotional Self-Control (8 items); Factor IV - Family Recognition Through Achievement (14 items); and Factor V - Humility (6 items). According to a confirmatory factor analysis conducted by Kim et al. (2005), the AAVS-M is best represented through a hierarchical factor structure with five lower order factors representing the AAVS-M subscales and an overall higher level factor representing total AAVS-M scores. Consistent with this conceptualization, responses on the AAVS-M are rated on a 7-point Likert-type scale ranging from 1 (strongly disagree) to 7 (strongly agree). Participants' responses are summed to produce the five AAVS-M subscale scores. The five subscale scores can then be summed to produce a total AAVS-M score, with higher scores indicating stronger endorsement of Asian values. For the purpose of this study, only total AAVS-M scores were used, given the focus on overall endorsement of Asian values. 
Evidence of the scale's multidimensional structure, internal consistency, testretest reliability, and construct validity has been presented by Kim and colleagues (2005). Specifically, Kim et al. (2005) examined the psychometric properties of the AAVS-M using data from three different Asian American college student samples. In Study 1, data were collected from 163 Asian American undergraduates representing the following ethnicities: Korean (22\%), Asian Indian (19\%), Chinese (15\%), Filipino (13\%), Multiethnic Asian (11\%), Taiwanese (6\%), Vietnamese (4\%), Japanese (2\%), and other (8\%). In this sample, the internal consistency coefficient for the total AAVS-M score was high $(\alpha=.89)$. Acceptable internal consistency coefficients were also obtained for each of the five AAVS-M subscales: Collectivism $(\alpha=.80)$, Conformity to Norms $(\alpha=.79)$, Emotional Self-Control $(\alpha=.80)$, Family Recognition Through Achievement $(\alpha=.90)$, and Humility $(\alpha=.81)$. Kim et al. (2005) interpreted these findings as initial evidence for the reliability of the AAVS-M and its five subscales.

In Study 2, Kim and colleagues (2005) presented evidence of the construct validity of the AAVS-M based on a sample of 189 Asian American college students representing the following ethnicities: Chinese (31\%), Korean (19\%), Filipino (13\%), Taiwanese (6\%), Vietnamese (6\%), Asian Indian (5\%), Japanese (5\%), Cambodian (2\%), and other (14\%). In this sample, the construct validity of the total AAVS-M scores was demonstrated by significant correlations in the expected direction with other conceptually related constructs, such as interdependent self-construal $(r=.41, p<.001)$, behavioral acculturation $(r=-.12, p<.05)$, behavioral enculturation $(r=.19, p<.05)$, and loss of face concerns $(r=.37, p<.001)$. In addition, each of the five AAVS-M subscales correlated significantly and in the expected direction with these conceptually related 
constructs. For example, Collectivism and Family Recognition showed significant positive correlations with participants' interdependent self construal $(r=.38, p<.001$ and $r=.36, p<.001$, respectively). Similarly, Conformity, Emotional Self-Control, and Humility correlated significantly and positively with loss of face concerns $(r=.20, p<$ $.05 ; r=.37, p<.001$; and $r=.25, p<.001$, respectively). Kim and colleagues (2005) used these findings to support the construct validity of the AAVS-M and its five subscales.

Finally, in Study 3, Kim and colleagues (2005) examined the test-retest reliability of the AAVS-M with a sample of 38 Asian American college students representing the following ethnicities: Korean (21\%), Chinese (16\%), Filipino (13\%), Vietnamese (13\%), Asian Indian (11\%), Taiwanese (8\%), Cambodian (5\%), Multiethnic Asian (3\%), and other $(10 \%)$. In this sample, the test-retest reliability for total AAVS-M scores was high $(r=.92)$ over a 2 -week period. The 2 -week test-retest reliability coefficients for the five AAVS-M subscales were also acceptable: Collectivism $(r=.73)$, Conformity $(r=.76)$, Emotional Self-Control $(r=.92)$, Family Recognition $(r=.92)$, and Humility $(r=.81)$. For the purpose of this study, only total AAVS-M scores were used, given the present focus on participants' overall endorsement of traditional Asian values. The use of total AAVS-M scores is supported by the strong psychometric properties of the measure, as well as by its hierarchical structure (Kim et al., 2005). Furthermore, the AAVS-M was selected, given the importance of assessing cultural values in addition to behavioral acculturation. For example, Kim and colleagues (1999) suggested that researchers need to routinely assess cultural values in relation to acculturation, given that value-based changes occur more slowly than behavioral acculturation for first generation immigrants. 
Finally, the AAVS-M showed good internal consistency (Cronbach's $\alpha=.89$; see Table 3.2) in the present sample, which is consistent with prior research using this scale.

Loss of Face Scale. The Loss of Face Scale (LOF, Zane \& Yeh, 2002) was included in the online survey in order to assess participants' concerns with losing face in a social and interpersonal context (see Appendix L). The LOF is a 21-item unidimensional questionnaire designed to assess concerns with losing one's social integrity in an Asian and Asian American population. Responses on the LOF are recorded on a 7-point Likert-type scale ranging from 1 (strongly disagree) to 7 (strongly agree). Participants' responses to each item are summed to produce a total LOF score, with higher scores indicating greater concerns with losing face in social interactions.

Evidence of the scale's unidimensional structure, internal consistency, and construct validity has been presented by Zane and Yeh (2002) and by Kim and colleagues (2005). Specifically, Zane and Yeh (2002) examined the psychometric properties of the LOF using data obtained from two samples of 77 European American and 88 Asian American college students. The Asian American sample included the following ethnicities: Chinese (41\%), Korean (22\%), Filipino (12\%), Vietnamese (8\%), and Japanese (7\%). Using data from their European American and Asian American participants, Zane and Yeh (2002) conducted a factor-analysis on the LOF, which produced a one-factor solution, supporting the unidimensional structure of the scale for both ethnic groups. In addition, in the Asian American sample, the LOF demonstrated good internal consistency $(\alpha=.83)$, lending initial support for the scale's reliability. Zane and Yeh (2002) failed to report the internal consistency of the LOF scale in the European 
American sample, although they indicated that the scale's reliability was comparable across ethnic group (i.e., Asian and European American).

Subsequently, Zane and Yeh (2002) sought to demonstrate the construct validity of the LOF through significant correlations in the expected direction with other conceptually related constructs, such as other-directedness, public self-consciousness, social anxiety, and extraversion. In the Asian American sample described above, LOF scores correlated significantly with other-directedness $(r=.44, p<.001)$, public selfconsciousness $(r=.42, p<.001)$, social anxiety $(r=.54, p<.001)$, and extraversion $(r=$ $-.28, p<.01$. In addition, participants in the Asian American sample scored significantly higher on the LOF $(M=.91 .8, S D=16.9)$ than did the participants in the European American sample $(M=80.4, S D=16.3), t(156)=4.32, p<.01$, lending further support for the scale's construct validity (Zane \& Yeh, 2002). Unfortunately, Zane and Yeh failed to collect data about the measure's test-retest reliability, and, as a result, the stability of participants' LOF scores across time is unknown.

Finally, additional evidence for the construct validity of the LOF was presented in Study 2 by Kim and colleagues (2005), as described earlier in this chapter in the section on the psychometric properties of the AAVS-M. Specifically, Kim and colleagues found that LOF scores correlated significantly and in the expected direction with total AAVS-M scores $(r=.37, p<.001)$, as well as with each of the AAVS-M subscales: Collectivism $(r$ $=.17, p<.05)$, Conformity to Norms $(r=.20 p<.05)$, Emotional Self-Control $(r=.37, p$ $<.001)$, Family Recognition Through Achievement $(r=.25, p<.001)$, and Humility ( $r=$ $.17, p<.05)$. In addition, in this study, participants' LOF scores correlated significantly and in the expected direction with independent and interdependent self-construal $(r=-$ 
$.43, p<.001$ and $r=.42, p<.001$, respectively). Finally, the LOF demonstrated a good internal consistency in Kim and colleagues' sample $(\alpha=.86)$. The LOF was selected for this study, given its acceptable psychometric properties and the fact that this is the only currently available measure of loss of face concerns. Finally, the Cronbach's alpha obtained in the present study ( $\alpha=.88$; see Table 3.2) was consistent with prior research.

\section{Data Screening}

A total of 591 participants endorsed the informed consent form and started the online survey. After data collection was completed, the data set was screened for participant eligibility, random responding, incomplete surveys, and univariate and multivariate outliers. Eligibility was determined by reviewing respondents' country of origin, ethnicity, and visa type as reported on the demographic questionnaire. Given the present focus on Asian international students, participants were retained if they endorsed an Asian ethnicity, came from an Asian country of origin, and reported an international student visa status. For the purpose of this study, the term "Asian" was used as defined by the U.S. Census Bureau (2007). Specifically, the U.S. Census defined "Asian" as "a person having origins in any of the original peoples of the Far East, Southeast Asia, or the Indian subcontinent” (p. 7). Similarly, in its 2009 Open Doors Report, the Institute of International Education defined Asian as an individual who comes from one of the following regions: 1) East Asia; 2) South and Central Asia; and 3) Southeast Asia.

Given this definition, individuals from the following geographic regions were eligible to participate: 1) East and Southeast Asia (e.g., China, Hong Kong, Japan, Korea, Taiwan, Cambodia, Indonesia, Malaysia, Philippines, Thailand, Singapore, and Vietnam) and 2) South and Central Asia (e.g., Bangladesh, Bhutan, India, Kazakhstan, Kyrgyzstan, 
Pakistan, Nepal, Sri Lanka, Tajikistan, Turkmenistan, and Uzbekistan; IIE, 2010). Participants from the Middle East were excluded, given their unique geographic and cultural background (IIE, 2010). Furthermore, for the purpose of this study, an international student was defined as a college student who holds a foreign student visa (i.e., F-1, J-1, M-1, or H-1 visa type; Coppi, 2007). F-1 visa holders are defined as international students pursuing educational degrees at U.S. institutions of higher education. J-1 visa holders are international exchange visitors who are currently enrolled at foreign universities, but who are completing study abroad programs at U.S. institutions of higher education. M-1 visa holders are defined as international students pursuing vocational training at U.S. colleges and universities. Finally, H-1 holders are defined as international students enrolled part-time at U.S. colleges and universities, while simultaneously working for a U.S. employer (Coppi, 2007). Students who did not meet the inclusion criteria were screened out of the sample. Specifically, 10 participants $(1.7 \%)$ were excluded, because they did not meet the requirements for an Asian country of origin. In addition, 137 participants (23.2\%) were excluded, because they identified as a U.S. resident or permanent citizen.

Screening for random responding relied on three validation items embedded at the end of the AAMAS-EA, the AAVS-M, and the LOF scales. A sample validation item was: "This is a survey validation item. Please select 1 (strongly disagree) as your response for this question.” This procedure has been used previously in online surveys with international students to ensure that participants read each item carefully and understood item content (Wei, Heppner, Mallen, Ku, Liao, \& Wu, 2007). Based on these authors' recommendations, participants who responded incorrectly to one or more of 
these items were excluded from the sample. A total of 45 participants (7.6\%) were excluded from the sample using these criteria.

Furthermore, data also were screened for missing values, following recommendations made by Tabachnick and Fidell (2007). Specifically, surveys with more than $5 \%$ of missing data, either in a single questionnaire or across the entire survey, were excluded from analysis. This resulted in 103 participants (17.4\%) being excluded from the sample, because of substantial amounts of missing data. For surveys with less than $5 \%$ of missing data, sample mean substitution was employed to replace missing values. Specifically, missing values on any item were replaced with the mean for that specific item across the entire sample. Sample mean substitution is considered appropriate when dealing with small amounts of missing data (i.e., less than 5\%), because it does not introduce significant bias in parameter estimates (Tabachnick \& Fedell, 2007).

Subsequently, a MANOVA was conducted to determine if participants who failed to complete a substantial portion of the survey were statistically different on any of the continuous demographic variables (i.e., age and length of stay in the U.S.) from those who completed the entire survey. The analysis indicated no significant differences between survey completers and non-completers, Wilk's $\lambda(2, N=350)=.99, F=.33, p=$ .72. In addition, a series of chi square goodness of fit tests was used to determine if participants who failed to complete the survey were significantly different on any of the categorical demographic variables (i.e., gender, relationship status, academic rank, field of study, U.S. visa type, prior use of counseling, and awareness of on-campus and offcampus mental health resources) as compared to participants who completed the entire survey. These analyses revealed no significant differences between survey completers 
and non-completers on any of the above-listed demographic variables (see Table 3.1 for the obtained chi square estimates and probability values 
Table 3.1 Chi Square Tests for Survey Completers and Non-Completers

\begin{tabular}{lcccc}
\hline Demographic Variable & $\boldsymbol{N}$ & $\chi^{2}$ & $\boldsymbol{d f}$ & $\boldsymbol{p}$ \\
\hline Gender & 398 & 1.37 & 1 & .24 \\
Relationship Status & 398 & 2.50 & 2 & .29 \\
Academic Rank & 370 & 1.12 & 1 & .74 \\
Field of Study & 375 & 2.53 & 4 & .64 \\
Visa Type & 358 & 8.5 & 6 & .20 \\
Prior Counseling & 359 & .65 & 1 & .42 \\
Awareness of On-Campus Resources & 359 & .76 & 1 & .38 \\
Awareness of Off-Campus Resources & 357 & .05 & 1 & .82 \\
\hline
\end{tabular}

Note. The $N$-size reported for each analysis varies because of missing demographic data. Listwise deletion of missing demographic data was used in the above analyses. 
Finally, data were screened for univariate and multivariate outliers using procedures proposed by Tabachnick and Fidell (2007). Specifically, one univariate outlier was identified and deleted based on a $\mathrm{z}$-score value exceeding $z>3.29, p<.001$. Similarly, screening was done for multivariate outliers by examining Mahalonobis distances exceeding the probability of $p<.001$ (Tabachnick \& Fidell, 2007). No multivariate outliers were detected using these criteria. The data set also was examined for multivariate normality to ensure that it met the normality assumption underlying the maximum likelihood procedure used in the subsequent path analyses. Specifically, Finney and DiStephano (2006) have suggested that assumptions of multivariate normality are violated whenever univariate skew and kurtosis exceed the values of 2.0 and 7.0, respectively. The data met the normality assumption defined under these conditions, given that none of the scales exceeded the skew and kurtosis value of 1.0 (see Table 3.2). 
Table 3.2 Descriptive Statistics and Internal Consistencies

\begin{tabular}{lcccccr}
\hline Scale & Range & $\boldsymbol{M}$ & $\boldsymbol{S D}$ & Skew & Kurtosis & Alpha \\
\hline ISCI & $10-37$ & 21.39 & 5.94 & .01 & -.68 & .86 \\
ATSPPH-SF & $12-40$ & 25.87 & 5.05 & .17 & -.15 & .75 \\
PSOSH & $5-25$ & 11.05 & 4.85 & .60 & -.27 & .90 \\
AAMAS-EA & $28-86$ & 58.28 & 10.18 & .09 & -.01 & .85 \\
AAVS-M & $81-254$ & 174.20 & 28.06 & -.23 & .26 & .89 \\
LOF & $41-143$ & 89.67 & 18.09 & .08 & .16 & .88 \\
\hline
\end{tabular}

Note. $N=295$. ISCI $=$ Intentions to Seek Counseling Inventory; ATTSPH-SF $=$ Attitudes Toward Seeking Professional Psychological Help-Short Form; PSOSH = Perceptions of Stigmatization by Others for Seeking Help Scale; AAMAS-EA = Asian American Multidimensional Acculturation Scale-European American subscale; AAVS-M = Asian American Values Scale-Multidimensional; LOF $=$ Loss of Face Scale. 


\section{Sample Characteristics}

After data screening, the final sample consisted of 295 Asian international

students. The demographic characteristics of the sample are presented in Tables 3.3, 3.4, and 3.5. In terms of age, participants ranged from 18 to 42 years $(M=24.8, S D=4.4)$.

With regard to gender, $49.0 \%(n=144)$ identified as men, whereas $51.0 \%(n=150)$ identified as women. A substantial majority were currently single $(76.1 \%, n=198)$, whereas $18.6 \%(n=55)$ identified as partnered, $12.9 \%(n=38)$ as married, $0.3 \%(n=1)$ as divorced, and $1.0 \%(n=3)$ as "other". In terms of academic rank, the majority of the participants were graduate students $(62.0 \%, n=183)$, whereas $34.3 \%(n=101)$ pursued undergraduate studies, and 3.1\% $(n=11)$ identified as "other". The most common field of study was engineering $(36.3 \%, n=107)$, followed by physical and life sciences $(12.2 \%, n=36)$, business and management $(11.5 \%, n=34)$, and social sciences $(10.5 \%$, $n=31)$. The least common fields of study were agriculture $(1.4 \%, n=4)$, education $(1.4 \%, n=4)$, and fine and applied arts $(1.0 \%, n=3)$. Data about participant age, gender, relationship status, academic rank, and fields of study are presented in Table 3.3.

In terms of ethnicity, $98.6 \%(n=290)$ identified with a mono-racial/mono-ethnic Asian background. The remaining 1.4\% $(n=4)$ identified as bi-racial/multi-racial Asian. The most common countries of origin included India $(22.4 \%, n=66)$, China $(21.4 \%, n=$ 63), Taiwan $(12.9 \%, n=38)$, Malaysia $(9.2 \%, n=27)$, and Singapore $(8.8 \%, n=26)$. The least well represented countries of origin were Bangladesh $(0.7 \%, n=2)$, Bhutan $(0.7 \%$, $n=2)$, Sri Lanka $(0.7 \%, n=2)$, the Philippines $(0.7 \%, n=2)$, and Nepal $(0.3 \%, n=1)$. The majority of the respondents held an F-1 student visa $(92.2 \%, n=271)$, with a small minority holding J-1 $(7.1 \%, n=21)$ and H-1 $(.7 \%, n=2)$ visa types. Participants' length 
of stay in the U.S. ranged from 1 to 9 years $(M=2.9, S D=2.2)$. Data about respondents' ethnicity, country of origin, visa type, and years spent in the U.S. are presented in Table

\section{4 .}

Table 3.3 Participant Age, Gender, Relationship Status, Academic Rank, and Field of Study

\begin{tabular}{|c|c|c|}
\hline Variable & $n$ & Percentage \\
\hline \multicolumn{3}{|l|}{ Age $(N=281)$} \\
\hline Minimum & & 18.0 \\
\hline Maximum & & 42.0 \\
\hline Mean & & 24.8 \\
\hline SD & & 4.4 \\
\hline \multicolumn{3}{|l|}{ Gender $(N=294)$} \\
\hline Man & 144 & $49.0 \%$ \\
\hline Woman & 150 & $51.0 \%$ \\
\hline \multicolumn{3}{|l|}{ Relationship Status $(\mathrm{N}=\mathbf{2 9 5})$} \\
\hline Single & 198 & $67.1 \%$ \\
\hline Partnered & 58 & $18.6 \%$ \\
\hline Married & 38 & $12.9 \%$ \\
\hline Divorced & 1 & $.3 \%$ \\
\hline Widowed & 0 & $0 \%$ \\
\hline Other & 3 & $1.0 \%$ \\
\hline \multicolumn{3}{|l|}{ Academic Rank $(N=295)$} \\
\hline Freshman & 10 & $3.4 \%$ \\
\hline Sophomore & 24 & $8.2 \%$ \\
\hline Junior & 37 & $12.5 \%$ \\
\hline Senior & 30 & $10.2 \%$ \\
\hline Graduate & 183 & $62.0 \%$ \\
\hline Other & 11 & $3.7 \%$ \\
\hline \multicolumn{3}{|l|}{ Field of Study $(N=295)$} \\
\hline Business and Management & 34 & $11.5 \%$ \\
\hline Engineering & 107 & $36.3 \%$ \\
\hline Physical and Life Sciences & 36 & $12.2 \%$ \\
\hline Social Sciences & 31 & $10.5 \%$ \\
\hline Math and Computer Science & 14 & $4.7 \%$ \\
\hline Fine and Applied Arts & 3 & $1.0 \%$ \\
\hline Health Care Professions & 17 & $5.8 \%$ \\
\hline Education & 4 & $1.4 \%$ \\
\hline Humanities & 9 & $3.1 \%$ \\
\hline Agriculture & 4 & $1.4 \%$ \\
\hline Other & 36 & $12.2 \%$ \\
\hline
\end{tabular}


Table 3.4 Participant Ethnicity, Nationality, Visa Type, and Years in the U.S.

\begin{tabular}{|c|c|c|}
\hline Variable & $n$ & Percentage \\
\hline \multicolumn{3}{|l|}{ Ethnicity $(N=294)$} \\
\hline Monoracial Asian & 290 & $98.6 \%$ \\
\hline Biracial/Multiracial Asian & 4 & $1.4 \%$ \\
\hline \multicolumn{3}{|l|}{ Nationality $(N=293)$} \\
\hline Bangladesh & 2 & $.7 \%$ \\
\hline Bhutan & 2 & $.7 \%$ \\
\hline China & 63 & $21.4 \%$ \\
\hline Hong Kong & 10 & $3.4 \%$ \\
\hline India & 66 & $22.4 \%$ \\
\hline Indonesia & 10 & $3.4 \%$ \\
\hline Japan & 6 & $2.0 \%$ \\
\hline Malaysia & 27 & $9.2 \%$ \\
\hline Nepal & 1 & $.3 \%$ \\
\hline Pakistan & 11 & $3.7 \%$ \\
\hline Philippines & 2 & $.7 \%$ \\
\hline Singapore & 26 & $8.8 \%$ \\
\hline South Korea & 14 & $4.7 \%$ \\
\hline Sri Lanka & 2 & $.7 \%$ \\
\hline Taiwan & 38 & $12.9 \%$ \\
\hline Thailand & 10 & $3.4 \%$ \\
\hline Vietnam & 3 & $1.0 \%$ \\
\hline \multicolumn{3}{|l|}{ Visa Type $(N=294)$} \\
\hline $\mathrm{J}-1$ & 21 & $7.1 \%$ \\
\hline F-1 & 271 & $92.2 \%$ \\
\hline $\mathrm{H}-1$ & 2 & $.7 \%$ \\
\hline \multicolumn{3}{|l|}{ Years in the U.S. $(N=292)$} \\
\hline Minimum & & 1 \\
\hline Maximum & & 9 \\
\hline Range & & 2.9 \\
\hline SD & & 2.2 \\
\hline
\end{tabular}

In terms of awareness of mental health resources, sixty percent of the respondents $(n=179)$ were aware of on-campus mental health resources, typically listing the local student health center and the university counseling center. Other less commonly listed mental health resources included the office of international programs, academic advisors, and student organizations. Forty percent of the participants $(n=115)$ were not aware of any mental health resources on campus. Furthermore, only $11.6 \%(n=34)$ of the sample stated that they were aware of off-campus mental health services, whereas $88.4 \%(n=$ 259 ) indicated no knowledge of off-campus resources. The most commonly listed offcampus resources included community mental health centers, hospitals, private practice 
mental health professionals, and 24-hour support hotlines. Data about participants' awareness of mental health services and prior use of counseling and are presented in Table 3.5.

Table 3.5 Awareness of Mental Health Resources and Prior Use of Counseling

\begin{tabular}{lrr}
\hline Variable & $\boldsymbol{n}$ & Percentage \\
\hline Awareness of On-Campus Resources $(\boldsymbol{N}=\mathbf{2 9 4})$ & 179 & $60.9 \%$ \\
Yes & 115 & $39.1 \%$ \\
No & & \\
Awareness of Off-Campus Resources $(\boldsymbol{N}=\mathbf{2 9 3})$ & 34 & $11.6 \%$ \\
Yes & 259 & $88.4 \%$ \\
No & & \\
Seen a Mental Health Professional? $(\boldsymbol{N}=\mathbf{2 9 4})$ & 52 & $17.7 \%$ \\
Yes & 242 & $82.3 \%$ \\
No & & \\
Seen a Professional Where? $(\boldsymbol{N}=\mathbf{2 9 4})$ & 14 & $4.7 \%$ \\
In Home Country & 31 & $10.5 \%$ \\
In the U.S. & 7 & $2.5 \%$ \\
Both in Home Country and in the U.S. & & \\
What Type of Professional? $(\boldsymbol{N}=\mathbf{2 9 4})$ & 30 & $10.2 \%$ \\
School Counselor & 13 & $4.4 \%$ \\
Counselor & 3 & $1.0 \%$ \\
Social Worker & 14 & $4.7 \%$ \\
Psychologist & 13 & $4.4 \%$ \\
Psychiatrist & & \\
\hline
\end{tabular}

Finally, in terms of prior use of mental health services, a substantial majority indicated that they had never seen a mental health professional in the past $(82.3 \%, n=$ 242; See Table 3.5). In contrast, a minority $(17.7 \%, n=52)$ indicated that they had prior experience seeking mental health services. Across the entire sample, $10.5 \%(n=31)$ had seen a mental health professional in the U.S., $4.7 \%(n=14)$ had accessed counseling in their home country, and $2.5 \%(n=7)$ had utilized mental health services both in the U.S. and in their home country. The most common source of mental health services was a school counselor $(10.2 \%, n=30)$, followed by a psychologist $(4.7 \%, n=14)$, a counselor 
$(4.4 \%, n=13)$, and a psychiatrist $(4.4 \%, n=13)$. The least common source of counseling services was a social worker $(1.0 \%, n=3)$.

Given that participants who had prior experience seeing a mental health professionals might be substantially different from respondents without such experience, a MANOVA was conducted to examine potential differences on the six cognitive and cultural variables of interest. This analysis produced a significant statistic, Wilk's $\lambda$ ( $6, N$ $=294)=.89, F=6.10, p<.001$, indicating the presence of statistically significant differences between the two groups of participants. Specifically, statistically significant differences were observed on the following three variables: intentions to seek counseling, counseling attitudes, and Asian values (see Table 3.6). An examination of group means revealed that participants who had seen a mental health professional in the past reported significantly stronger help-seeking intentions, more positive counseling attitudes, and less traditional Asian cultural values, as compared to participants with no prior counseling experience (See Table 3.6). These findings are consistent with existing research which has revealed positive associations among past counseling experience, current counseling attitudes, and future-oriented help-seeking intentions (Elhai, Schweinle, \& Anderson, 2008; Fisher \& Farina, 1995; Vogel, Wade, \& Hackler, 2007). Finally, the MANOVA reported above indicated no statistically significant differences on the remaining three variables of interest: stigma concerns, acculturation, and loss of face fears. In addition, a second MANOVA identified no statistically significant differences in terms of where a mental health professional was seen (i.e., in the U.S., in the home country, and both in the U.S. and in the home country), Wilk's $\lambda(18, N=55)=.67, F=1.09, p=.37$. 
Table 3.6 Differences Between Participants With and Without Experience Seeing a Mental Health Professional

\begin{tabular}{|c|c|c|c|c|c|c|c|}
\hline Variable & $M_{y e s}$ & $M_{n o}$ & $M S$ & $d f$ & $F$ & $p$ & $\eta^{2}$ \\
\hline ISCI & 23.99 & 20.83 & 425.55 & 1 & 12.49 & $<.001$ & .04 \\
\hline ATTSPH-SF & 28.75 & 25.23 & 530.93 & 1 & 22.39 & $<.001$ & .07 \\
\hline PSOSH & 11.23 & 10.99 & 2.34 & 1 & .10 & .75 & .00 \\
\hline AAMAS-EA & 60.04 & 57.86 & 202.71 & 1 & 1.96 & .16 & .01 \\
\hline AAVS-M & 163.72 & 176.74 & 7252.99 & 1 & 9.64 & .002 & .03 \\
\hline LOF & 87.69 & 90.21 & 271.96 & 1 & .83 & .36 & .00 \\
\hline \multicolumn{8}{|c|}{ Note. $N=295 . M_{y e s}=$ Participants with Prior Experience Seeing a Mental Health Professional; $M_{n o}=$} \\
\hline \multicolumn{8}{|c|}{ Participants without Prior Experience Seeing a Mental Health Professional; ISCI = Intentions to Seek } \\
\hline \multicolumn{8}{|c|}{ Counseling Inventory; ATTSPH-SF = Attitudes Toward Seeking Professional Psychological Help-Short } \\
\hline \multicolumn{8}{|c|}{ Form; PSOSH = Perceptions of Stigmatization by Others for Seeking Help Scale; AAMAS-EA = Asian } \\
\hline American M & nensiona & culturatio & cale-Euro & erica & scale; & $\mathrm{S}-\mathrm{M}=$ & \\
\hline
\end{tabular}




\section{Main Statistical Analyses}

After data screening, the sample was examined to determine if it was adequate for the purpose of the statistical analyses, using criteria specified by Kline (2005). Subsequently, bivariate correlations were calculated to examine the hypothesized relationships among the constructs of interest. In addition, path analysis was used to compare the Non-Mediated, Partially Mediated, and Fully Mediated Models, as consistent with the TRA (Ajzen \& Fishbein, 1980; Fishbein \& Ajzen, 2009). Specifically, Holmbeck's (1997) three-step method for testing mediated path models was utilized to determine which of the three competing models offered the best overall data fit. Finally, the significance level of the hypothesized indirect effects was evaluated using the bootstrap procedure proposed by Shrout and Bolger (2002). Detail descriptions of these analyses and the corresponding statistical results are provided in Chapter IV. 


\section{CHAPTER IV}

\section{RESULTS}

This chapter describes the results of the present study, which have been organized in the following sections: determining the adequacy of the sample size, testing of the hypothesized bivariate correlations, testing of the hypothesized TRA models, and testing the significance of the hypothesized indirect effects.

\section{Determining the Adequacy of the Sample Size}

After data screening (see Chapter III), the final sample consisted of 295 Asian international students. The adequacy of the sample was determined using criteria for path analysis outlined by Kline (2005). Specifically, Kline recommended the inclusion of 5 to10 participants for each estimated parameter, with an additional recommendation of recruiting at least 100 participants. In the case of the proposed study, 18 free parameters were estimated, resulting in a recommended sample size of 100 to 180 completed surveys. According to these criteria, the present sample was sufficient for the purpose of the study.

\section{Testing the Hypothesized Bivariate Correlations}

Bivariate correlations were calculated to examine the hypothesized relationships among the constructs of interest (see Table 4.1). Specifically, the bivariate correlations among help-seeking intentions, counseling attitudes, and stigma concerns were examined to test Hypotheses 1a, 1b, and 1c. In addition, the bivariate correlations among 
acculturation, Asian values, loss of face concerns and the three help-seeking constructs were examined to test Hypotheses 2a, 2b, and 2c.

Hypotheses $1 \mathrm{a}$ and $1 \mathrm{~b}$ predicted that counseling attitudes would be positively correlated, whereas stigma concerns would be negatively correlated, with participants' help-seeking intentions. Furthermore, hypothesis 1c predicted that counseling attitudes and stigma concerns would be significantly and negatively associated with each other. Data from the correlational analyses indicated support for hypothesis 1a, such that counseling attitudes positively and significantly related to respondents' help-seeking intentions $(r=.38, p<.01)$. Hypothesis $1 \mathrm{c}$ also was supported, such that counseling attitudes and stigma concerns were significantly and inversely related to each other $(r=-$ $.23, p<.01)$. However, the data failed to support Hypothesis $1 \mathrm{~b}$, such that stigma concerns were not significantly correlated with help-seeking intentions $(r=.04, p>.05)$. Thus, there was only partial support for the relationships predicted under Hypothesis 1.

Hypotheses 2a, 2b, and 2c predicted that participants' acculturation, Asian values, and loss of face concerns would be significantly associated with the three help-seeking constructs (i.e., stigma concerns, counseling attitudes, and help-seeking intentions). Specifically, acculturation was hypothesized to be negatively associated with stigma concerns and positively associated with counseling attitudes and help-seeking intentions (Hypothesis 2a). In contrast, Asian values and loss of face were expected to be positively associated with stigma concerns and negatively associated with counseling attitudes and help-seeking intentions (Hypothesis $2 b$ and $2 c$, respectively).

The correlational data analysis failed to provide support for Hypothesis 2a, such that participants' acculturation was not significantly associated with any of the help- 
seeking constructs: stigma concerns $(r=.02, p>.05)$, counseling attitudes $(r=-.01, p>$ $.05)$, and help-seeking intentions $(r=-.09, p>.05)$. In contrast, Hypothesis $2 \mathrm{~b}$ was partially supported, such that participants' endorsement of Asian values was positively correlated with their stigma concerns $(r=.12, p<.05)$ and negatively correlated with their counseling attitudes $(r=-.27, p<.05)$. However, contrary to the hypotheses, Asian values were not significantly associated with intentions to seek counseling $(r=.06, p>$ $.05)$. In addition, the data failed to support Hypothesis $2 \mathrm{c}$, because loss of face concerns were positively, as opposed to negatively, associated with help-seeking intentions ( $r=$ $.21, p<.01)$. Furthermore, the relationship between loss of face and stigma approached, but failed to reach, statistical significance $(r=.11, p=.07)$. Finally, loss of face concerns were not significantly correlated with participants' counseling attitudes $(r=-.09, p>.05)$. Thus, the correlational data provided only partial support for Hypothesis 2 . 
Table 4.1 Bivariate Correlations Among the Constructs of Interest

\begin{tabular}{|c|c|c|c|c|c|c|}
\hline Variables & 1 & 2 & 3 & 4 & 5 & 6 \\
\hline 1. ISCI & - & & & & & \\
\hline 2. ATSSPH-SF & $.38 * *$ & - & & & & \\
\hline 3. PSOSH & .04 & $-.23 * *$ & - & & & \\
\hline 4. AAMAS-EA & -.09 & -.01 & .02 & - & & \\
\hline 5. AAVS-M & .06 & $-.27 * *$ & $.12 *$ & .04 & - & \\
\hline 6. LOF & $.21 * *$ & -.09 & .11 & $-.12 *$ & $.36^{* *}$ & - \\
\hline \multicolumn{7}{|c|}{ Note. $N=295$. ISCI $=$ Intentions to Seek Counseling Inventory; ATTSPH-SF $=$ Attitudes Toward Seeking } \\
\hline \multicolumn{7}{|c|}{ Professional Psychological Help-Short Form; PSOSH = Perceptions of Stigmatization by Others for Seeking Help } \\
\hline \multicolumn{7}{|c|}{ Scale; AAMAS-EA = Asian American Multidimensional Acculturation Scale-European American Subscale; AAVS- } \\
\hline \multicolumn{7}{|c|}{$\mathrm{M}=$ Asian American Values Scale-Multidimensional; LOF = Loss of Face Scale. } \\
\hline 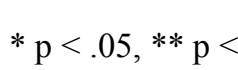 & & 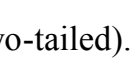 & & & & \\
\hline
\end{tabular}




\section{Testing the Hypothesized TRA Models}

Path analysis was used to test the hypothesized Non-Mediated, Partially

Mediated, and Fully Mediated Models, as outlined in Hypotheses 3a and 3b. Specifically, Hypothesis 3a stated that a Partially Mediated Model would provide a substantially better data fit than a Non-Mediated Model. Furthermore, Hypothesis $3 b$ stated that a Fully Mediated Model also would provide a good data fit, while offering greater parsimony than the Non-Mediated and Partially Mediated Models, thereby making it the best-fitting of the three models examined. To test these hypotheses, path analyses were conducted using Holmbeck's (1997) three-step procedure for testing partial versus full mediation.

The first step of Holmbeck's procedure was to test the significance of the NonMediated Model, which specified that the three cultural variables would directly predict participants' intentions to seek counseling. In this model, the indirect paths through the hypothesized mediators (counseling attitudes and stigma concerns) were set to zero, thus allowing only the direct paths to be freely estimated (see Figure 4.1). The second step involved testing a Partially Mediated Model, which included counseling attitudes and stigma concerns as partial mediators. In this model, no paths were constrained to zero, thus allowing both the direct and indirect paths to be freely estimated (see Figure 4.2). The third step involved testing a Fully Mediated Model, which conceptualized counseling attitudes and stigma concerns as full mediators. In this model, the direct paths were set to zero, thus allowing only the indirect effects to be freely estimated (See Figure 4.3). This approach provided three nested TRA models, which could then be compared statistically to determined overall data fit. The path coefficients obtained after estimating the three competing TRA models are presented in Figures 4.1, 4.2, and 4.2. 
Figure 4.1 Non-Mediated TRA Model

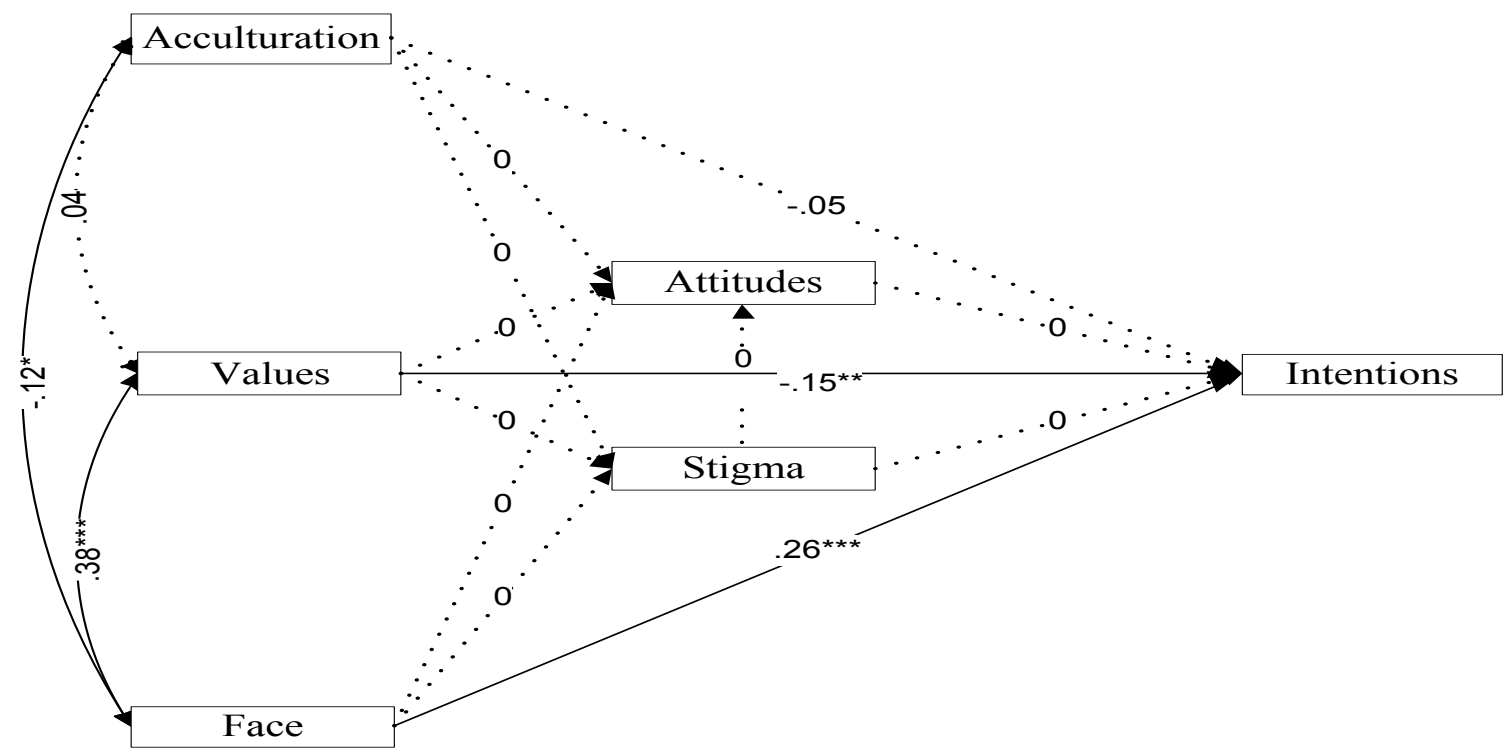

Note. $N=295$. Solid arrows represent statistically significant effects. Dashed arrows represent non-significant effects.

$*<.05 ; * *<.01 ; * * *<.001$ 
Figure 4.2 Partially Mediated TRA Model

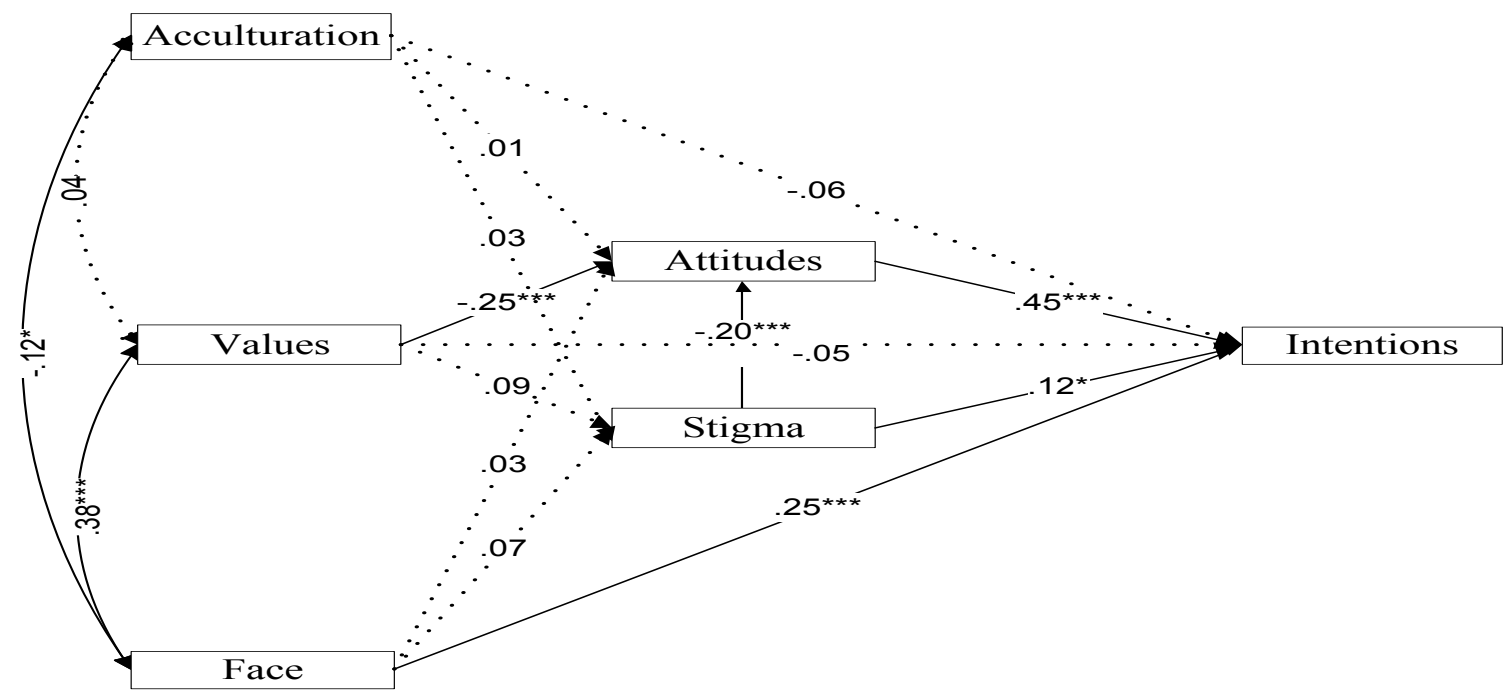

Note. $N=295$. Solid arrows represent statistically significant effects. Dashed arrows represent non-significant effects.

$*<.05 ; * *<.01 ; * * *<.001$ 
Figure 4.3 Fully Mediated TRA Model

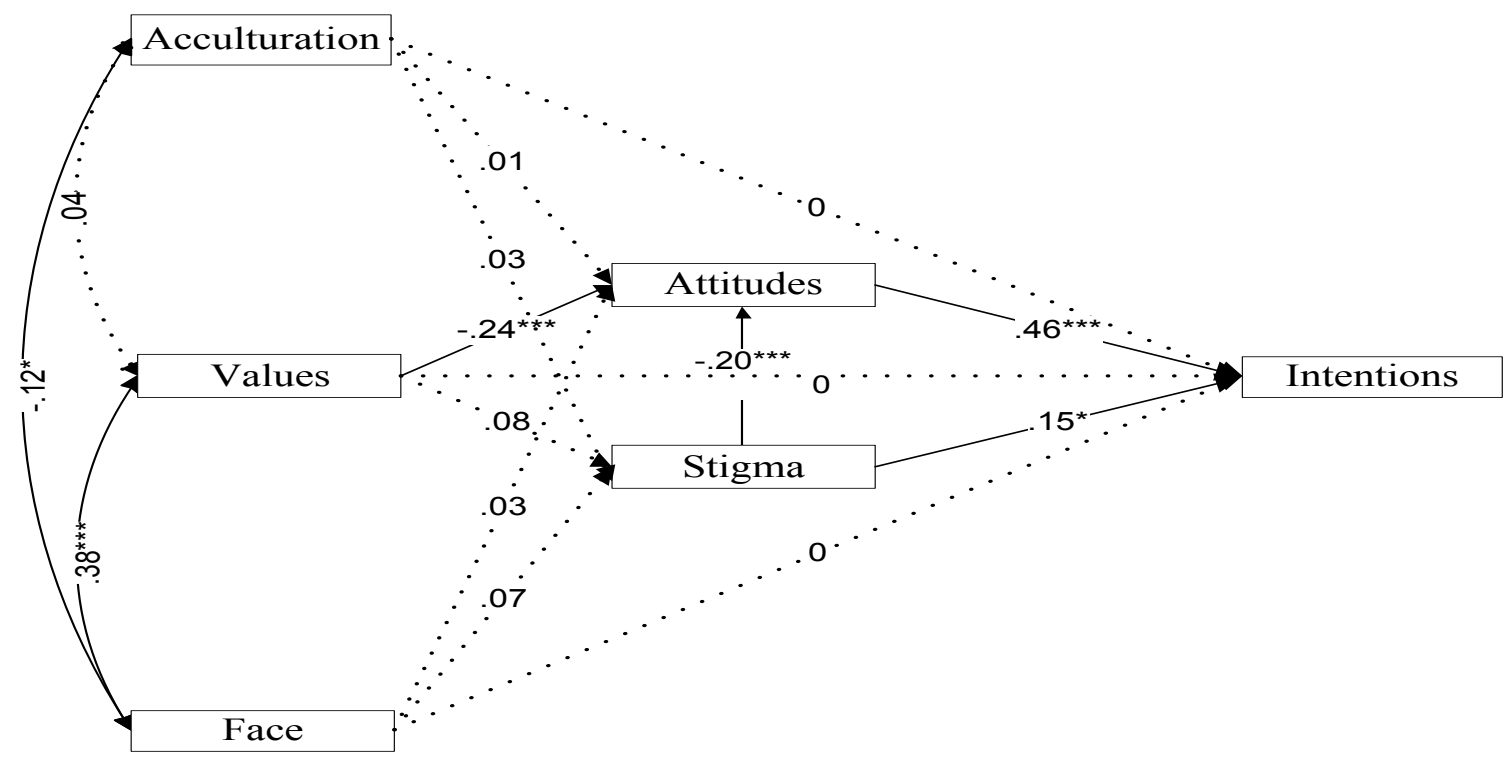

Note. $N=295$. Solid arrows represent statistically significant effects. Dashed arrows represent non-significant effects.

$*<.05 ; * *<.01 ; * * *<.001$ 
Upon path estimation, the three competing models were compared using a chisquare difference test (Kline, 2005). Furthermore, because the chi-square statistic is usually significant with large sample sizes, the ratio between the chi-square value and the model degrees of freedom also was used to determine overall data fit. Specifically, a chisquare to degrees of freedom ratio of less than 3.0 (i.e., $\chi^{2} / d f<3.00$ ) has been recommended as an indicator of a good model fit (Kline, 2005). Additional fit indices, such as the comparative fit index (CFI; .95 or greater), the root-mean-square error of approximation (RMSEA; .06 or less), and the standardized root-mean-square residual (SRMR; .08 or less) were used to evaluate the overall degree of model fit (Hu \& Bentler, 1999; Weston \& Gore, 2006). Finally, model fit also was determined by comparing the magnitude and direction of the hypothesized effects and the proportion of variance accounted for by each model (Weston \& Gore, 2006). These model comparisons were then used to evaluate Hypotheses 3a and $3 b$.

Under the criteria described above, the Non-Mediated Model fit the data poorly. As seen in Table 4.2, the chi-square statistic was significant, $\chi^{2}(10, N=295)=94.63, p$ $<.001, \chi^{2} / d f=9.46$, and the remaining indices all indicated poor model fit $(\mathrm{CFI}=.44$; RSMEA $=.17$; SRMR $=.12$ ). Furthermore, the Non-Mediated Model accounted for only $7 \%$ of the variance in help-seeking intentions. In comparison, the Partially Mediated Model provided a substantially improved chi-square statistic, $\chi^{2}=(2, N=295)=3.26$, $n s, \chi^{2} / d f=1.63$, with the remaining indices suggesting a well-fitting model $(\mathrm{CFI}=.99$; RSMEA $=.05$; and SRMR $=.01)$. In addition, the Partially Mediated Model accounted for $25 \%$ of the variance in help-seeking intentions. Finally, the Fully Mediated Model offered poor to adequate fit. The chi-square statistic was significant $\chi^{2}(5, N=295)=$ 
27.66, $p<.001, \chi^{2} / d f=5.53$, and only the SMRS met established recommendations for adequate fit $(\mathrm{CFI}=.85 ; \mathrm{RSMEA}=.12 ; \mathrm{SRMR}=.06)$. Furthermore, the Fully Mediated Model accounted for $20 \%$ of the variance in help-seeking intentions (see Table 4.3). In order to further compare the Non-Mediated, Partially Mediated, and Fully Mediated Models, a chi-square difference test was performed using recommendations offered by Weston and Gore (2006). Using this analysis, the Partially Mediated Model provided a superior data fit as compared to the Non-Mediated Model, $\Delta \chi^{2}(8, N=295)=$ 91.37, $p<.001$. Similarly, the Partially Mediated Model was superior to the Fully Mediated Model, $\Delta \chi^{2}=(3, N=295)=24.40, p<.001$, indicating that the Partially Mediated Model offered the best overall data fit, as compared to the two alternative models. Finally, the Fully Mediated Model was statistically superior to the Non-Mediated Model $\Delta \chi^{2}(5, N=295)=66.97, p<.001$, indicating that the Non-Mediated Model offered the worst data fit, as compared to the remaining two competing models.

The analyses presented above supported Hypothesis 3a, such that the Partially Mediated Model emerged as statistically superior to the Non-Mediated Model. In contrast, Hypothesis $3 \mathrm{~b}$ was only partially supported. Specifically, the Fully Mediated Model was statistically superior when compared to the Non-Mediated Model, but not when compared to the Partially Mediated Model. In fact, the Partially Mediated Model offered the best overall fit to the current data. Given the statistical superiority of the Partially Mediated Model, it was retained as the final TRA model utilized in this study. 
Table 4.2 Comparisons of the Hypothesized TRA Models

\begin{tabular}{lcccccccc}
\hline Model & $\chi^{2}$ & $\boldsymbol{d} \boldsymbol{f}$ & $\chi^{2} / \boldsymbol{d f}$ & $\boldsymbol{p}$ & CFI & RSMEA & SRMR & $\boldsymbol{R}^{2}$ \\
\hline Non-Mediated Model & 94.63 & 10 & 9.46 & $<.001$ & .44 & .17 & .12 & .07 \\
Partially Mediated Model & 3.26 & 2 & 1.63 & $>.05$ & .99 & .05 & .01 & .25 \\
Fully Mediated Model & 27.66 & 5 & 5.53 & $<.001$ & .85 & .12 & .06 & .20 \\
\hline Note. $N=295$ & & & & & & &
\end{tabular}


After retaining the Partially Mediated Model, the magnitude and directionality of each path estimate were examined for consistency with the empirical literature and the TRA (Ajzen \& Fishbein, 1980; Fishbein \& Ajzen, 2009). Consistent with prior research and the TRA, counseling attitudes emerged as a significant and unique predictor of helpseeking intentions $(\beta=.45, p<.001)$. Similarly, stigma concerns and counseling attitudes were significantly and negatively associated with each other, which also was consistent with the existing literature $(\beta=-.20, p<.001)$. Finally, stigma concerns predicted helpseeking intentions; however, the direction of the relationship was inconsistent with prior research and the TRA $(\beta=.12, p<.05)$. Specifically, stigma concerns were expected to be negatively, rather than positively, associated with help-seeking intentions.

Consistent with prior research, participants' acculturation was significantly and negatively associated with their loss of face concerns $(\beta=-.12, p<.05)$. However, contrary to prior research, acculturation was not significantly associated with the endorsement of Asian values $(\beta=.04, p<.05)$. In addition, contrary to prior research and the TRA, behavioral acculturation was not significantly associated with any of the helpseeking constructs explored in this study: help-seeking intentions $(\beta=-.06, p>.05)$, counseling attitudes $(\beta=.01, p>.05)$, and social stigma concerns $(\beta=.03, p<.05)$.

Consistent with prior research, participants' endorsement of Asian values was significantly and positively associated with loss of face concerns $(\beta=.38, p<.001)$. Similarly, Asian values were significantly and negatively related to counseling attitudes $(\beta=-.25, p<.001)$, a relationship which is consistent with the literature. However, the endorsement of Asian values was not significantly associated with participants' 
acculturation $(\beta=.04, p<.05)$. Furthermore, values failed to predict respondents' helpseeking intentions $(\beta=-.05, p>.05)$ and stigma concerns $(\beta=.09, p>.05)$.

Consistent with prior research, loss of face concerns were significantly and positively correlated with the endorsement of Asian values $(\beta=.38, p<.001)$. Furthermore, loss of face concerns were significantly and negatively associated with acculturation $(\beta=-.12, p<.05)$. Participants' loss of face concerns also were significantly associated with their intentions to seek counseling $(\beta=.35, p<.001)$. However, the direction of the relationship was inconsistent with previous research. Specifically, prior research has conceptualized loss of face concerns as a barrier, rather than a facilitator, to Asian and Asian Americans' help-seeking. Finally, contrary to previous findings and the TRA, loss of face concerns were not significantly associated with counseling attitudes $(\beta=.03, p>.05)$ or stigma concerns $(\beta=.07, p>.05)$.

\section{Testing the Hypothesized Indirect Effects}

Finally, Hypothesis $3 \mathrm{c}$ was tested by examining the statistical significance of the indirect effects specified in the Partially Mediated Model. It was hypothesized that acculturation, Asian values, and loss of face would have significant indirect effects on help-seeking intentions through the two mediators of counseling attitudes and stigma concerns. In addition, it was predicted that stigma concerns would also influence intentions indirectly through the mediating effect of counseling attitudes.

In order to test Hypothesis $3 \mathrm{c}$, the indirect effects listed above were evaluated using the bootstrap procedure described by Shrout and Bolger (2002). The first step of the analysis was to create 10,000 bootstrap samples from the original data set, using the procedure of random sampling with replacement. The Partially Mediated Model was then 
run once with each bootstrap sample to yield 10,000 estimations of each path coefficient for the specified indirect effects. Finally, the $95 \%$ confidence intervals for the obtained indirect effect estimates were examined in order to determine their statistical significance. If the $95 \%$ confidence interval did not include zero, it was concluded that the mediated effect was statistically significant at the $p=.05$ level (Shrout \& Bolger, 2002).

Based on these criteria, only two indirect effects reached statistical significance (see Table 4.3). Specifically, counseling attitudes emerged as a significant mediator in the relationship between Asian values and intentions to seek counseling $(\beta=-.10$, S.E. $=$ .03 ), with a $95 \%$ confidence interval of -.18 (lower end) and -.05 (upper end). Similarly, counseling attitudes emerged as a significant mediator of the relationship between stigma concerns and intentions to seek counseling $(\beta=-.09$, S.E. $=.03)$, with a $95 \%$ confidence interval of -.15 (lower end) to -.04 (upper end). However, none of the other indirect effects reached statistical significance in the bootstrapping analysis, thus indicating only partial support for the hypothesized mediation effects. These findings are consistent with the significant paths yielded by the Partially Mediated Model path analysis. 
Table 4.3 Bootstrap Analysis of Indirect Effects

\begin{tabular}{|c|c|c|c|c|c|}
\hline Independent & Mediator & Dependent & Indirect & S.E. ( $\beta$ ) & $95 \%$ CI \\
\hline Variable & Variable & Variable & Effect $(\beta)$ & & \\
\hline Acculturation $\rightarrow$ & Attitudes $\rightarrow$ & Intentions & .00 & .02 & $-.04, .04$ \\
\hline Acculturation $\rightarrow$ & Stigma $\rightarrow$ & Intentions & .00 & .01 & $-.01, \quad .01$ \\
\hline Asian Values $\rightarrow$ & Attitudes $\rightarrow$ & Intentions & $-.10 * *$ & .03 & $-.18,-.05^{* *}$ \\
\hline Asian Values $\rightarrow$ & Stigma $\rightarrow$ & Intentions & .00 & .01 & $-.01, \quad .03$ \\
\hline Loss of Face $\rightarrow$ & Attitudes $\rightarrow$ & Intentions & .01 & .02 & $-.04, \quad .06$ \\
\hline Loss of Face $\rightarrow$ & Stigma $\rightarrow$ & Intentions & .00 & .01 & $-.01, \quad .03$ \\
\hline Stigma $\rightarrow$ & Attitudes $\rightarrow$ & Intentions & $-.09 * *$ & .03 & $-.15,-.04 * *$ \\
\hline
\end{tabular}




\section{CHAPTER V \\ DISCUSSION}

This chapter discusses the findings of the present study by summarizing the proposed hypotheses, explaining the statistical results, outlining the strengths and limitations of the research design, providing recommendations for future empirical inquiry, and discussing relevant implications for clinical practice.

\section{Summary of Hypotheses and Research Findings}

Prior research has identified a number of cognitive and cultural predictors of Asian international students' intentions to seek counseling. For example, counseling attitudes and stigma concerns have been identified as important cognitive predictors of Asian American and Asian international students' help-seeking intentions (Heggins \& Jackson, 2003; Johnson \& Sandhu, 2007; Mori, 2000). In addition, acculturation, Asian values, and loss of face concerns also have emerged as important predictors of helpseeking among these populations (Ibrahim \& Ingram, 2007; Johnson \& Sandhu, 2007; Sheu \& Fukuyama, 2007). The purpose of this study was to determine if the cognitive and cultural predictors listed above can be integrated using the Theory of Reasoned Action (TRA, Ajzen \& Fishbein, 1980; Fishbein \& Ajzen, 2009). Three sets of hypotheses were derived based on the premises of the TRA and prior research (see Table 5.1). These hypotheses were tested by examining the bivariate associations between the 
variables of interest, using path analysis to compare relative model fit, and utilizing bootstrap analyses to test the significance of the hypothesized mediation effects.

Table 5.1 List of Study Hypotheses

\section{Hypothesis 1: Counseling attitudes and stigma concerns will emerge as significant} predictors of help-seeking intentions.

Hypothesis 1a (supported): Counseling attitudes will be positively associated with help-seeking intentions.

Hypothesis $1 \mathrm{~b}$ (not supported): Stigma concerns will be negatively associated with help-seeking intentions.

Hypothesis 1c (supported): Counseling attitudes and stigma concerns will be inversely related to each other.

Hypothesis 2: Cultural variables will emerge as significant predictors of the three help-seeking constructs (i.e., attitudes, stigma concerns, and intentions).

Hypothesis 2a (not supported): Acculturation will be negatively associated with stigma concerns and positively associated with attitudes and intentions.

Hypothesis $2 b$ (partially supported): Asian values will be positively associated with stigma concerns and negatively associated with attitudes and intentions.

Hypothesis 2c (not supported): Loss of face will be positively associated with sigma concerns and negatively associated with attitudes and intentions.

Hypothesis 3: The relationships between cultural variables and help-seeking intentions will be mediated by attitudes and stigma concerns.

Hypothesis 3a (supported): A partially mediated TRA model will offer better data fit than a non-mediated model.

Hypothesis 3b (not supported): A fully mediated TRA model also will provide a good data fit, while offering greater parsimony than the non-mediated and the partially mediated models.

Hypothesis 3c (supported): Bootstrapping analyses will support the statistical significance of the proposed indirect effects. 
The analyses used in the present study provided partial support for Hypothesis 1. Specifically, Hypothesis 1a was supported, such that Asian international students who reported more positive counseling attitudes also held greater help-seeking intentions (Hypothesis 1a). Similarly, there was support for Hypothesis 1c, such that participants who expressed greater stigma concerns also reported less positive counseling attitudes. However, Hypothesis $1 \mathrm{~b}$ was not supported, such that greater stigma concerns were associated with significantly stronger, as opposed to weaker, help-seeking intentions.

In addition, the findings of the present study provided partial support for Hypothesis 2. Specifically, Hypothesis $2 \mathrm{~b}$ was supported, such that Asian international students who reported more traditional cultural values endorsed less positive counseling attitudes and weaker help-seeking intentions. However, Hypothesis 2a was not supported, such that participants' acculturation to American society was not significantly related to their counseling attitudes, stigma concerns, or help-seeking intentions. Furthermore, Hypothesis 2c was not supported either, such that students' loss of face concerns were associated with significantly greater, as opposed to weaker, help-seeking intentions.

Finally, the findings of the current study provided partial support for Hypothesis 3. Specifically, Hypothesis 3a was supported, such that the partially mediated model offered a better data fit than the non-mediated model. Similarly, Hypothesis $3 \mathrm{c}$ also was supported, such that participants' counseling attitudes emerged as a significant mediator in the relationships between Asian values, stigma concerns, and help-seeking intentions. However, Hypothesis $3 \mathrm{~b}$ was not supported, given that the partially mediated model offered a superior fit to the fully mediated TRA model. 
These results reveal a complex pattern of relationships, suggesting that the interplay of cognitive and cultural variables examined in this study may be more complex than previously understood. Specifically, while some of the present findings are consistent with past theory and research, others run counter to the existing literature. In describing this complex array of findings, I will first outline those results which are in line with prior theory and research, followed by a discussion of inconsistent or counterintuitive findings. I will then conclude this section by outlining the implications of the present study for understanding Asian international students' help-seeking and determining the applicability of the TRA to this population.

Several of the current findings are consistent with existing research and the TRA. First, negative counseling attitudes emerged as a potential barrier to help-seeking among Asian international students. This relationship is in line with prior research (Komiya \& Eells, 2001; Liao et al., 2005; Tedeschi \& Willis, 1993; Yoon \& Jepsen, 2008; Zhang \& Dixon, 2003) and the TRA (Ajzen \& Fishbein, 1980; Fishbein \& Ajzen, 2009). In fact, Fishbein and Ajzen suggested that attitudes are the strongest available predictor of individuals' intentions, a hypothesis which is in agreement with the findings of the present study. In addition, counseling attitudes mediated the relationship between Asian values, stigma concerns, and help-seeking intentions, which also is consistent with the TRA (Ajzen \& Fishbein, 1980; Fishbein \& Ajzen, 2009). Specifically, Asian students who reported more traditional values and greater stigma concerns also endorsed less positive counseling attitudes, which, in turn, predicted lower help-seeking intentions. As such, attitudes may be an important variable of interest in future research (Liao et al., 2005; Yoon \& Jepsen, 2008). 
Another set of consistent findings involved the association between students' endorsement of Asian values and their help-seeking intentions. Consistent with prior research, Asian international students who endorsed more traditional values reported less positive counseling attitudes and lower help-seeking intentions (Gloria et al., 2008; Kim, 2007; Kim \& Omizo, 2003; Liao et al., 2005; Shea \& Yeh, 2008). In addition, the relationship between Asian values and help-seeking was fully mediated by counseling attitudes, which is in agreement with the TRA (Ajzen \& Fishbein, 1980; Fishbein \& Ajzen, 2009). This indicates that the effect of Asian values on the help-seeking process may occur primarily through the influence of counseling attitudes (Ajzen \& Fishbein, 1980; Fishbein \& Ajzen, 2009). As such, cultural values may be an important variable to consider in terms of understanding Asian international students' help-seeking intentions.

Although the results described above were consistent with prior literature, there also were several unexpected findings. For example, the present study indicated that stigma concerns were significantly and positively associated with help-seeking intentions. This is at odds with past theory and research, which has indicated negative associations between stigma and help-seeking (Ajzen \& Fishbein, 1980; Dadfar \& Friedlander, 1982; Fishbein \& Ajzen, 2009; Tedeschi \& Willis, 1993; Yoon \& Jepsen, 2008; Zhang \& Dixon, 2003). A possible explanation for these results may be the use of a stigma measure (PSOSH; Vogel, Wade, \& Ascheman, 2009), which has never before been used with Asian international students. In addition, participants may have misread the PSOSH items, such that the scale may be assessing their fears of publicly acknowledging psychological problems. As such, students who fear stigmatization by significant others may be more likely to seek help from a mental health professional due to the confidential 
nature of counseling. Prior research provides some support for this hypothesis, suggesting that Asian international students refrain from sharing emotional problems with their families (Kindaichi et al., 2005; Mau \& Jepsen, 1990; Olivas \& Li, 2006). This interpretation, however, is speculative and needs to be explored in future research.

Another set of inconsistent results involved the effect of loss of face on the helpseeking process. The present findings indicated that loss of face concerns were associated with stronger help-seeking intentions, a finding which is at odds with existing theory and research (Chou, 2000; Zane \& Mak, 2003; Zane \& Yeh, 2002; Zayco, 2009). A possible explanation for this inconsistency may be that counseling does not entail loss of face for Asian international students if it can be kept confidential. Based on my own clinical experience, Asian international students rarely tell their families that they are seeking counseling. In fact, because of their geographic separation, it may be easier for international students to conceal their use of counseling from significant others back home. Furthermore, loss of face may be associated with greater distress and more acculturation difficulties for Asian international students, both of which are predictive of greater help-seeking (Cepeda-Benito \& Short, 1998; Cramer, 1999; Liao, Rounds, \& Klein, 2005). Thus, the relationship between loss of face and Asian international students' help-seeking may be more complex than previously anticipated.

A final set of inconsistent findings involved the relationship between behavioral acculturation and help-seeking intentions. Specifically, the present study indicated that acculturation was not significantly associated with Asian international students' counseling attitudes, stigma concerns, or help-seeking intentions. This is inconsistent with prior research, which found a positive association between acculturation and help- 
seeking among Asian American and Asian international college students (Atkinson \& Gim, 1989; Atkinson et al., 1995; Frey \& Roysicar, 2006; Liao et al., 2005; Tata \& Leong, 1994; Zhang \& Dixon, 2003). A possible explanation for these results is that the present study used a newly developed bidimensional acculturation scale (AAMAS-M; Chung et al., 2004) instead of the unidimensional SL-ASIA (Suinn et al., 1992; Suinn et al., 1987). Furthermore, the present study used only the AAMAS-EA (acculturation) subscale, but did not include the corresponding AAMAS-CO (enculturation) subscale. In fact, enculturation may be a better predictor of Asian international students' help-seeking, given that the process of seeking counseling is not a common practice in many Asian cultures. This hypothesis, however, needs to be explored in future research.

Overall, the present study suggested that counseling attitudes and cultural values may impede Asian international students' help-seeking. On the other hand, fear of being stigmatized by significant others and/or losing face may actually facilitate seeking help from mental health professionals, possibly due to the confidential nature of the counseling relationship. As such, the pattern of relationships among stigma, loss of face, and help-seeking may be more complex than previously anticipated. Furthermore, the present study indicated that the TRA may provide a starting point for understanding Asian international students' help-seeking. Specifically, counseling attitudes emerged as a mediator between cultural values and help-seeking intentions, which is consistent with the TRA. However, several TRA propositions were not supported, such that stigma concerns did not emerge as a significant mediator. Furthermore, the fully mediated model did not fit the data well, indicating that the TRA may offer an oversimplified theoretical model for this population. As such, the TRA may need to be extended in future research 
in order to more accurately reflect the complexity of the help-seeking process (Bayer \& Peay, 1997; Halgin et al., 1985; Halgin et al., 1987; Vogel, et al., 2005).

\section{Study Strengths and Limitations}

Given the above findings, it is clear that the present study possesses a number of strengths and limitations. A particular strength was integrating a variety of cognitive and cultural predictors of help-seeking. Specifically, previous studies have tended to examine counseling attitudes, stigma concerns, and help-seeking intentions in isolation, thus failing to consider complex multivariate effects (Komiya \& Eells, 2001; Liao et al., 2005; Tedeschi \& Willis, 1993; Yoon \& Jepsen, 2008; Zhang \& Dixon, 2003). Furthermore, prior research also has examined acculturation, Asian values, and loss of face concerns individually, while failing to integrate all three predictors into a conceptual model (Gloria et al., 2008; Kim, 2007; Kim \& Omizo, 2003; Liao et al., 2005; Shea \& Yeh, 2008). A third strength was using the TRA as an integrative theoretical framework. Much of the existing help-seeking literature is atheoretical in nature, highlighting the need to develop and test alternative theoretical frameworks (Vogel, et al., 2005).

This study also contributed to the literature by examining Asian international students as a population in its own right (Yoon \& Portman, 2009). In fact, much of the existing literature has tended to combine Asian international students with foreign students from other geographic regions while failing to explore potential cultural differences (Anderson \& Myer, 1985; Mitchell et al., 2007; Nilsson et al., 2004; Yakushko et al., 2008). Another problematic trend has been to clump foreign-born Asian international students together with U.S.-born Asian American students, thus obscuring possible differences based on immigrant status, acculturation level, and adjustment 
experiences in the U.S. (Atkinson \& Gim, 1989; Gloria et al., 2008; Gong et al., 2003; Kim, 2007; Miville \& Constantine, 2007; Shea \& Yeh, 2007; Tata \& Leung, 1994; Zayco, 2009). The present study attempted to avoid both of these problems by focusing specifically on Asian international students and screening out participants who did not meet the eligibility requirements for country of origin or international student status.

Despite its strengths, the current study suffered from a number of limitations. One limitation was the use of questionnaires that were developed with Asian American, rather than Asian international, populations. Specifically, the measures used to assess acculturation (AAMAS-EA; Chung, Kim, \& Abreu, 2004), Asian values (AAVS-M; Kim, Li, and Ng, 2005), and loss of face (LOF, Zane \& Yeh, 2002) were all validated with Asian American college students, raising a concern about their applicability to an international population. Specifically, it is possible that the process of acculturation occurs differently for Asian international students, who can be defined as first-generation sojourners in the U.S., as compared to U.S. - born Asian Americans, who are typically second, third, or fourth generation immigrants in the U.S. (Sodowsky \& Plake, 1992).

Furthermore, the acculturation and loss of face scales used in the present study assessed general interpersonal behaviors, but did not relate to counseling specifically. For example, the AAMAS-EA consisted of items assessing frequency of interaction with American peers, frequency of viewing American media, preference for American food, and self-reported English fluency. Furthermore, the LOF assessed general interpersonal tendencies, such as keeping a low profile and avoiding public mistakes or criticism. As such, these measures did not assess cultural attitudes, values, or behaviors that were closely related to help-seeking. This may explain some of our counterintuitive or 
findings. In fact, the results may have been different if the study had incorporated a different set of measures or if it had employed subsets of items which specifically related to the help-seeking process. For example, loss of face items that related more closely to confidentiality may have had a stronger relationship to help-seeking. However, using subsets of established measures is problematic, given that there is typically little psychometric data on the use of individual items.

An additional measurement issue involves the help-seeking instruments employed in the present study (i.e., ISCI, ATSPPH-SF, and PSOSH). Specifically, there was considerable variability in the way each measure referred to the process of seeking counseling. Whereas the ISCI used the term "seek counseling," the ATSPPH-SF used the phrases "talk to a psychologist," "get professional attention," "seek psychotherapy," and "get psychological help." Finally, the PSOSH asked participants to imagine that they had a problem that "needed to be treated by a mental health professional" and then imagine how significant others would respond if the participant "sought mental health services." This wide variability in terminology may have been confusing to participants, especially given that many of them may have limited knowledge about counseling (Ibrahim \& Ingram, 2007; Johnson \& Sandhu, 2007). As such, participants may have been confused about the meaning of terms such as "counseling," "psychotherapy," and "mental health services" and might have felt unsure how to respond. As such, one significant limitation of the present study was the failure to define the term "counseling" and to use a consistent terminology across the entire survey.

A final measurement concern involves the potential for participants to misunderstand survey items due to an existing language barrier. Unfortunately, this study 
failed to incorporate a formal assessment of respondents' English fluency beyond the inclusion of three validation items that were geared to detect random responding (Wei, Heppner, Mallen, Ku, Liao, \& Wu, 2007). Finally, given some of the counterintuitive findings of the present study, it would have been helpful to include open-ended questions collecting qualitative responses. For example, open-ended questions could better assess participants' fears of disclosing psychological problems to significant others. Such qualitative data could then facilitate the interpretation of the obtained quantitative results.

An additional limitation involved the use of convenience sampling, given that participants were recruited from U.S. universities with large international student populations. In addition, recruitment occurred with the help of student organization officers who distributed an e-mail survey invitation. This approach may have produced a potentially unrepresentative sample, given that all participants were members of international student organizations at large urban universities. As such, international students who were not members of an ethnic or a cultural student organization or who attended small universities in rural areas could not be sampled in the study. In addition, because of this recruitment approach, it was difficult to estimate how many international students chose to disregard the e-mail invitation. As such, there is no available data about overall survey response rate. Finally, a large number of participants $(17.4 \%, n=103)$ failed to complete the survey, signaling the possibility of selective attrition. However, the preliminary statistical analyses found no significant differences between survey completers and non-completers on any of the demographic variables of interest.

Yet another limitation was combining international students from East, Southeast, South, and Central Asia, while obscuring potential cultural, historical, linguistic, and 
religious differences. Specifically, it is likely that international students coming from countries as diverse as Japan, China, India, Pakistan, and the Philippines may represent diverse cultural backgrounds (Ibrahim \& Ingram, 2007; Johnson \& Sandhu, 2007; Sheu \& Fukuyama, 2007). Many of these differences may be obscured by combining participants into a single "Asian" category. For example, the relationships between cultural values and help-seeking may differ based on country of origin. This hypothesis, however, could not be examined, given that all participants were combined in a single sample. Collapsing participants across diverse national groups is a common problem in the literature on Asian American and Asian international populations. In fact, given the large number of Asian countries and the minority status of Asian American and Asian international students in the U.S., collapsing participants across country of origin is often seen as necessary in order to recruit large enough samples (Yoon \& Portman, 2009). Another limitation of the present study involved the inability to demonstrate cause-andeffect relationships (Ajzen \& Fishbein, 1980; Fishbein \& Ajzen, 2009). Specifically, participants' endorsement of Asian values was hypothesized to cause less positive counseling attitudes, leading to lower help-seeking intentions. These cause-and-effect propositions, however, could not be empirically supported by the cross-sectional, correlational methods employed in the current study. None of the variables in this study was manipulated using an experimental design, precluding the ability to demonstrate cause-and-effect (Tabachnick \& Fidell, 2007; Kline, 2005). Furthermore, it should be noted that the current study assessed participants' self-reported intentions to seek counseling rather than their actual help-seeking behavior. Although the TRA has suggested that intentions are a reliable predictor of behavior, others have argued that 
intentions do not always lead to action (Romano \& Netland, 2008). As such, the present findings may not necessarily generalize to actual help-seeking behavior.

A final limitation is the choice of the TRA as a conceptual framework. Although the use of theory is a significant strength of this study, the TRA itself has been criticized as an oversimplified model of human decision-making (Romano \& Netland, 2008). One limitation of the TRA is that it conceptualizes behavior as rational and predictable, which is not always the case (Ajzen \& Fishbein, 1980; Fishbein \& Ajzen, 2009). Furthermore, the TRA emphasizes cognitive variables while minimizing the influence of emotion. For example, one factor that often drives people to seek counseling is the experience of psychological distress (Cepeda-Benito \& Short, 1998; Cramer, 1999; Liao et al., 2005). By excluding this affective predictor, the TRA may fail to explain an important aspect of help-seeking. Yet another criticism is that the TRA ignores potential barriers that can intervene between intentions and behavior (Fishbein \& Ajzen, 2009; Romano \& Netland, 2008). For example, a person may intend to seek counseling but may be unable to do so because of lack of transportation, access to services, or financial resources. Other potential limitations of the TRA include failure to consider factors that facilitate helpseeking, such as therapists' multicultural competence and the mental health agency's overall commitment to issues of diversity. Thus, future research needs to address the limitations of the TRA, in addition to testing alternative help-seeking frameworks.

\section{Implications for Future Research}

Based on the findings and limitations discussed above, several recommendations can be made for future inquiry. One direction would be to clarify some of the inconsistent findings reported in the present study. A second direction would be to correct some of the 
methodological limitations inherent in the current research. Specifically, future investigations might address current empirical limitations, such as using questionnaires that were not validated with this population, using measures that did not employ a consistent help-seeking terminology, combining students from different countries into one sample, failing to demonstrate cause-and-effect, and utilizing a potentially oversimplified theoretical framework. This section will discuss each of these opportunities for future research, starting with the clarification of inconsistent findings.

One way in which future investigations can illuminate the findings of the present study would be to examine the complex relationships among stigma, loss of face, and help-seeking. Contrary to existing research, stigma and loss of face seemed to facilitate, rather than impede, seeking help from mental health professionals. One possible explanation is that Asian international students may be afraid to disclose psychological problems to significant others due to fears of disappointing their friends and families back home. As such, counseling may allow students to receive help without disclosing problems to their friends and families. Future research could explore this hypothesis by assessing intentions to seek help from significant others in addition to mental health professionals. Furthermore, future inquiry could employ qualitative methods, which can provide in-depth understanding of subjective experiences and cultural views (Hanson, Creswell, Clark, et al., 2005; Haverkamp, Morrow, \& Ponterotto, 2005). As such, qualitative research may be uniquely suited to illuminate participants' stigma and loss of face concerns in the context of seeking professional and non-professional help.

A second way in which future research can clarify the current findings would be to examine the relative influence of acculturation and enculturation on the help-seeking 
process. Specifically, in the present study, acculturation failed to predict any of the helpseeking constructs of interest, suggesting that it was unrelated to Asian international students' counseling attitudes, stigma concerns, and help-seeking intentions. It is possible, however, that the alternative construct of enculturation may be a stronger predictor of help-seeking in this population. To examine this hypothesis, future research could include measures of both acculturation and enculturation. By doing so, researchers can elucidate the relative influence of acculturation and enculturation on the help-seeking process. Furthermore, this line of inquiry could advance researchers' understanding of the acculturation-enculturation process, given that most of the existing literature is based on a unidimensional understanding of these constructs (Chung, Kim, \& Abreu, 2004).

Beyond clarifying the present findings, future research can correct some of the limitations of the current design, beginning with measurement issues. One such issue included the use of acculturation, Asian values, and loss of face scales which were never validated with an Asian international sample. In addition, the acculturation and loss of face scales assessed global behaviors that did not necessarily relate to help-seeking. Furthermore, the help-seeking measures used in the survey used varied and inconsistent terminology, while failing to provide a clear definition of counseling. This lack of consistency in terminology could be confusing for international students who typically know little about counseling. Finally, the present study failed to include a formal assessment of participants' English fluency, raising the possibility that some respondents may have misunderstood the questionnaire items. Given these limitations, future research needs to validate the present scales with Asian international students, in addition to including more targeted measures which relate directly to the help-seeking process. In 
addition, future research needs to use clear terminology and provide a consistent definition of counseling. Finally, researchers need to routinely assess participants' English fluency in order to determine respondents' level of survey comprehension. This could minimize potential misunderstandings and ensure the accuracy of participant data. Another significant limitation was combining international students from diverse countries of origin into a single sample. Future research could compensate for this limitation by conducting detailed statistical analyses that examine within-group differences based on country of origin (Yoon \& Portman, 2009). To do that, however, researchers would need to recruit large samples of international students to obtain sufficient $n$-sizes to conduct these analyses (Tabachnick \& Fidell, 2007). An alternative approach would be to collapse students across geographic regions (e.g., South and East Asia) instead of examining individual countries of origin. Thus, researchers should employ a variety of approaches in order to examine the cultural diversity existing among Asian international students as a population (Yoon \& Portman, 2009).

Future research also can use experimental and longitudinal designs to elucidate the cause-and-effect relationships that were implied in the present study (Tabachnick \& Fidell, 2007). For example, researchers could prime participants' endorsement of traditional cultural values to determine if such priming activates more negative counseling attitudes. Researchers also could use longitudinal studies to track changes in participants' acculturation, Asian values, loss of face concerns, and help-seeking as they occur over time. For example, researchers could assess possible acculturation changes and determine if these are accompanied by corresponding shifts in counseling attitudes. Conducting longitudinal research may be especially relevant given that international 
students encounter numerous developmental and cultural transitions (Johnson \& Sandhu, 2002; Yoon \& Portman, 2009). Conducting such research, however, may be challenging, given the difficulty of recruiting large international student samples and the accompanying problem of high participant attrition (Kaczmarek, Matlock, Merta, Ames, \& Ross, 1995; Toyokawa \& Toyokawa, 2002; Ying, 2005).

Finally, future research could modify the TRA in order to address some of its limitations, or, alternatively, test new and improved theoretical frameworks. For example, a modified TRA needs to incorporate affective predictors of help-seeking, such as level of psychological distress and negative affect, given that both factors have been associated with greater help-seeking (Cramer, 1999; Liao, Rounds, \& Klein, 2005). Another way in which the TRA could be modified includes incorporating external supports and barriers to the help-seeking process. For example, possible supports may include the availability of low-cost and accessible mental health services, the availability of therapists who are knowledgeable about Asian cultural values and Asian international students' presenting concerns, and the availability of counselors who can provide services in the client's native language (Johnson \& Sandhu, 2007; Sheu \& Fukuyama, 2007). On the other hand, possible barriers may include lack of knowledge about counseling services, lack of financial means or transportation to access counseling, and the lack of culturally competent mental health providers who can work with Asian international students (Ibrahim \& Ingram, 2007). Finally, future research needs to move beyond predicting help-seeking intentions and, instead, assess actual behavior. Predicting behavior is the ultimate goal of the TRA, so researchers who utilize this framework would need to also assess actual help-seeking behavior (Fishbein \& Ajzen, 2009; Romano \& Netland, 2008). 
Beyond modifying and extending the TRA, future research can also focus on examining alternative theoretical frameworks which can also incorporate cognitive, behavioral, affective, and cultural predictors of the help-seeking process. One alternative framework that may be tested in future research is Cramer's (1999) help-seeking model, which considers affect (i.e., psychological distress) as an important predictor of helpseeking behavior (Liao, Rounds, \& Klein, 2005). This model includes psychological distress, social support, counseling attitudes, and help-seeking intentions as predictors of counseling service utilization. In addition, Cramer's theory can be extended to include cultural variables, as demonstrated by Liao, Rounds, and Klein (2005) who supplemented the model by adding acculturation and Asian values as predictors of Asian American students' help-seeking intentions. Another option would be to utilize the Theory of Planned Behavior (TPB), a recent modification of the TRA, which takes into account external barriers intervening between intentions and behavior (Fishbein \& Ajzen, 2009; Romano \& Netland, 2008). The TPB can further be modified by including factors that support the help-seeking process, such as the availability of affordable, accessible, and multiculturally competent counseling providers. By incorporating these modifications, the TPB parallels other well-established psychological theories which include both supports and barriers to human decision making (e.g., Social Cognitive Career Theory; Lent, Brown, \& Hackett, 1996). As such, future research can test a variety of new and revised theoretical models in order to account for Asian international students' helpseeking process. 


\section{Implications for Clinical Practice}

Apart from informing future research, the present study also could have practical implications for mental health practitioners. The current findings may be particularly relevant to university counseling center professionals, given that they are most likely to provide assessment, consultation, counseling, outreach, and other mental health services to Asian international students (Lin \& Pedersen, 2007; Mori, 2000; Tidwell \& Hanassab, 2007). Specifically, the findings of the present study can inform the ways in which counseling center staff advertise their services to international students, the types of outreach programs they develop for this population, and the selection of specific interventions during the process of individual counseling. Counseling center staff may also be in a unique position to conduct multicultural needs assessments of international student concerns, thus further incorporating the findings of the present study into service delivery (Leong \& Chou, 1996; Pedersen, 1991; Prieto, 1995).

In terms of clinical practice, university counseling staff may need to be aware that some Asian international students might hold negative counseling attitudes (Ibrahim \& Ingram, 2007; Sheu \& Fukuyama, 2007; Wang, Lin, Pang, \& Shen, 2007). The importance of examining Asian international students' attitudes toward seeking psychological help was supported by the findings of the present study, which indicated that participants who reported more negative attitudes were less likely to indicate that they would seek counseling in the future. As such, mental health professionals might need to use advertising approaches and outreach presentations that promote positive counseling attitudes among Asian international students. One way in which this could be achieved is by presenting counseling as an adaptive way of dealing with the adjustment 
and acculturation stressors experienced by international students. Another way of promoting positive counseling attitudes among Asian international students might be to provide culturally competent counseling and, if possible, to offer services in a variety of Asian languages, including Japanese, Korean, Hindi, Cantonese, and Mandarin Chinese. Furthermore, the present findings suggested that traditional cultural values could discourage professional mental health help-seeking, thus resulting in negative counseling attitudes and low help-seeking intentions among Asian international students. As such, counseling center professionals may need to grapple with the difficult question of addressing cultural value conflicts in the context of working with Asian international clients. Specifically, Sue and Sue (2008) have suggested that Western-based models of counseling traditionally focus on the individual and emphasize emotional expressivity, insight, self-disclosure, and direct interpersonal communication. These values could conflict with the cultural beliefs of Asian international students who are raised in cultures which emphasize collectivism, emotional self-control, protecting one's social face, and communicating in indirect and subtle ways. As such, mental health professionals would face the dilemma of modifying counseling in order to fit the values of cultural minority clients vs. trying to change client's cultural beliefs in order to fit within a Western-based counseling model (Sue \& Sue, 2008).

Although the dilemma of facing cultural value conflicts in counseling is certainly challenging, my personal experience and the findings of the present study indicate that mental health professionals may need to modify counseling in order to better serve Asian international students. Specifically, the present findings indicated that cultural value conflicts may prevent Asian international students from entering counseling in the first 
place, and as such counseling center professionals might consider modifying their interventions in order to make them more appealing and culturally appropriate for this population. In addition, most international students reside in the United States for brief periods of times, and they typically return to their home countries after completing their studies (Coppi, 2007). As such, it may be unethical to challenge or modify Asian international students' cultural beliefs, because doing so may result in greater reverse culture shock upon re-entry into the home culture (Leung, 2007).

Based on my personal counseling experience and the current professional literature, counseling center practitioners might be able to modify counseling and make it culturally relevant for Asian international students in several ways. One approach might be to advertise counseling in a way that is consistent with Asian international students' cultural worldviews (Wang, Lin, Pang, \& Shen, 2007). For example, practitioners could deemphasize the importance of emotional self-disclosure, and instead use cognitivebehavioral interventions, which could be more consistent with the Asian value of emotional self-control (Sheu \& Fukuyama, 2007). Counseling also could be framed as a proactive way to deal with problems and promote academic and career success. This could resonate with Asian international students' desire to honor their families by achieving academically and professionally (Ibrahim \& Ingram, 2007; Wang, Lin, Pang, \& Shen, 2007). In addition, counseling could be advertized as a way of achieving physical and emotional health, thus connecting with Asian students whose spiritual traditions emphasize mind-body interaction (Sheu \& Fukuyama, 2007; Sue \& Sue, 2008; Zhang \& Dixon, 2001). Finally, counseling center professionals could strive to demonstrate respect and understanding of Asian international students' values, thus 
strengthening the therapeutic relationship and their clients' likelihood to persist with counseling (Sue \& Sue, 2008; Zhang \& Dixon, 2003).

Beyond the importance of considering cultural values, another clinical implication of the present study involves addressing Asian international students' concerns with social stigma and loss of face (Brinson \& Kotler, 1995; Ibrahim \& Ingram, 2007; Sheu \& Fukuyama, 2007). Specifically, the findings of the present study indicated that some Asian international students could avoid sharing psychological problems with their significant others because of concerns with disappointing or worrying their friends and families back home. Furthermore, the present findings suggested that counseling could allow Asian international students to receive help without disclosing psychological difficulties to significant others. This may explain why, in the present study, greater stigma fears and stronger loss of face concerns were significantly associated with stronger intentions to seek counseling among Asian international students. As such, the findings of the present study indicated that university staff could promote greater counseling service utilization by emphasizing the confidential nature of the therapeutic relationship. Specifically, mental health professionals might consider emphasizing confidentiality when they advertize counseling services to Asian international students. They might also explore issues of confidentiality as part of informed consent, while reassuring their Asian international clients that their information is kept confidential within the limits set by federal and state laws and currently established professional ethics codes.

Beyond discussing the confidential nature of the counseling relationship, however, university professionals also might consider the negative repercussions for 
Asian international students who chose to conceal psychological problems from their families significant others. Specifically, Asian international students' fear of revealing personal problems could lead to increased interpersonal isolation and reduced social support, thus compounding existing psychological difficulties. To address these concerns, counseling center professionals might design outreach programs that reduce the stigma of psychological problems (Engel, Insalaco, Singaravelu, \& Kennon, 2007; Lin \& Yi, 1997). Counseling professionals also could provide psychoeducation about the importance of social support, thus encouraging help-seeking from both professional and non-professional sources (Lacina, 2002). As such, the findings of the present study suggest that by reducing the stigma of mental health illness, counseling center professionals might be able to encourage Asian international students to seek counseling in addition to seeking social support from their friends, families, and significant others.

Beyond attending to issues of counseling stigma and loss of face, practitioners might consider the potential effects of acculturation and enculturation on Asian international students' help-seeking and presenting concerns (Fernandez, 1988; Ibrahim \& Ingram, 2007; Sheu \& Fukuyama, 2007). Although this study found a non-significant effect in the present Asian international student sample, other research has reported a significant positive relationship between acculturation and help-seeking, indicating that practitioners may need to explore this issue on an individual basis (Frey \& Roysicar, 2006; Liao et al., 2005; Tata \& Leong, 1994; Zhang \& Dixon, 2003). Counseling center staff also may need to differentiate between international students' acculturation to American society and their retention of a native cultural identity (i.e., enculturation), as the two processes may occur independently (Chung et al., 2004; Miller, 2007). As such, it 
is possible that enculturation might be a stronger predictor of psychological help-seeking among Asian international students, given that the present findings indicated a nonsignificant effect for acculturation. As such, counseling center professionals might need to assess Asian international students' enculturation, in addition to considering acculturation issues in the context of seeking counseling (Johnson \& Sandhu, 2007; Kim $\&$ Abreu, 2001).

Finally, mental health professionals could consider using theory-driven interventions to promote culturally sensitive mental health services for Asian international students. Several alternative frameworks could be used to design outreach programs, psychoeducational workshops, and counseling services that are sensitive to the needs of this population. These may include a modified and extended version of the Theory of Reasoned Action (TRA, Ajzen \& Fishbein, 1980; Fishbein \& Ajzen, 2009), the newly developed Theory of Planned Behavior (TPB; Fishbein \& Ajzen, 2009; Romano \& Netland, 2008), and Cramer's help-seeking model (Crammer, 1999; Liao et al., 2005). Regardless of the selected model, practitioners likely need to integrate a range of cultural, cognitive, and affective variables, in addition to considering both facilitators and barriers of the help-seeking process (Fernandez, 1988; Ibrahim \& Ingram, 2007; Lin \& Pedersen, 2007; Khoo \& Abu-Rasain, 1994; Sheu \& Fukuyama, 2007). Beyond utilizing the theories outlined above, practitioners may be in a unique position to develop new frameworks to counseling advertisement, outreach programming, and service delivery. For example, college counseling center professionals could conduct a multicultural needs assessment of Asian international students' mental health concerns (Leong \& Chou, 
1996; Pedersen, 1991; Prieto, 1995). The results of this assessment could then be used to develop culturally sensitive services for this population.

In conclusion, I hope that the present findings could have practical implications for college counseling professionals working with Asian international students. It is important to recognize, however, that the current study suffered from a number of limitations, and, as such, mental health practitioners would need to evaluate each suggestion presented above. Furthermore, university counseling center staff may be in a unique position to develop and evaluate a variety of mental health services for Asian international students. It is hoped that such science-practice integration would promote the development of culturally sensitive interventions, improve counseling treatment outcomes, and help establish the credibility of the mental health field among the international student community. 


\section{REFERENCES}

Anderson, T. R., \& Myer, T. E. (1985). Presenting problems, counselor contacts, and "no shows": International and American college students. Journal of College Student Personnel, 26, 500-503.

Ajzen, I., \& Fishbein, M. (1980). Understanding attitudes and predicting behavior. Englewood Cliffs, NJ: Prentice Hall.

Atkinson, D. R., \& Gim, R. H. (1989). Asian-American cultural identity and attitudes toward mental health services. Journal of Counseling Psychology, 36, 209-212. doi:10.1037/0022-0167.36.2.209

Atkinson, D. R., Lowe, S., \& Matthews, L. (1995). Asian-American acculturation, gender, and willingness to seek counseling. Journal of Multicultural Counseling and Development, 23, 130-138.

Berry, J. W. (1994). Acculturation and psychological adaptation: An overview. In Bouvy, A., van de Vijver, F. J. R., and Boski, P. (Eds.) Journeys in cross-cultural psychology: An overview. (pp. 129-141). Lisse, Netherlands: Swets \& Zeitlinger Publishers.

Berry, J. W. (2003). Conceptual approaches to acculturation. In K. M. Chun, P. B. Organista, \& G. Marin (Eds.), Acculturation: Advances in theory, measurement, and applied research (pp. 17-37). Washington, DC: American Psychological Association.

Brinson, J. A., \& Kottler, J. (1995). International students in counseling: Some alternative models. Journal of College Student Psychotherapy, 9, 57-70. doi:10.1300/J035v09n03_05

Cash, T. F., Begley, P. J., McCown, D. A., \& Wiese, B. C. (1975). When counselors are heard but not seen: Initial impact of physical attractiveness. Journal of Counseling Psychology, 22, 273-279. doi:10.1037/h0076730

Cepeda-Benito, A., \& Short, P. (1998). Self-concealment, avoidance of psychological services, and perceived likelihood of seeking professional help. Journal of Counseling Psychology, 45, 58-64. doi:10.1037/0022-0167.45.1.58 
Chen, S. X., \& Mak, W. W. S. (2008). Seeking professional help: Etiology beliefs about mental illness across cultures. Journal of Counseling Psychology, 55, 442-450. doi: $10.1037 / \mathrm{a} 0012898$

Chou, E. L. (2000). Predictors of treatment acceptability, willingness to see a counselor, and counselor preferences for Asian Americans and whites: Acculturation, loss of face, self-construals, and collective self-esteem. Dissertation Abstracts International: Section B: The Sciences and Engineering, 60, 4209.

Christpher, M. S., Skillman, G. D., Kirkhart, M. W., \& D’Souza, J. B. (2006). The effect of normative and behavioral persuasion on help seeking in Thai and American college students. Journal of Multicultural Counseling and Development, 34, 8093.

Chung, R. H., Kim, B. S., \& Abreu, J. M. (2004). Asian American Multidimensional Acculturation Scale: Development, factor analysis, reliability, and validity. Cultural Diversity and Ethnic Minority Psychology, 10, 66-80. doi:10.1037/10999809.10.1.66

Constantine, M. G., Kindaichi, M., \& Okazaki, S. (2005). A qualitative investigation of the cultural adjustment experiences of Asian international college women. Cultural Diversity and Ethnic Minority Psychology, 11, 162-175. doi:10.1037/1099-9809.11.2.162

Constantine, M. G., Okazaki, S., \& Utsey, S. O. (2004). Self-concealment, social selfefficacy, acculturative stress, and depression in African, Asian, and Latin American international college students. American Journal of Orthopsychiatry, 74, 230-241. doi: 10.1037/0002-9432.74.3.230

Corrigan, P. W. (2004). How stigma interferes with mental health care. American Psychologist, 59, 614-625. doi:10.1037/0003-066X.59.7.614

Coppi, C. (2007). The changing landscape of international student advisement: Balancing bureaucracy with student advocacy. In H. Singaravelu \& M. Pope (Eds.) $A$ handbook for counseling international students in the United States (pp. 3-13). Alexandria, VA: American Counseling Association.

Cramer, K. M. (1999). Psychological antecedents to help-seeking behavior: A reanalysis using path modeling structures. Journal of Counseling Psychology, 46, 381-387. doi:10.1037/0022-0167.46.3.381

Dadfar, S., \& Friedlander, M. L. (1982). Differential attitudes of international students toward seeking professional psychological help. Journal of Counseling Psychology, 29, 335-338. doi:10.1037/0022-0167.29.3.335 
Deane, F. P., \& Chamberlaine, K. (1994). Treatment fearfulness and distress as predictors of professional psychological help-seeking. British Journal of Guidance and Counseling, 22, 207-218. doi:10.1080/03069889400760211

Elhai, J. D., Schwinle, W., \& Anderson, S. M. (2008). Reliability and validity of the Attitudes Toward Seeking Professional Psychological Help Scale - Short Form. Psychiatry Research, 159, 320-329. doi:10.1016/j.psychres.2007.04.020

Engel, M. B., Insalaco, G., Singaravelu, H. D., \& Kennon, K. (2007). Programs and outreach for international students. In H. D. Singaravelu and M. Pope, $A$ handbook for counseling international students in the United States (pp. 311316). Alexandria, VA: American Counseling Association.

Fernandez, M. S. (1988). Issues in counseling Southeast-Asian students. Journal of Multicultural Counseling and Development, 16, 157-166.

Finney, S. J., \& DiStefano, C. (2006). Nonnormal and categorical data in structural equation models. In G.R. Hancock \& R.O. Mueller (Eds.). A second course in structural equation modeling (pp. 269-314). Greenwich, CT: Information Age.

Fischer, E. H., \& Farina, A. (1995). Attitudes toward seeking professional psychological help: A shortened version and considerations for research. Journal of College Student Development, 36, 368-373.

Fischer, E. H., \& Turner, J. L. (1970). Orientations to seeking professional help: Development and research utility of an attitude scale. Journal of Consulting and Clinical Psychology, 35, 79-90. doi:10.1037/h0029636

Fishbein, M. \& Ajzen, I. (2009). Predicting and changing behavior: The reasoned action approach. New York, NY: Psychology Press.

Frazier, P. A., Tix, A. P., \& Barron, K. E. (2004). Testing moderator and mediator effects in counseling psychology research. Journal of Counseling Psychology, 51, 115134. doi: $10.1037 / 0022-0167.51 .1 .115$

Frey, L. L., \& Roysicar, G. (2006). South Asian and East Asian international students' perceived prejudice, acculturation, and frequency of help resource utilization. Journal of Multicultural Counseling and Development, 34, 208-222.

Gloria, A. M., Castellanos, J., \& Park, Y. S. (2008). Adherence to Asian cultural values and cultural fit in Korean American undergraduates' help-seeking attitudes. Journal of Counseling and Development, 86, 419-428.

Goffman, E. (1955). On face work: An analysis of ritual elements in social interaction. Psychiatry: Journal for the Study of Interpersonal Processes, 18, 213-231. 
Gong, F., Gage, S. L., \& Tacata, L. A. (2003). Help-seeking behavior among Filipino Americans: A cultural analysis of face and language. Journal of Community Psychology, 31, 469-488. doi:10.1002/jcop.10063

Halgin, R. P., Weaver, D. D., \& Donaldson, P. E. (1985). College students' perceptions of the advantages and disadvantages of obtaining psychotherapy. Journal of Social Service Research, 8, 75-86. doi:10.1300/J079v08n04_05

Hanson, W. E., Creswell, J. W., Plano Clark, V. L., Petska, K. S., Creswell, D. J. (2005). Mixed methods research designs in counseling psychology. Journal of Counseling Psychology, 52, 224-235. doi: 10.1037/0022-0167.52.2.224

Haverkamp, B. E., Morrow, S. L., \& Ponterotto, J. G. (2005). A time and place for qualitative and mixed methods in counseling psychology research. Journal of Counseling Psychology, 52, 123-125. doi: 10.1037/0022-0167.52.2.123

Heggins, W. J., \& Jackson, J. F. L. (2003). Understanding the collegiate experience for Asian international students at a Midwestern research university. College Student Journal, 37, 379-391.

Ho, D. (1991). The concept of "face" in Chinese-American interaction. In W.C. Hu \& C. L. Grove (Eds.), Encountering the Chinese: A guide for Americans (pp. 111-124). Yarmouth, ME: Intercultural Press.

Ho, D. Y. F. (1982). Asian concepts in behavioral science. Psychologia: An International Journal of Psychology in the Orient, 25, 228-235.

Holmbeck, G. M. (1997). Toward terminological, conceptual, and statistical clarity in the study of mediators and moderators: Examples from the child-clinical and pediatric psychology literatures. Journal of Consulting and Clinical Psychology, 65, 699710. doi:10.1037/0022-006X.65.4.599

Hu, H. C. (1944). The Chinese concept of "face." American Anthropologist, 46, 45-64.

Hu, L. T., \& Bentler, P. M. (1999). Cutoff criteria for fit indexes in covariate structure analysis: Conventional criteria versus new alternatives. Structural Equation Modeling, 6, 1-55.

Huyn, J., Quinn, B., Madon, T., \& Lustig, S. (2007). Mental health need, awareness, and use of counseling services among international graduate students. Journal of American College Health, 56, 109-118. doi:10.3200/JACH.56.2.109-118 
Ibrahim, F. A., \& Ingram, M. A. (2007). Counseling South Asian international students. In H. Singaravelu \& M. Pope (Eds.) A handbook for counseling international students in the United States (pp. 195-211). Alexandria, VA: American Counseling Association.

Institute for International Education. (2010). Open doors 2009: Report on international educational exchange. Retrieved from http://opendoors.iienetwork.org/?p=150808

Johnson, L.R., \& Sandhu, D.S. (2007). Isolation, adjustment, and acculturation issues of international students: Intervention strategies for counselors. In H. Singaravelu \& M. Pope (Eds.) A handbook for counseling international students in the United States (pp. 13-37). Alexandria, VA: American Counseling Association.

Jung, E., Hecht, M. L., \& Wadsworth, B. C. (2007). The role of identity in international students' psychological well-being in the United States: A model of depression level, identity gaps, discrimination, and acculturation. International Journal of Intercultural Relations, 31, 605-624. doi:10.1016/j.ijintrel.2007.04.001

Kaczmarek, P. G., Matlock, G., Merta, R., Ames, M. H., \& Ross, M. (1995). An assessment of international college student adjustment. International Journal for the Advancement of Counseling, 17, 241-247. doi:10.1007/BF01407740

Karr, C. A., \& Larson, L. M. (2005). Use of theory-driven research in counseling: Investigating three counseling psychology journals from 1990 to 1999. The Counseling Psychologist, 33, 299-326. doi:10.1177/0011000004272257

Khoo, P., \& Abu-Rasain, M. (1994). Counseling foreign students: A review of strategies. Counseling Psychology Quarterly, 7, 117-127. doi:10.1080/09515079408254140

Kim, B. S. K. (2007). Adherence to Asian and European American cultural values and attitudes toward seeking professional psychological help among Asian American college students. Journal of Counseling Psychology, 54, 474-480. doi:10.1037/0022-0167.54.4.474

Kim, B. S. K., \& Abreu, J. M. (2001). Acculturation measurement: Theory, current instruments, and future directions. In J. G. Ponterotto, J. M. Casas, L. A. Suziki, \& C. M. Alexander (Eds.), Handbook of multicultural counseling $\left(2^{\text {nd }}\right.$ ed., pp. 394-424). Thousand Oaks, CA: Sage.

Kim, B. S. K., Atkinson, D. R., \& Yang, P. H. (1999). The Asian Values Scale: Development, factor analysis, validation, and reliability. Journal of Counseling Psychology, 46, 342-352. doi:10.1037/0022-0167.46.3.342 
Kim, B. S. K., Li, L. C., \& Ng, G. F. (2005). Asian American Values Scale Multidimensional: Development, reliability, and validity. Cultural Diversity and Ethnic Minority Psychology, 11, 187-201. doi:10.1037/1099-9809.11.3.187

Kim, B. S. K., \& Omizo, M. M. (2003). Asian cultural values, attitudes toward seeking professional psychological help, and willingness to see a counselor. The Counseling Psychologist, 31, 343-361. doi:10.1177/0011000003252268

Kim, S., \& Hunter, J. E. (1993). Relationships among attitudes, behavioral intentions, and behavior: A meta-analysis of past research, part 2. Communication Research, 20, 331-364. doi:10.1177/009365093020003001

Kline, R. B. (2005). Principles and practice of structural equation modeling. New York, NY: Guilford Press.

Komiya, N., \& Eells, G. T. (2001). Predictors of attitudes toward seeking counseling among international students. Journal of College Counseling, 4, 153-160.

Komiya, N., Good, G. E., \& Sherrod, N. B. (2000). Emotional openness as a predictor of college students' attitudes toward seeking psychological help. Journal of Counseling Psychology, 47, 138-143. doi:1037/0022-0167.47.1.138

Lacina, J. G. (2002). Preparing international students for a successful social experience in higher education. New Directions for Higher Education, 117, 21-27.

Lent, R. W. Brown, S. D., \& Hackett, G. (1996). Career development from a social cognitive perspective. In D. Brown and L. Brooks (Eds.), Career choice and development (pp. 372 - 421). San-Francisco, CA: Jossey Bass, Inc.

Leung, A. S. (2007). Returning home and issues related to reverse culture shock. In H. Singaravelu \& M. Pope (Eds.) A handbook for counseling international students in the United States (pp. 137-154). Alexandria, VA: American Counseling Association.

Leong, F. T. L., \& Chou, E. L. (1996). Counseling international students. The Counseling Psychologist, 24, 210-242. doi:10.1177/0011000091191002

Leong, F. T. L., Wagner, N., \& Kim, H. H. (1995). Group counseling expectations among Asian American students: The role of culture-specific factors. Journal of Counseling Psychology, 47, 217-222. doi:10.1037/0022-0167.42.2.217

Liao, H., Rounds, J., \& Klein, A. G. (2005). A test of Cramer's (1999) help-seeking model and acculturation effects with Asian and Asian American college students. Journal of Counseling Psychology, 52, 400-411. doi:10.1037/0022-0167.52.3.400 
Lin, S. \& Pedersen, P. B. (2007). Multinational competencies of international student service providers. In H. Singaravelu \& M. Pope (Eds.) A handbook for counseling international students in the United States (pp. 285-299). Alexandria, VA: American Counseling Association.

Lin, J. G., \& Yi, J. K. (1997). Asian international students' adjustment: Issues and program suggestions. College Student Journal, 31, 473-479.

Lippincott, J. A., \& Mierzwa, J. A. (1995). Propensity for seeking counseling services: A comparison of Asian and American undergraduates. Journal of American College Health, 43, 201-205.

Mau, W., \& Jepsen, D. A. (1990). Help-seeking perceptions and behaviors: A coparison of Chinese and American graduate students. Journal of Multicultural Counseling and Development, 67, 189-192.

Miller, M. J. (2007). A bilinear multidimensional model of Asian American acculturation and enculturation: Implications for counseling interventions. Journal of Counseling Psychology, 54, 118-131. doi:10.1037/0022-0167.54.2.118

Mitchell, S. L., Greenwood, A. K., \& Guglielmi, M. C. (2007).Utilization of counseling services: Comparing international and U.S. college students. Journal of College Counseling, 10, 117-129.

Miville, M. L., \& Constantine, M. G. (2007). Cultural values, counseling stigma, and intentions to seek counseling among Asian American college women. Counseling and Values, 2-11.

Misra, R., Crist, M., \& Burant, C. J. (2003). Relationships among life stress, social support, academic stressors, and reactions to stressors of international students in the United States. International Journal of Stress Management, 10, 137-157. doi: $10.1037 / 1072-5245.10 .2 .137$

Mo, P. K. H., \& Mak, W. S. (2009). Help-seeking for mental health problems among Chinese: The application and extension of the theory of planned behavior. Social Psychiatry and Psychiatric Epidemiology, 44, 675-684. doi:10.1007/s00127-0080484-0

Mori, S. (2000). Addressing the mental health concerns of international students. Journal of Counseling and Development, 78, 137-145.

Morrow, S. L. (2007). Qualitative research in counseling psychology: Conceptual Foundations. The Counseling Psychologist, 35, 209-235. doi: $10.1177 / 0011000006286990$ 
Murdock, N. L. (2006). On science-practice integration in everyday life: A plea for theory. The Counseling Psychologist, 34, 548-569.

doi: $10.1177 / 0011000006288129$

Nilsson, J. E., Berkel, L. A., Flores, L. Y., \& Lucas, M. S. (2004). Utilization rate and presenting concern of international students at a university counseling center: Implications for outreach programming. Journal of College Student Psychotherapy, 19, 49-59. doi:10.1300/J035v19n02_05

Olivas, M., \& Li, C. (2006). Understanding stressors for international students in higher education: What college counselors and personnel need to know. Journal of Instructional Psychology, 33, 217-222.

Pedersen, P. B. (1991). Counseling international students. The Counseling Psychologist, 19, 10-58. doi:10.1177/0011000091191002

Prieto, S. L. (1995). International student populations and needs assessment. In S. D. Stabb, S. M. Harris, \& J. E., Talley (Eds.) Multicultural needs assessment for college and university student populations (pp. 203-223). Springfield, IL: Charles C Thomas.

Poyrazli, S., Kavanaugh, P. R., \& Baker, A. (2004). Social support and demographic correlates of acculturative stress in international students. Journal of College Counseling, 7, 73-82.

Romano, J. L., \& Netland, J. D. (2008). The application of the Theory of Reasoned Action and Planned Behavior to prevention science in counseling psychology. The Counseling Psychologist, 36, 777-806. doi: 10.1177/0011000007301670

Satorra, A. \& Bentler, P. M. (2001). A scaled difference chi-square test statistic for moment structure analysis. Psychometrika, 66, 507-514. doi:10.1007/BF02296192

Schomerus, G., \& Angermeyer, M. C. (2008). Stigma and its impact on help-seeking for mental disorders: What do we know? Epidemiologia e Psychiatri Sociale, 17, 3137.

Shea, M., \& Yeh, C. J. (2008). Asian American students' cultural values, stigma, and relational self-construal: Correlates of attitudes toward professional help seeking. Journal of Mental Health Counseling, 30, 157-172.

Sheu, H., \& Fukuyama, M. A. (2007). Counseling international students from East Asia. In H. Singaravelu \& M. Pope (Eds.) A handbook for counseling international students in the United States (pp. 173-195). Alexandria, VA: American Counseling Association. 
Shrout, P. E., \& Bolger, N. (2002). Mediation in experimental and non-experimental studies: New procedures and recommendations. Psychological Methods, 7, 422445. doi:10.1037/1082-989X.7.4.422

Sodowsky, G. R., \& Plake, B. S. (1991). Psychometric properties of the AmericanInternational Relations Scale. Educational and Psychological Measurement, 51, 207-216. doi: 10.1177/0013164491511020

Sodowsky, G. R., \& Plake, B. S. (1992). A study of acculturation differences among international people and suggestions for sensitivity to within-group differences. Journal of Counseling and Development, 71, 53-59.

Sue, D. W., \& Sue, D. (2008). Counseling the culturally diverse: Theory and practice $\left(5^{\text {th }}\right.$ ed.). New York, NY: Wiley \& Sons, Inc.

Suinn, R. M., Ahuna, C., \& Khoo, G. (1992). The Suinn-Lew Asian Self-Identity Acculturation Scale: Concurrent and factor validation. Educational and Psychological Measurement, 52, 1041-1046. doi:10.1177/0013164492052004028

Suinn, R. M., \& Khoo, G. (1995). The Suinn-Lew Asian Self-Identity Acculturation Scale: Cross-cultural information. Journal of Multicultural Counseling and Development, 23, 139-150.

Suinn, R. M., Rickard-Figueroa, K., Lew, S., \& Vigil, P. (1987). The Suinn-Lew Asian Self-Identity Acculturation Scale: An initial report. Educational and Psychological Measurement, 47, 401-407. doi:10.1177/0013164487472012

Surdam, J. C., \& Collins, J. R. (1984). Adaptation of international students: A cause for concern. Journal of College Student Personnel, 25, 240-245.

Swagler, M. A., \& Ellis, M. V. (2003). Crossing the distance: Adjustment of Taiwanese graduate students in the United States. Journal of Counseling Psychology, 50, 420-437. doi:10.1037/0022-0167.50.4.420

Tabachnick, B. G., \& Fidell, L. S. (2007). Using multivariate statistics (5th Ed.). New York: Harper Collins.

Tata, S. P., \& Leong, F. T. L. (1994). Individualism-collectivism, social network orientation, and acculturation as predictors of attitudes toward seeking professional psychological help among Chinese Americans. Journal of Counseling Psychology, 41, 280-287. doi:10.1037/0022-0167.41.3.280 
Tedeschi, G. J., \& Willis, F. N. (1993). Attitudes toward counseling among Asian international and native Caucasian students. Journal of College Student Psychotherapy, 7, 43-54. doi:10.1300/J035v07n04_04

Tidwell, R., \& Hanassab, S. (2007). New challenges for professional counselors: The higher education international student population. Counseling Psychology Quarterly, 20, 313-324. doi:10.1080/09515070701573927

Ting, J. Y., \& Hwang, W. (2009). Cultural influences on help-seeking attitudes in Asian American students. American Journal of Orthopsychiatry, 79, 125-132. doi:10.1037/a0015394

Toyokawa, T., \& Toyakawa, N. (2002). Extracurricular activities and the adjustment of Asian international students: A study of Japanese students. International Journal of Intercultural Relations, 26, 363-379. doi:10.1016/S0147-1767(02)00010-X

United States Census Bureau. (2007). The American community - Asians: 2004. American Community Survey Reports. Retrieved from: https://www.census.gov/prod/2007pubs/acs-05.pdf

Vogel, D. L., Wade, N. G., \& Ascheman, P. L. (2009). Measuring perceptions of stigmatization by others for seeking psychological help: Reliability and validity of a new stigma scale with college students. Journal of Counseling Psychology, 56, 301-308. doi:10.1037/a0014903

Vogel, D. L., Wade, N. G., \& Haake, S. (2006). Measuring the self-stigma associated with seeking psychological help. Journal of Counseling Psychology, 53, 325-337. doi:10.1037/0022-0167.53.3.325

Vogel, D. L., Wester, S. R., \& Larson, L. M. (2007). Avoidance of counseling: Psychological factors that inhibit seeking help. Journal of Counseling and Development, 85 , 410-422.

Vogel, D. L., Wester, S. R., Wei, M., \& Boysen, G. A. (2005). The role of outcome expectations and attitudes on decisions to seek professional help. Journal of Counseling Psychology, 52, 459-470. doi:10.1037/0022-0167.52.4.459

Wadsworth, B. C., Hecht, M. L., \& Jung, E. (2008). The role of identity gaps, discrimination, and acculturation in international students' satisfaction in American classrooms. Communication Education, 57, 64-87. doi: $10.1080 / 03634520701668407$ 
Wang, Y., Lin, J. G., Pang, L., \& Shen, F. C. (2007). International students from Asia. In F. T. Leong, A. Ebreo, L. Kinoshita, A. G. Inman, L. H. Yang, \& M. Fu (Eds.) Handbook of Asian American Psychology (2 ${ }^{\text {nd }}$ ed., pp 245-261). Thousand Oaks, CA: Sage Publications.

Weston, R., \& Gore, P. A. (2006). A brief guide to structural equation modeling. The Counseling Psychologist, 34, 719-751. doi:10.1177/0011000006286345

Wei, M., Heppner, P.P., Mallen, M. J., Ku, T., Liao, K., \& Wu, T. (2007). Acculturative stress, perfectionism, years in the United States, and depression among Chinese international students. Journal of Counseling Psychology, 54, 385-394. doi:10.1037/0022-0167.54.4.385

Wilson, C. J., Deane, F. P., Ciarrochi, J., \& Rockwood, D. (2005). Measuring helpseeking intentions: Properties of the General Help Seeking Questionnaire. Canadian Journal of Counseling, 39, 15-28.

Wilton, L. \& Constantine, M. G. (2003). Length of residence, cultural adjustment difficulties, and psychological distress symptoms in Asian and Latin American international college students. Journal of College Counseling, 6, 177-186.

Wolfe, M. M., Yang, P. H., Wong, E. C., \& Atkinson, D. R. (2001). Design and development of the European American Values Scale for Asian Americans. Cultural Diversity and Ethnic Minority Psychology, 7, 274-283. doi:10.1037/1099-9809.7.3.274

Yakushko, O., Davidson, M. M., Sanford-Martens, T. C. (2008). Seeking help in a foreign land: International students' use patterns for a U.S. university counseling center. Journal of College Counseling, 11, 6-18.

Yeh, C. J., \& Inose, M. (2003). International students' reported English fluency, social support satisfaction, and social connectedness as predictors of acculturative stress. Counseling Psychology Quarterly, 16, 15-28. doi:10.1080/0951507031000114058

Yi, J. K., Lin, J. G., \& Kishimoto, Y. (2003). Utilization of counseling services by international students. Journal of Instructional Psychology, 30, 333-342.

Ying, Y. (2005). Variation in acculturative stress over time: A study of Taiwanese students in the United States. International Journal of Intercultural Relations, 29, 59-71. doi:10.1016/j.ijintrel.2005.04.003

Ying, Y. W., \& Liese, L. H. (1994). Initial adjustment of Taiwanese students to the United States: The impact of postarrival variables. Journal of Cross-Cultural Psychology, 25, 466-477. doi: 10.1177/0022022194254003 
Yong, Y. M. A. (2007). Chinese and Chinese American parents' intentions to seek psychological services for their children: The applicability of the theory of reasoned action. Dissertation Abstracts International: Section B: The Sciences and Engineering, 68, 1326.

Yoo, S., Goh, M. \& Yoon, E. (2005). Psychological and cultural influences on Korean's help-seeking attitudes. Journal of Mental Health Counseling, 27, 266-281.

Yoon, E., \& Jepsen, D. A. (2008). Expectations of and attitudes toward counseling: A comparison of Asian international and U.S. graduate students. International Journal of the Advancement of Counseling, 30, 116-127. doi:10.1007/s10447008-9050-4

Yoon, E., \& Portman, T. A. A. (2004). Critical issues of literature on counseling international students. Journal of Multicultural Counseling and Development, 32, $33-44$.

Zane, N. (1991). An empirical examination of loss of face among Asian Americans. Unpublished manuscript, Graduate School of Education, University of California, Santa Barbara.

Zane, N., \& Mak, W. (2003). Major approaches to the measurement of acculturation among ethnic minority populations: A content analysis and an alternative empirical strategy. In K. M. Chun, P. B. Organista, and G. Marin (Eds.), Acculturation: Advances in theory, measurement, and applied research (pp. 123139). New York: Kluwer Academic/Plenum Press.

Zane, N., \& Yeh, M. (2002). The use of culturally-based variables in assessment: Studies on loss of face. In K. Kurasaki, S. Okazaki, \& S. Sue (Eds.), Asian American mental health: Assessment theories and methods (pp. 123-138). New York, NY: Kluwer Academic/Plenum Publishers.

Zayco, R. A. (2009). Asian American cultural values, loss of face, and self-concealment as predictors of attitudes toward seeking professional psychological help. Dissertation Abstract International: Section B: The Sciences and Engineering, 69, 4451.

Zhang, N., \& Dixon, D. N. (2001). Multiculturally responsive counseling: Effects on Asian students' ratings of counselors. Journal of Multicultural Counseling and Development, 29, 253-262.

Zhang, N., \& Dixon, D. N. (2003). Acculturation and attitudes of Asian international students toward seeking psychological help. Journal of Multicultural Counseling and Development, 31, 205-222. 
APPENDICES 


\section{APPENDIX A}

\section{IRB LETTER OF APPROVAL}

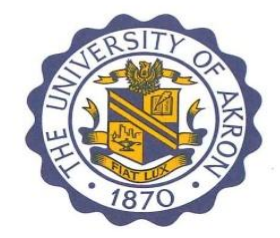

NOTICE OF APPROVAL

June 2, 2010

Elena Yakunina

177 Olive Street

Akron, Ohio 44310

From: Sharon McWhorter, IRB Administrator

Re: IRB Number 20100513 “" Asian International Students' Intention to Seek Counseling: Integrating Cognitive and Cultural Predictors"

Thank you for submitting your Exemption Request for the referenced study. Your request was approved on June 2, 2010. The protocol represents minimal risk to subjects and matches the following federal category for exemption:

$\square$ Exemption 1 - Research conducted in established or commonly accepted educational settings, involving normal educational practices.

$\bigotimes$ Exemption 2 - Research involving the use of educational tests, survey procedures, interview procedures, or observation of public behavior.

Exemption 3 - Research involving the use of educational tests, survey procedures, interview procedures, or observation of public behavior not exempt under category 2 , but subjects are elected or appointed public officials or candidates for public office.

$\square$ Exemption 4 - Research involving the collection or study of existing data, documents, records, pathological specimens, or diagnostic specimens.

Exemption 5 - Research and demonstration projects conducted by or subject to the approval of department or agency heads, and which are designed to study, evaluate, or otherwise examine public programs or benefits.

Exemption 6 - Taste and food quality evaluation and consumer acceptance studies.

Annual continuation applications are not required for exempt projects. If you make changes to the study's design or procedures that increase the risk to subjects or include activities that do not fall within the approved exemption category, please contact me to discuss whether or not a new application must be submitted. Any such changes or modifications must be reviewed and approved by the IRB prior to implementation.

Please retain this letter for your files. If the research is being conducted for a master's thesis or doctoral dissertation, the student must file a copy of this letter with the thesis or dissertation.

Cc: Ingrid Weigold - Advisor

$\bigotimes$ Approved consent form/s enclosed

Cc: Stephanie Woods - IRB Chair

$$
\begin{aligned}
& \text { Office of Research Services and Sponsored Programs } \\
& \text { Akron, } \mathrm{OH} 44325-2102 \\
& 330-972-7666 \cdot 330-972-6281 \text { Fax } \\
& \text { The University of Akron is an Equal Education and Employment Institution }
\end{aligned}
$$




\section{APPENDIX B}

\section{LEADING INSTITUTIONS HOSTING INTERNATIONAL STUDENTS}

\begin{tabular}{|c|c|c|c|}
\hline Rank & Institution & City, State & $\begin{array}{l}\text { Total International } \\
\text { Students }\end{array}$ \\
\hline 1 & University of Southern California & Los Angeles, CA & 7,482 \\
\hline 2 & New York University & New York, NY & 6,761 \\
\hline 3 & Columbia University & New York, NY & 6,685 \\
\hline 4 & University of Illinois Urbana-Champaign & Champaign, IL & 6,570 \\
\hline 5 & Purdue University - Main Campus & West Lafayette, IN & 6,136 \\
\hline 6 & University of Michigan - Ann Arbor & Ann Arbor, MI & 5,790 \\
\hline 7 & University of Texas - Austin & Austin, TX & 5,703 \\
\hline 8 & University of California - Los Angeles & Los Angeles, CA & 5,590 \\
\hline 9 & Boston University & Boston, MA & 5,037 \\
\hline 10 & Michigan State University & East Lansing, MI & 4,757 \\
\hline 11 & Harvard University & Cambridge, MA & 4,735 \\
\hline 12 & University of Florida & Gainesville, FL & 4,731 \\
\hline 13 & University of Pennsylvania & Philadelphia, PA & 4,635 \\
\hline 14 & Ohio State University - Main Campus & Columbus, $\mathrm{OH}$ & 4,583 \\
\hline 15 & Indiana University - Bloomington & Bloomington, IN & 4,565 \\
\hline 16 & Texas A\&M University & College Station, TX & 4,558 \\
\hline 17 & SUNY University at Buffalo & Buffalo, NY & 4,539 \\
\hline 18 & Arizona State University - Tempe Campus & Tempe, AZ & 4,375 \\
\hline 19 & University of Wisconsin - Madison & Madison, WI & 4,243 \\
\hline 20 & University of Minnesota - Twin Cities & Minneapolis, MN & 4,120 \\
\hline 21 & Cornell University & Ithaca, NY & 4,018 \\
\hline 22 & Stanford University & Stanford, CA & 3,976 \\
\hline
\end{tabular}




\begin{tabular}{|c|c|c|c|}
\hline 23 & Georgia Institute of Technology & Atlanta, GA & 3,911 \\
\hline 24 & University of Houston & Houston, TX & 3,778 \\
\hline 25 & Penn State University - University Park & University Park, PA & 3,741 \\
\hline 26 & University of California - Berkeley & Berkeley, CA & 3,506 \\
\hline 27 & University of Maryland - College Park & College Park, MD & 3,480 \\
\hline 28 & Massachusetts Institute of Technology & Cambridge, MA & 3,408 \\
\hline 29 & University of Washington & Seattle, WA & 3,382 \\
\hline 30 & Illinois Institute of Technology & Chicago, IL & 3,342 \\
\hline 31 & Carnegie Mellon University & Pittsburgh, PA & 3,299 \\
\hline 32 & Northeastern University & Boston, MA & 3,242 \\
\hline 33 & University of Texas - Arlington & Arlington, TX & 3,182 \\
\hline 34 & Santa Monica College & Santa Monica, CA & 3,147 \\
\hline 35 & Florida International University & Miami, FL & 2,954 \\
\hline 36 & University of Arizona & Tucson, AZ & 2,913 \\
\hline 37 & North Carolina State University & Raleigh. NC & 2,879 \\
\hline 38 & Academy of Art University & San Francisco, CA & 2,835 \\
\hline 39 & University of Chicago & Chicago, IL & 2,789 \\
\hline 40 & SUNY Stony Brook University & Stony Brook, NY & 2,772 \\
\hline 41 & University of Texas - Dallas & Richardson, TX & 2,770 \\
\hline 42 & Iowa State University & Ames, IA & 2,734 \\
\hline 43 & Wayne State University & Detroit, MI & 2,632 \\
\hline 44 & DeAnza College & Cupertino, CA & 2,595 \\
\hline 45 & University of Bridgeport & Bridgeport, CT & 2,575 \\
\hline 46 & University of Illinois Chicago & Chicago, IL & 2,563 \\
\hline 47 & John Hopkins University & Baltimore, MD & 2,562 \\
\hline 48 & Rutgers University & New Brunswick, NJ & 2,549 \\
\hline 49 & San Jose State University & San Jose, CA & 2,522 \\
\hline 50 & Virginia Polytechnic Institute & Blacksburg, VA & 2,522 \\
\hline 51 & California State University - Long Beach & Long Beach, CA & 2,508 \\
\hline
\end{tabular}




\begin{tabular}{|c|c|c|c|}
\hline 52 & Duke University & Durham, NC & 2,466 \\
\hline 53 & San Francisco State University & San Francisco, CA & 2,451 \\
\hline 54 & University of Central Florida & Orlando, FL & 2,446 \\
\hline 55 & University of North Texas & Denton, TX & 2,426 \\
\hline 56 & George Washington University & Washington, DC & 2,406 \\
\hline 57 & University of Iowa & Iowa City, IA & 2,379 \\
\hline 58 & Northwestern University & Evanston, IL & 2,357 \\
\hline 59 & SUNY Binghamton & Binghamton, NY & 2,285 \\
\hline 60 & Montgomery College & Rockville, MD & 2,248 \\
\hline 61 & California State University - Northridge & Northridge, CA & 2,222 \\
\hline 62 & University of California - Davis & Davis, CA & 2,221 \\
\hline 63 & University of Pittsburgh & Pittsburgh, PA & 2,216 \\
\hline 64 & The New School & New York, NY & 2,209 \\
\hline 65 & Lone Star College & The Woodlands, TX & 2,174 \\
\hline 66 & George Manson University & Fairfax, VA & 2,158 \\
\hline 67 & University of Cincinnati & Cincinnati, OH & 2,157 \\
\hline 68 & Bingham Young University & Provo, UT & 2,142 \\
\hline 69 & Yale University & New Haven, CT & 2,094 \\
\hline 70 & University of Texas - El Paso & El Paso, TX & 2,078 \\
\hline 71 & University of California - San Diego & La Jolla, CA & 2,010 \\
\hline 72 & Drexel University & Philadelphia, PA & 2,007 \\
\hline 73 & Wichita State University & Wichita, KS & 1,939 \\
\hline 74 & University of Virginia - Main Campus & Charlottesville, VA & 1,929 \\
\hline 75 & Temple University & Philadelphia, PA & 1,919 \\
\hline
\end{tabular}




\section{APPENDIX C \\ PARTICIPANT E-MAIL INVITATION}

Dear Fellow International Student,

My name is Elena Yakunina, and I am an international student from Bulgaria and a doctoral candidate in Counseling Psychology. I would like to invite you to participate in my dissertation research, which examines Asian international students' attitudes toward seeking counseling. To be eligible to participate, you need to be an Asian international student holding a valid U.S. student visa. International students coming from the following regions are eligible: East Asia, Southeast Asia, and South and Central Asia.

If you choose to participate, you will complete a 15 - to 20 -minute online survey. At the end, you will have the option to provide your name and e-mail address to win one of five \$200 American Express gift cards. The information you provide will be kept confidential and your name will not be associated with your responses to the survey.

If you would like to participate in this study, please click on the web link provided below. It will direct you to the online survey developed for the purpose of this study.

If you have any questions, please do not hesitate to contact the primary researcher, Elena Yakunina, at esy1@zips.uakron.edu.

Thank you in advance for your time! 


\section{APPENDIX D \\ PARTICIPANT INFORMED CONSENT FORM}

Dear Fellow International Student,

You are invited to participate in a research study being conducted by Elena Yakunina, a doctoral candidate in Counseling Psychology at The University of Akron, under the direction of Dr. Ingrid Weigold.

Title of Study: Asian International Students' Intentions to Seek Counseling: Integrating Cognitive and Cultural Predictors

Purpose: The purpose of this study is to explore Asian international students' cultural beliefs and attitudes toward seeking counseling.

Eligibility: To be eligible to participate, you need to be an Asian international student holding a valid U.S. student visa. International students coming from the following geographic regions are eligible: East Asia, Southeast Asia, and South and Central Asia.

Procedures: If you choose to participate, you will complete an on-line survey that will take between 15 and 20 minutes of your time.

Risks and Discomforts: Participation in this study is not believed to be associated with any significant risks or discomfort.

Benefits: You will receive no direct benefits from participation. However, your participation may help researchers understand Asian international students' cultural beliefs and attitudes toward seeking counseling.

Incentive to Participants: At the end of the survey, you will have the option to provide your name and email address to win one of five \$200 American Express gift cards.

Right to Refuse or Withdraw: Taking part in this study is voluntary. If you decide that you do not want to participate, you can withdraw from the study at any time.

Anonymous and Confidential Data Collection: The information you provide will be kept anonymous and your name will not be associated with your responses to the survey. Any papers resulting from this study will report data in an aggregate manner. 
Who to Contact with Questions: If you have any questions about this research, do not hesitate to email Elena Yakunina at esy1@zips.uakron.edu or to contact her advisor, Dr. Ingrid Weigold, at weigold@uakron.edu. This research project has been reviewed and approved by The University of Akron Institutional Review Board for the Protection of Human Subjects. Questions about your rights as a research participant can be directed to Sharon McWhorter, Associate Director of Research Services, at 1-330-972-7666.

Acceptance and Consent: I have read the information provided and all of my questions have been answered. I voluntarily agree to participate in this study. My completion of this survey will serve as my consent. I may print a copy of this consent statement for future reference.

a) Yes, I consent to participate in this study.

b) No, I decline to participate and wish to exit this study. 


\section{APPENDIX E \\ OPTIONAL RAFFLE ENTRY FORM}

\section{Dear Fellow International Student,}

Thank you for taking participation in this study! Please complete the entry form below if you would like to be included in a raffle for one of five \$200 American Express gift cards. This will allow us to contact you in the event that you are selected for this prize. The information you provide will be stored separately from your survey responses in order to preserve your anonymity.

If you would like to be included in this raffle, please provide the following information:

First Name:

Last Name:

Email Address:

Thank you again for your participation! 


\section{APPENDIX F}

\section{DEMOGRAPHIC QUESTIONNAIRE}

1. Please list your age:

2. Please select your sex:

a) Man

b) Woman

3. Select your relationship status from the options below:
a) Single
b) Partnered
c) Married
d) Separated
e) Divorced
f) Widowed
g) Other (Please specify

4. Pease select your ethnic/racial background from the options below:
a. Asian
b. European
c. African
d. American Indian
e. Hispanic / Latino/a
f. Biracial / Multiracial (Please specify ___ _
g. Other (Please specify

5. Please list your country of origin:

6. Please select your U.S. Visa Type from the options below:
a) $\mathrm{J}-1$
b) F-1
c) $\mathrm{M}-1$
d) K-1
e) U.S. resident
f) U.S. citizen
g) Other (Please specify

7. Please list the number of years you have lived in the United States: 
8. Please select your academic status from the options below:
a) Freshman
b) Sophomore
c) Junior
d) Senior
e) Graduate student
f) Non-degree student
g) Other (Please specify

9. Please select your field of study from the options below:
a) Business and management
b) Engineering
c) Physical and life sciences
d) Social sciences
e) Math and computer science
f) Fine and applied arts
g) Health care professions
h) Education
i) Intensive English study
j) Humanities
k) Agriculture
1) Other (Please specify

10. Please list your academic institution:

11. Have you ever seen a mental health professional (e.g., school counselor, counselor, social worker, psychologist, psychiatrist) to get help for a personal or an emotional problem?
a) Yes
b) No (Skip to Question 13)

12. If you responded "Yes" to question 10, where did you seek professional mental health services?
a) In your home country
b) In the United States
c) Both in your home country and in the United States
d) Other (Please specify

13. If you responded "Yes" to question 10, what kind of mental health professional did you see?
a) School counselor
b) Counselor
c) Social worker
d) Psychologist 
e) Psychiatrist

f) (Please specify

14. Are you aware of any on-campus mental health resources that offer help for personal and emotional problems?

a) Yes

b) No

If yes, please list:

15. Are you aware of any off-campus mental health resources that offer help for personal and emotional problems?

a) Yes

b) No

If yes, please list: 


\section{APPENDIX G}

\section{INTETIONS TO SEEK COUNSELING INVENTORY - PSYCHOLOGICAL AND INTERPERSONAL CONCERNS}

Directions: Please respond to the following items as accurately and honestly as possible. There are no wrong answers to these questions. It is important that you answer every item. Please select the number for each statement to indicate your response.

\begin{tabular}{|c|c|c|c|c|}
\hline $\begin{array}{l}\text { Below is a list of issues people commonly bring to counseling. } \\
\text { How likely would you be to seek counseling if you were } \\
\text { experiencing these problems? }\end{array}$ & $\begin{array}{l}\frac{\lambda}{0} \\
\frac{y}{=} \\
\bar{J} \\
\overrightarrow{0} \\
\overrightarrow{0}\end{array}$ & $\frac{2}{\stackrel{2}{a}}$ & $\begin{array}{l}\frac{\lambda}{2} \\
\frac{0}{3}\end{array}$ & $\begin{array}{l}\frac{\lambda}{0} \\
\frac{0}{3} \\
\frac{2}{0} \\
>\end{array}$ \\
\hline 1. Relationship difficulties & 1 & 2 & 3 & 4 \\
\hline 2. Concerns about sexuality & 1 & 2 & 3 & 4 \\
\hline 3. Depression & 1 & 2 & 3 & 4 \\
\hline 4. Conflict with parents & 1 & 2 & 3 & 4 \\
\hline 5. Difficulties dating & 1 & 2 & 3 & 4 \\
\hline 6. Difficulty sleeping & 1 & 2 & 3 & 4 \\
\hline 7. Inferiority feelings & 1 & 2 & 3 & 4 \\
\hline 8. Difficulties with friends & 1 & 2 & 3 & 4 \\
\hline 9. Self-understanding & 1 & 2 & 3 & 4 \\
\hline 10. Loneliness & 1 & 2 & 3 & 4 \\
\hline
\end{tabular}




\section{APPENDIX H \\ ATTITUDES TOWARD SEEKING \\ PROFESSIONAL PSYCHOLOGICAL HELP SCALE - SHORT FORM}

Directions: Please respond to the following items as accurately and honestly as possible. There are no wrong answers to these questions. It is important that you answer every item. Please select the number for each statement to indicate your response.

Below are a number of statements pertaining to psychology and
mental health issues. Read each statement carefully and
indicate the degree to which you agree or disagree with each
item.


10. Personal and emotional troubles, like many things, tend to work out by themselves. *

Note. Asterisk indicates a reverse-worded item. 


\section{APPENDIX I}

\section{PERCEPTIONS OF STIGMATIZATION BY OTHERS \\ FOR SEEKING PSYCHOLOGICAL HELP SCALE}

Directions: Please respond to the following items as accurately and honestly as possible. There are no wrong answers to these questions. It is important that you answer every item. Please select the number for each statement to indicate your response.

Instructions: Imagine you had a problem that needed to be treated by a mental health professional. If you sought mental health services, to what degree do you believe that the people you interact with would

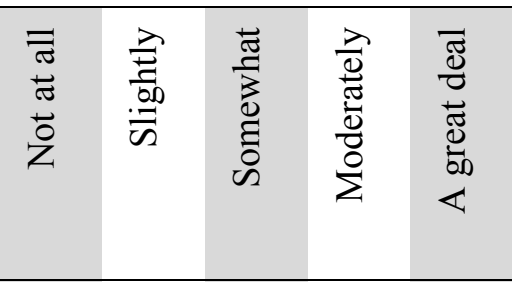

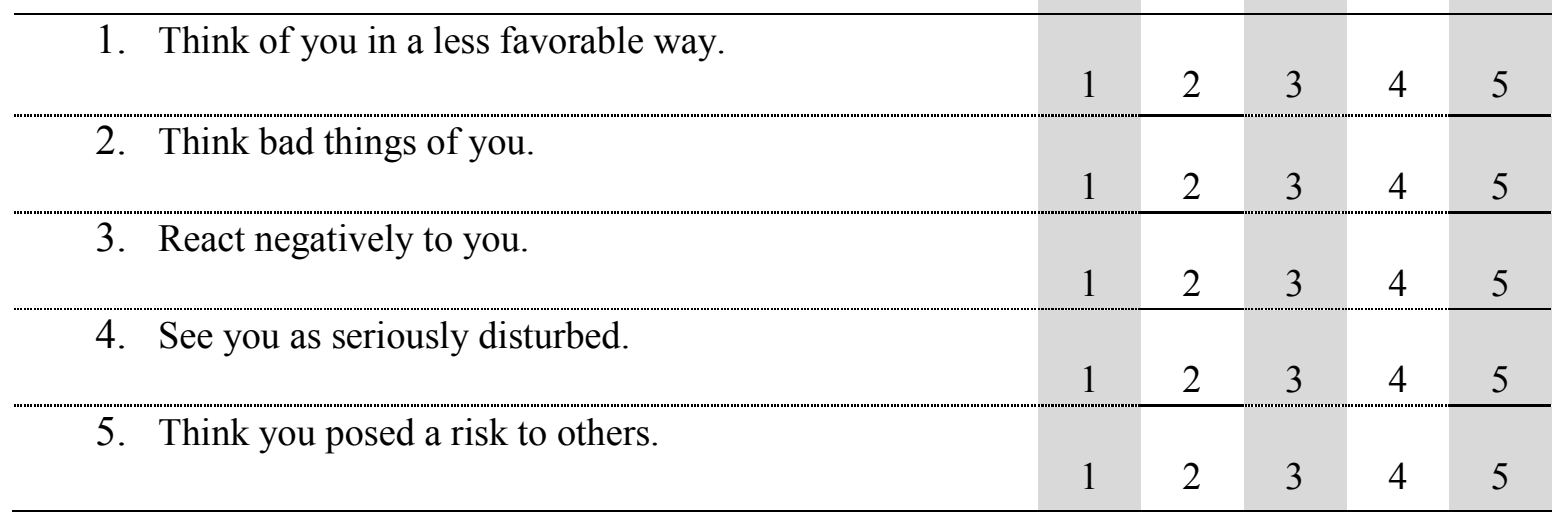




\section{APPENDIX J}

\section{ASIAN AMERICAN MULTIDIMENSIONAL ACCULTURATION SCALE - IDENTIFICATION WITH AMERICAN CULTURE}

Directions: Please respond to the following items as accurately and honestly as possible. There are no wrong answers to these questions. It is important that you answer every item. Please select the number for each statement to indicate your response.

\begin{tabular}{|c|c|c|c|c|c|c|}
\hline & \begin{tabular}{l}
$\bar{z}$ \\
\multirow{2}{*}{} \\
0 \\
$z$
\end{tabular} & 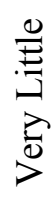 & 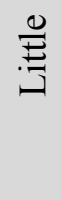 & 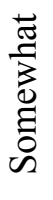 & 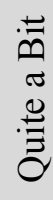 & $\begin{array}{l}\frac{5}{0} \\
\sum_{0}^{0} \\
\overbrace{j}^{0}\end{array}$ \\
\hline 1. How much do you identify with the United States? & & & & & & \\
\hline & 1 & 2 & 3 & 4 & 5 & 6 \\
\hline $\begin{array}{l}\text { 2. How much do you have in common with people in the United } \\
\text { States? }\end{array}$ & 1 & 2 & 3 & 4 & 5 & 6 \\
\hline 3. How proud are you to be a part of the United States? & & & & & & \\
\hline 4 How much do vou interact and associate with neonle from the & & & & & & \\
\hline United States? & 1 & 2 & 3 & 4 & 5 & 6 \\
\hline $\begin{array}{l}\text { 5. How much would you like to interact and associate with } \\
\text { people from the United States? }\end{array}$ & 1 & 2 & 3 & 4 & 5 & 6 \\
\hline $\begin{array}{l}\text { 6. How negative do you feel about people from the United } \\
\text { States? }\end{array}$ & 1 & 2 & 3 & 4 & 5 & 6 \\
\hline 7. How well do you speak American English? & & & & & & \\
\hline & 1 & 2 & 3 & 4 & 5 & 6 \\
\hline 8. How well do you understand American English? & & & & & & \\
\hline & 1 & 2 & 3 & 4 & 5 & 6 \\
\hline
\end{tabular}




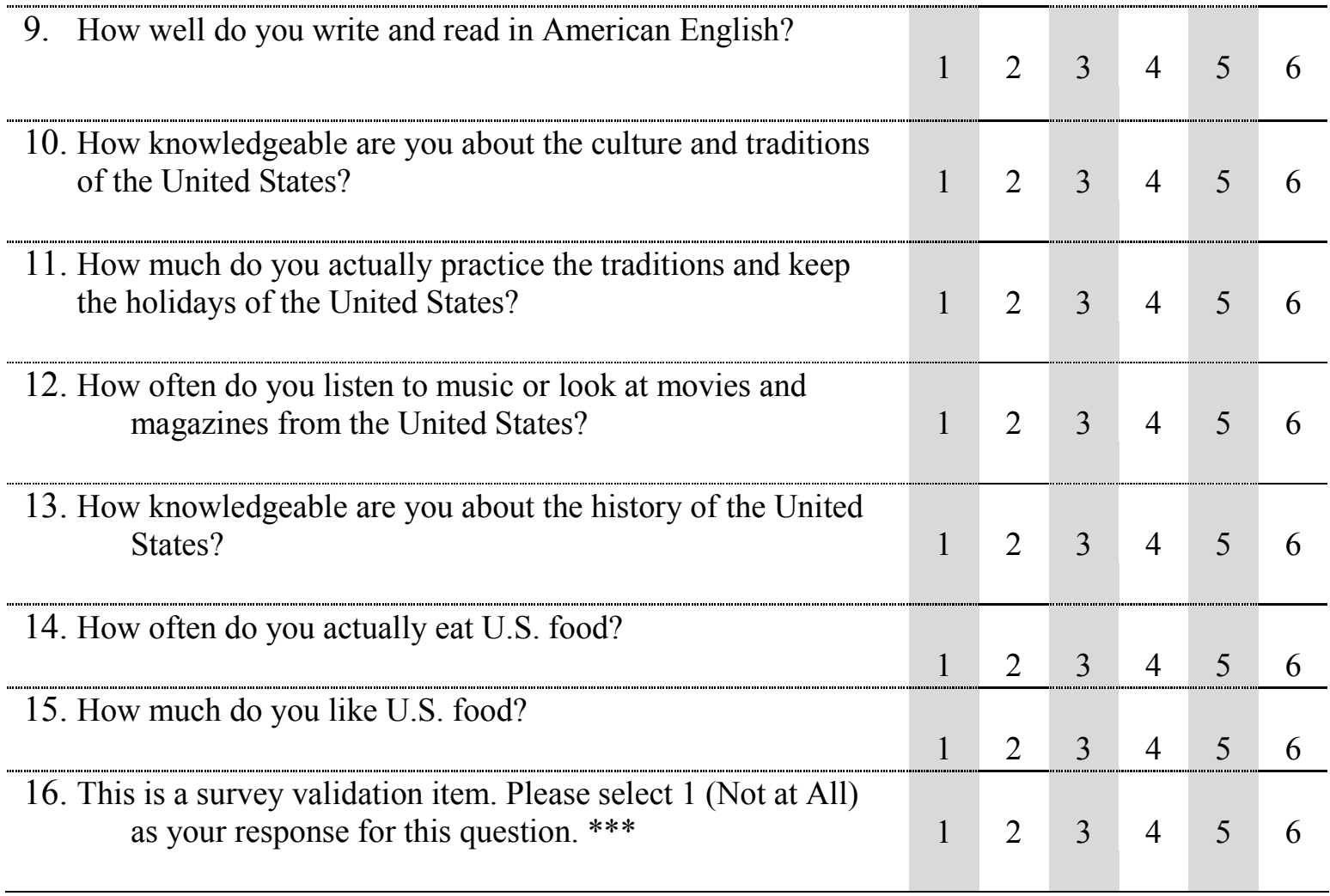

Note: Triple asterisk indicates a survey validation item. 


\section{APPENDIX K}

\section{ASIAN AMERICAN VALUES SCALE - MULTIDIMENSIONAL}

Directions: Please respond to the following items as accurately and honestly as possible. There are no wrong answers to these questions. It is important that you answer every item. Please select the number for each statement to indicate your response.

\begin{tabular}{|c|c|c|c|c|c|c|c|}
\hline & 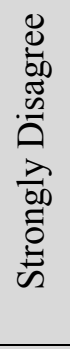 & 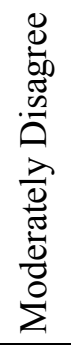 & 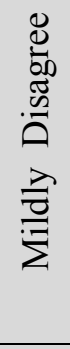 & 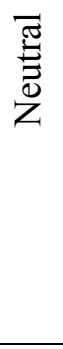 & 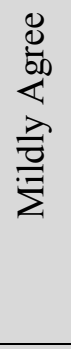 & 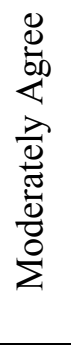 & 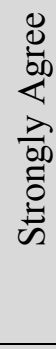 \\
\hline $\begin{array}{l}\text { 1. One should recognize and adhere to the social } \\
\text { expectation, norms, and practices. }\end{array}$ & 1 & 2 & 3 & 4 & 5 & 6 & 7 \\
\hline $\begin{array}{l}\text { 2. The welfare of the group should be put before that of } \\
\text { the individual. }\end{array}$ & 1 & 2 & 3 & 4 & 5 & 6 & 7 \\
\hline 3. It is better to show emotions than to suffer quietly. * & 1 & 2 & 3 & 4 & 5 & 6 & 7 \\
\hline $\begin{array}{l}\text { 4. One should go as far as one can academically and } \\
\text { professionally on behalf of one's family. }\end{array}$ & 1 & 2 & 3 & 4 & 5 & 6 & 7 \\
\hline $\begin{array}{l}\text { 5. One should be able to boast about one's } \\
\text { achievements. * }\end{array}$ & 1 & 2 & 3 & 4 & 5 & 6 & 7 \\
\hline $\begin{array}{l}\text { 6. One's personal needs should be second to the needs of } \\
\text { the group. }\end{array}$ & 1 & 2 & 3 & 4 & 5 & 6 & 7 \\
\hline 7. One should not express strong emotions. & 1 & 2 & 3 & 4 & 5 & 6 & 7 \\
\hline $\begin{array}{l}\text { 8. One's academic and educational occupation reflects } \\
\text { the family's reputation. }\end{array}$ & 1 & 2 & 3 & 4 & 5 & 6 & 7 \\
\hline $\begin{array}{l}\text { 9. One should be able to draw attention to one's } \\
\text { accomplishments. * }\end{array}$ & 1 & 2 & 3 & 4 & 5 & 6 & 7 \\
\hline
\end{tabular}




\begin{tabular}{|c|c|c|c|c|c|c|c|}
\hline $\begin{array}{l}\text { 10. The needs of the community should supersede those } \\
\text { of the individual. }\end{array}$ & 1 & 2 & 3 & 4 & 5 & 6 & 7 \\
\hline $\begin{array}{l}\text { 11. One should adhere to the values, beliefs, and } \\
\text { behaviors that one's society considers normal and } \\
\text { acceptable. }\end{array}$ & 1 & 2 & 3 & 4 & 5 & 6 & 7 \\
\hline $\begin{array}{l}\text { 12. Succeeding occupationally is an important way of } \\
\text { making one's family proud. }\end{array}$ & 1 & 2 & 3 & 4 & 5 & 6 & 7 \\
\hline $\begin{array}{l}\text { 13. Academic achievement should be highly valued } \\
\text { among family members. }\end{array}$ & 1 & 2 & 3 & 4 & 5 & 6 & 7 \\
\hline $\begin{array}{l}\text { 14. The group should be less important than the } \\
\text { individual. * }\end{array}$ & 1 & 2 & 3 & 4 & 5 & 6 & 7 \\
\hline $\begin{array}{l}\text { 15. One's emotional needs are less important than } \\
\text { fulfilling one's responsibilities. }\end{array}$ & 1 & 2 & 3 & 4 & 5 & 6 & 7 \\
\hline $\begin{array}{l}\text { 16. Receiving awards for excellence need not reflect well } \\
\text { on one's family. * }\end{array}$ & 1 & 2 & 3 & 4 & 5 & 6 & 7 \\
\hline $\begin{array}{l}\text { 17. One should achieve academically since it reflects on } \\
\text { one's family. }\end{array}$ & 1 & 2 & 3 & 4 & 5 & 6 & 7 \\
\hline $\begin{array}{l}\text { 18. One's educational success is a sign of personal and } \\
\text { family character. }\end{array}$ & 1 & 2 & 3 & 4 & 5 & 6 & 7 \\
\hline 19. One should not sing one's own praises. & 1 & 2 & 3 & 4 & 5 & 6 & 7 \\
\hline 20. One should not act based on emotions. & 1 & 2 & 3 & 4 & 5 & 6 & 7 \\
\hline $\begin{array}{l}\text { 21. One should work hard so that one won't be a } \\
\text { disappointment to one's family. }\end{array}$ & 1 & 2 & 3 & 4 & 5 & 6 & 7 \\
\hline $\begin{array}{l}\text { 22. Making achievements is an important way to show } \\
\text { one's appreciation to one's family. }\end{array}$ & 1 & 2 & 3 & 4 & 5 & 6 & 7 \\
\hline $\begin{array}{l}\text { 23. One's efforts should be directed toward maintaining } \\
\text { the well-being of the group first and the individual } \\
\text { second. }\end{array}$ & 1 & 2 & 3 & 4 & 5 & 6 & 7 \\
\hline $\begin{array}{l}\text { 24. It is better to hold one's emotions inside than to } \\
\text { burden others by expressing them. }\end{array}$ & 1 & 2 & 3 & 4 & 5 & 6 & 7 \\
\hline 25. One need not blend in with society. * & 1 & 2 & 3 & 4 & 5 & 6 & 7 \\
\hline
\end{tabular}




\begin{tabular}{|c|c|c|c|c|c|c|c|}
\hline $\begin{array}{l}\text { 26. Being boastful should not be a sign of one's weakness } \\
\text { and insecurity. * }\end{array}$ & 1 & 2 & 3 & 4 & 5 & 6 & 7 \\
\hline $\begin{array}{l}\text { 27. Conforming to norms provides order in the } \\
\text { community. }\end{array}$ & 1 & 2 & 3 & 4 & 5 & 6 & 7 \\
\hline 28. Conforming to norms provides one with identity. & 1 & 2 & 3 & 4 & 5 & 6 & 7 \\
\hline $\begin{array}{l}\text { 29. It is more important to behave appropriately than to } \\
\text { act on what one is feeling. }\end{array}$ & 1 & 2 & 3 & 4 & 5 & 6 & 7 \\
\hline $\begin{array}{l}\text { 30. One should not openly talk about one's } \\
\text { accomplishments. }\end{array}$ & 1 & 2 & 3 & 4 & 5 & 6 & 7 \\
\hline 31. Failing academically brings shame to one's family. & 1 & 2 & 3 & 4 & 5 & 6 & 7 \\
\hline 32. One should be expressive with one's feelings. * & 1 & 2 & 3 & 4 & 5 & 6 & 7 \\
\hline $\begin{array}{l}\text { 33. Children's achievement need not bring honor to their } \\
\text { parents. * }\end{array}$ & 1 & 2 & 3 & 4 & 5 & 6 & 7 \\
\hline $\begin{array}{l}\text { 34. One need not sacrifice oneself for the benefit of the } \\
\text { group. * }\end{array}$ & 1 & 2 & 3 & 4 & 5 & 6 & 7 \\
\hline $\begin{array}{l}\text { 35. Openly expressing one's emotions is a sign of } \\
\text { strength.* }\end{array}$ & 1 & 2 & 3 & 4 & 5 & 6 & 7 \\
\hline $\begin{array}{l}\text { 36. One's achievement and status reflects on the whole } \\
\text { family. }\end{array}$ & 1 & 2 & 3 & 4 & 5 & 6 & 7 \\
\hline $\begin{array}{l}\text { 37. One need not always consider the needs of the group } \\
\text { first.* }\end{array}$ & 1 & 2 & 3 & 4 & 5 & 6 & 7 \\
\hline $\begin{array}{l}\text { 38. It's one's duty to bring praise through achievement to } \\
\text { one's family. }\end{array}$ & 1 & 2 & 3 & 4 & 5 & 6 & 7 \\
\hline $\begin{array}{l}\text { 39. One should not do something that is outside of the } \\
\text { norm. }\end{array}$ & 1 & 2 & 3 & 4 & 5 & 6 & 7 \\
\hline $\begin{array}{l}\text { 40. Getting into a good school reflects well on one's } \\
\text { family. }\end{array}$ & 1 & 2 & 3 & 4 & 5 & 6 & 7 \\
\hline $\begin{array}{l}\text { 41. One should be able to brag about one's achievements. } \\
*\end{array}$ & 1 & 2 & 3 & 4 & 5 & 6 & 7 \\
\hline 42. Conforming to norms is the safest path to travel. & 1 & 2 & 3 & 4 & 5 & 6 & 7 \\
\hline
\end{tabular}


43. This is a survey validation item. Please select 4 (Neutral) as your response to this question. ${ }^{* * *}$

$\begin{array}{lllll}1 & 2 & 3 & 4 & 5\end{array}$

Note. Asterisk indicates a reverse-worded item. Triple asterisk indicates a survey validation item. 


\section{APPENDIX L}

\section{LOSS OF FACE QUESTIONNAIRE}

Directions: Please respond to the following items as accurately and honestly as possible. There are no wrong answers to these questions. It is important that you answer every item. Please select the number for each statement to indicate your response.

\begin{tabular}{|c|c|c|c|c|c|c|c|}
\hline & 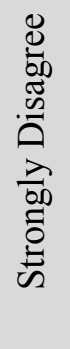 & 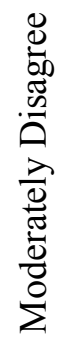 & 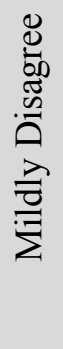 & $\underset{\overparen{Z}}{\stackrel{\pi}{0}}$ & 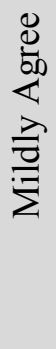 & 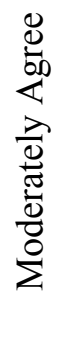 & 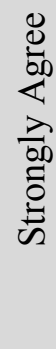 \\
\hline $\begin{array}{l}\text { 1. I am more affected when someone criticisms me in } \\
\text { public than when someone criticizes me in private. }\end{array}$ & 1 & 2 & 3 & 4 & 5 & 6 & 7 \\
\hline $\begin{array}{l}\text { 2. During a discussion, I try not to ask questions because } \\
\text { I may appear ignorant to others. }\end{array}$ & 1 & 2 & 3 & 4 & 5 & 6 & 7 \\
\hline $\begin{array}{l}\text { 3. I maintain a low profile because I do not want to make } \\
\text { mistakes in front of other people. }\end{array}$ & 1 & 2 & 3 & 4 & 5 & 6 & 7 \\
\hline $\begin{array}{l}\text { 4. Before I make a comment in the presence of other } \\
\text { people, I qualify my remarks. }\end{array}$ & 1 & 2 & 3 & 4 & 5 & 6 & 7 \\
\hline $\begin{array}{l}\text { I. I downplay my abilities and achievements so that } \\
\text { other people do not have unrealistic expectations of } \\
\text { me. }\end{array}$ & 1 & 2 & 3 & 4 & 5 & 6 & 7 \\
\hline $\begin{array}{l}\text { 6. I carefully plan what I am going to say or do to } \\
\text { minimize mistakes. }\end{array}$ & 1 & 2 & 3 & 4 & 5 & 6 & 7 \\
\hline $\begin{array}{l}\text { 7. I say I may be in error before commenting on } \\
\text { something. }\end{array}$ & 1 & 2 & 3 & 4 & 5 & 6 & 7 \\
\hline $\begin{array}{l}\text { 8. When I meet other people, I am concerned about their } \\
\text { expectations of me. }\end{array}$ & 1 & 2 & 3 & 4 & 5 & 6 & 7 \\
\hline
\end{tabular}




\begin{tabular}{|c|c|c|c|c|c|c|c|}
\hline $\begin{array}{l}\text { 9. I hesitate asking for help because I think my request } \\
\text { will be an inconvenience to others. }\end{array}$ & 1 & 2 & 3 & 4 & 5 & 6 & 7 \\
\hline 10. I try not to do things which call attention to myself. & 1 & 2 & 3 & 4 & 5 & 6 & 7 \\
\hline $\begin{array}{l}\text { 11. I do not criticize others because this may embarrass } \\
\text { them. }\end{array}$ & 1 & 2 & 3 & 4 & 5 & 6 & 7 \\
\hline 12. I carefully watch others' actions before I do anything. & 1 & 2 & 3 & 4 & 5 & 6 & 7 \\
\hline $\begin{array}{l}\text { 13. I will not complain publicly even when I have been } \\
\text { treated unfairly. }\end{array}$ & 1 & 2 & 3 & 4 & 5 & 6 & 7 \\
\hline $\begin{array}{l}\text { 14. I try to act like others to be consistent with social } \\
\text { norms. }\end{array}$ & 1 & 2 & 3 & 4 & 5 & 6 & 7 \\
\hline $\begin{array}{l}\text { 15. Before I do anything in public, I prepare myself for } \\
\text { any possible consequence. }\end{array}$ & 1 & 2 & 3 & 4 & 5 & 6 & 7 \\
\hline $\begin{array}{l}\text { 16. I prefer to use a third party to help resolve our } \\
\text { differences between another person and me. }\end{array}$ & 1 & 2 & 3 & 4 & 5 & 6 & 7 \\
\hline $\begin{array}{l}\text { 17. When discussing a problem, I make an effort to let the } \\
\text { person know that I am not blaming him or her. }\end{array}$ & 1 & 2 & 3 & 4 & 5 & 6 & 7 \\
\hline $\begin{array}{l}\text { 18. When someone criticizes me, I try to avoid that } \\
\text { person. }\end{array}$ & 1 & 2 & 3 & 4 & 5 & 6 & 7 \\
\hline $\begin{array}{l}\text { 19. When I make a mistake in front of others, I try to } \\
\text { prevent them from noticing it. }\end{array}$ & 1 & 2 & 3 & 4 & 5 & 6 & 7 \\
\hline $\begin{array}{l}\text { 20. Even when I know another person is at fault, I am } \\
\text { careful to not criticize that person. }\end{array}$ & 1 & 2 & 3 & 4 & 5 & 6 & 7 \\
\hline 21. When someone embarrasses me, I try to forget it. & 1 & 2 & 3 & 4 & 5 & 6 & 7 \\
\hline $\begin{array}{l}\text { 22. This is a survey validation item. Please select } 7 \\
\text { (Strongly Agree) as your response to this question. } \\
* * *\end{array}$ & 1 & 2 & 3 & 4 & 5 & 6 & 7 \\
\hline
\end{tabular}

Note: Triple asterisk indicates a survey validation item. 